\title{
Pd-Catalyzed Dearomative Asymmetric Allylic Alkylation of Naphthols with Alkoxyallenes
}

Jinjin $\mathrm{Hu},{ }^{\dagger}$ Shulei Pan, ${ }^{\dagger}$ Shuai Zhu,${ }^{\ddagger}$ Peiyuan $\mathrm{Yu},{ }^{\ddagger}$ Ruigang $\mathrm{Xu},{ }^{\dagger}$ Guofu Zhong,${ }^{\dagger}$ Xiaofei Zeng*,

${ }^{\dagger}$ College of Material Chemistry and Chemical Engineering, Hangzhou Normal University, Hangzhou 310036, China.

\$Shenzhen Grubbs Institute and Department of Chemistry, Southern University of Science and Technology, Shenzhen 518055, China.

Email: chemzxf@hznu.edu.cn

\section{Table of Content}

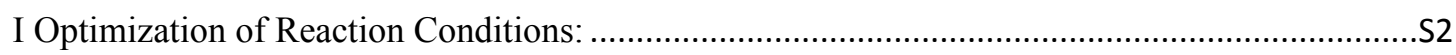

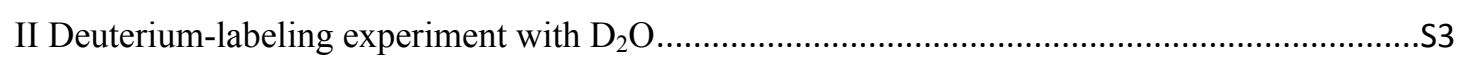

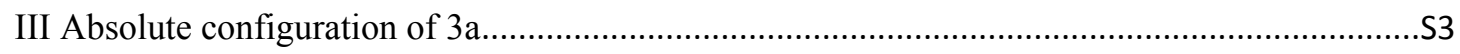

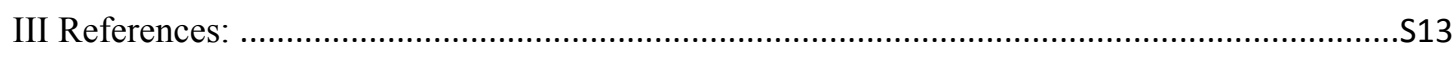

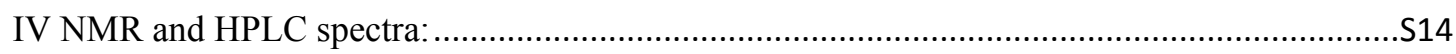




\section{Optimization of Reaction Conditions:}

Table S1. Reaction Condition Optimization ${ }^{a}$
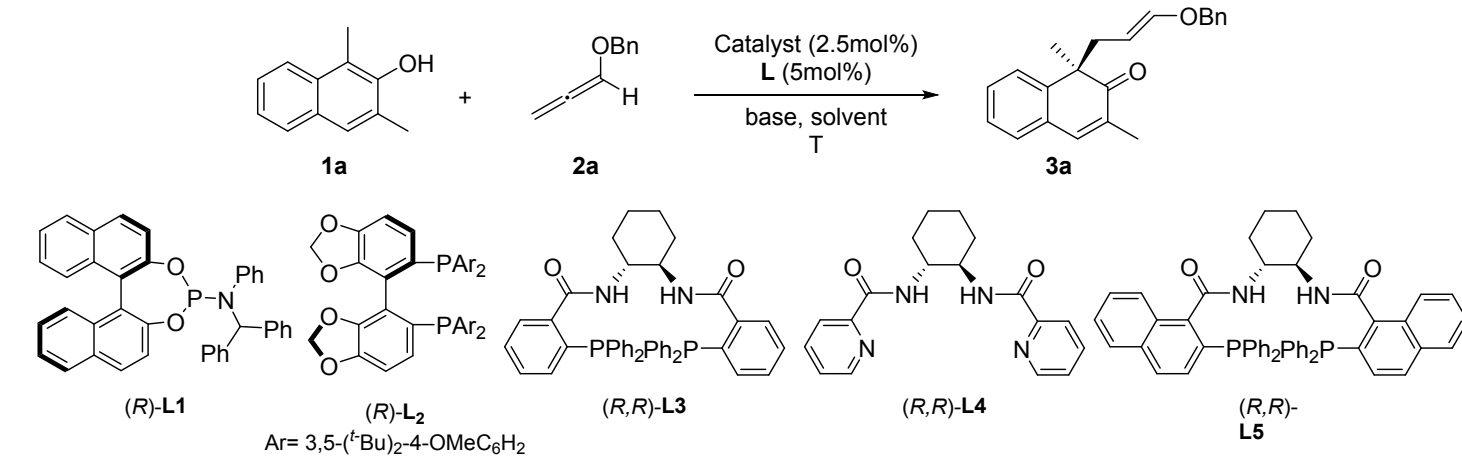

\begin{tabular}{|c|c|c|c|c|c|c|c|}
\hline entry & $\mathbf{L}$ & catalyst & base & solvent & $\mathrm{T}\left[{ }^{\circ} \mathrm{C}\right]$ & yield $[\%]^{\mathrm{b}}$ & $e e[\%]^{\mathrm{c}}$ \\
\hline 1 & $(R)-\mathbf{L} \mathbf{1}$ & $\operatorname{Pd}(\mathrm{dba})_{2}$ & $\mathrm{DBU}$ & DCE & 60 & 64 & 10 \\
\hline 2 & $(R)-\mathbf{L} 2$ & $\operatorname{Pd}(\mathrm{dba})_{2}$ & DBU & DCE & 60 & 36 & 12 \\
\hline 3 & $(R, R)-\mathbf{L} \mathbf{3}$ & $\operatorname{Pd}(\mathrm{dba})_{2}$ & $\mathrm{DBU}$ & DCE & 60 & 58 & 56 \\
\hline 4 & $(R, R)-\mathbf{L} \mathbf{3}$ & $\operatorname{Pd}(\mathrm{dba})_{2}$ & - & DCE & 60 & 25 & 49 \\
\hline 5 & $(R, R)-\mathbf{L} \mathbf{4}$ & $\operatorname{Pd}(\mathrm{dba})_{2}$ & DBU & $\mathrm{DCE}$ & 60 & - & - \\
\hline 6 & $(R, R)-\mathbf{L} \mathbf{5}$ & $\operatorname{Pd}(\mathrm{dba})_{2}$ & DBU & DCE & 60 & 63 & 64 \\
\hline 7 & $(R, R)-\mathbf{L 5}$ & $\mathrm{Pd}_{2}(\mathrm{dba})_{3}$ & DBU & DCE & 60 & 66 & 78 \\
\hline 8 & $(R, R)-\mathbf{L 5}$ & $\mathrm{Pd}_{2}(\mathrm{dba})_{3} \cdot \mathrm{CHCl}_{3}$ & $\mathrm{DBU}$ & DCE & 60 & 49 & 65 \\
\hline 9 & $(R, R)-\mathbf{L 5}$ & $\mathrm{Pd}(\mathrm{OAc})_{2}$ & $\mathrm{DBU}$ & $\mathrm{DCE}$ & 60 & 61 & 54 \\
\hline 10 & $(R, R)-\mathbf{L} \mathbf{5}$ & $\mathrm{Cu}(\mathrm{OTf})_{2}$ & $\mathrm{DBU}$ & DCE & 60 & - & - \\
\hline 11 & $(R, R)-\mathbf{L 5}$ & $\mathrm{Pd}_{2}(\mathrm{dba})_{3}$ & DBU & THF & 60 & 25 & 82 \\
\hline 12 & $(R, R)-\mathbf{L 5}$ & $\mathrm{Pd}_{2}(\mathrm{dba})_{3}$ & DBU & EA & 60 & 85 & 79 \\
\hline 13 & $(R, R)-\mathbf{L 5}$ & $\mathrm{Pd}_{2}(\mathrm{dba})_{3}$ & DBU & $\mathrm{DCM}$ & 60 & 66 & 35 \\
\hline 14 & $(R, R)-\mathbf{L 5}$ & $\mathrm{Pd}_{2}(\mathrm{dba})_{3}$ & DBU & Tol & 60 & 45 & 31 \\
\hline 15 & $(R, R)-\mathbf{L 5}$ & $\mathrm{Pd}_{2}(\mathrm{dba})_{3}$ & $\mathrm{DBU}$ & $\mathrm{MeOH}$ & 60 & - & - \\
\hline 16 & $(R, R)-\mathbf{L 5}$ & $\mathrm{Pd}_{2}(\mathrm{dba})_{3}$ & $\mathrm{Et}_{3} \mathrm{~N}$ & THF & 60 & 35 & 20 \\
\hline 17 & $(R, R)-\mathbf{L 5}$ & $\mathrm{Pd}_{2}(\mathrm{dba})_{3}$ & $\mathrm{DABCO}$ & THF & 60 & 55 & 0 \\
\hline 18 & $(R, R)-\mathbf{L 5}$ & $\mathrm{Pd}_{2}(\mathrm{dba})_{3}$ & pridine & THF & 60 & 48 & 0 \\
\hline 19 & $(R, R)-\mathbf{L 5}$ & $\mathrm{Pd}_{2}(\mathrm{dba})_{3}$ & $\mathrm{Na}_{2} \mathrm{CO}_{3}$ & THF & 60 & 50 & 45 \\
\hline 20 & $(R, R)-\mathbf{L 5}$ & $\mathrm{Pd}_{2}(\mathrm{dba})_{3}$ & TMG & $\mathrm{THF}$ & 60 & 34 & 83 \\
\hline 21 & $(R, R)-\mathbf{L 5}$ & $\mathrm{Pd}_{2}(\mathrm{dba})_{3}$ & TMG & MTBE & 60 & 68 & 81 \\
\hline 22 & $(R, R)-\mathbf{L 5}$ & $P d_{2}(d b a)_{3}$ & TMG & PO & 60 & 81 & 84 \\
\hline 23 & $(R, R)-\mathbf{L 5}$ & $\mathrm{Pd}_{2}(\mathrm{dba})_{3}$ & $\mathrm{TMG}$ & $\mathrm{PO}$ & 25 & - & - \\
\hline $24^{\mathrm{d}}$ & $(R, R)-\mathbf{L 5}$ & $\mathrm{Pd}_{2}(\mathrm{dba})_{3}$ & $\mathrm{TMG}$ & $\mathrm{PO}$ & 60 & 56 & 65 \\
\hline $25^{\mathrm{e}}$ & $(R, R)-\mathbf{L 5}$ & $\mathrm{Pd}_{2}(\mathrm{dba})_{3}$ & TMG & $\mathrm{PO}$ & 60 & 30 & 81 \\
\hline $26^{\mathrm{f}}$ & $(R, R)-\mathbf{L 5}$ & $\mathrm{Pd}_{2}(\mathrm{dba})_{3}$ & TMG & $\mathrm{PO}$ & 60 & 63 & 21 \\
\hline
\end{tabular}

${ }^{\mathrm{a}}$ General conditions: 1a $(0.2 \mathrm{mmol}), \mathbf{2 a}(0.3 \mathrm{mmol}),\left[\mathrm{Pd}_{2}(\mathrm{dba})_{3}(2.5 \mathrm{~mol} \%)\right.$, ligand $(5 \mathrm{~mol} \%)$, and base $(02$ $\mathrm{mmol}$ ) in $2 \mathrm{~mL}$ solvent under argon at $60{ }^{\circ} \mathrm{C}$. b Isolated yields after column chromatography. ${ }^{\mathrm{c}}$ Determined by HPLC on a chiral stationary phase. ${ }^{\mathrm{d}} 3 \AA \mathrm{MS}$ (30 mg) was added. ${ }^{\mathrm{e}} 5 \AA \mathrm{MS}$ (30 mg) was added. ${ }^{\mathrm{f}} 0.3 \mathrm{mmol}$ base was used. 


\section{Deuterium-labeling experiment with $\mathrm{D}_{2} \mathrm{O}$}

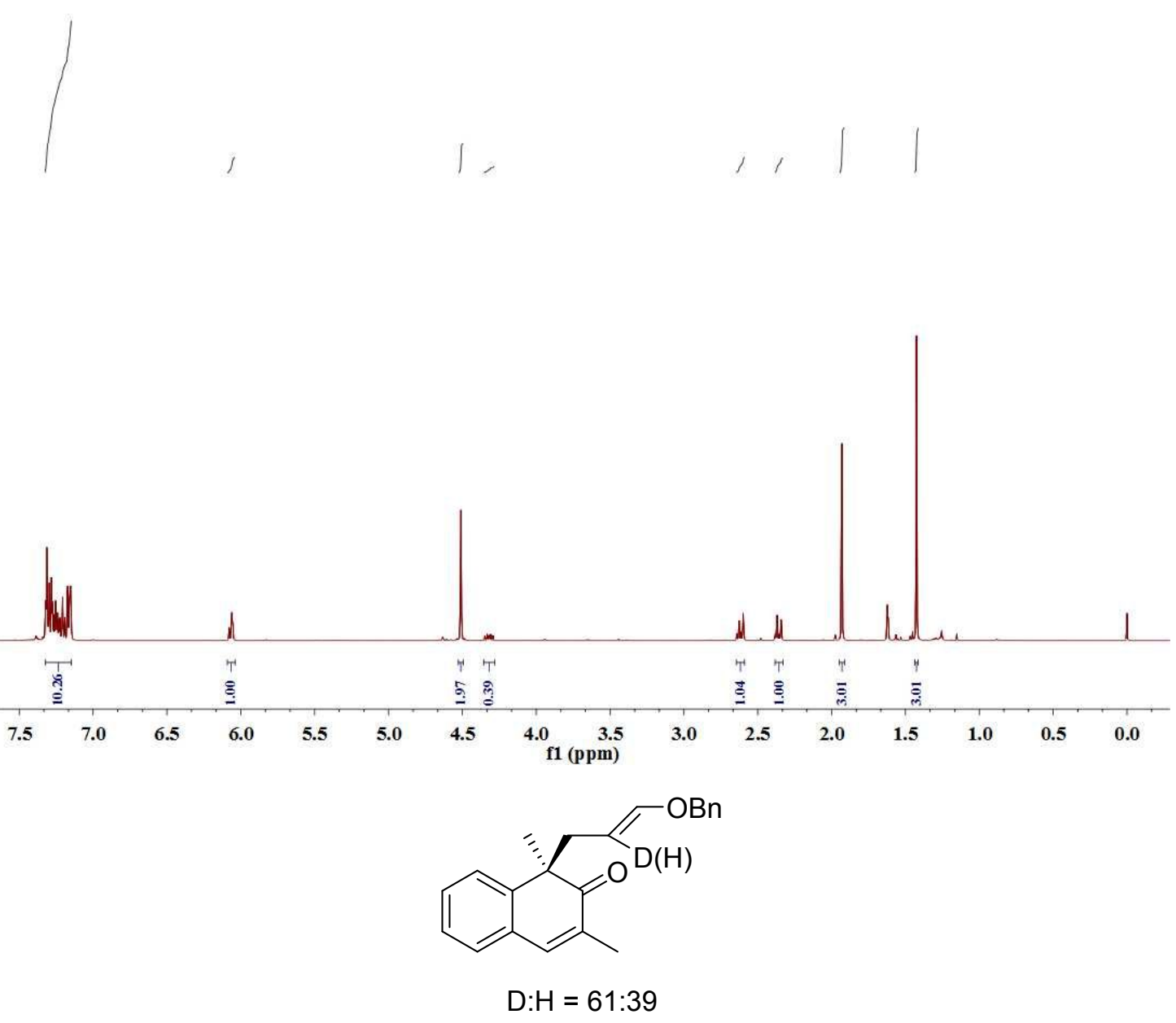

\section{Absolute configuration of 3a}

\section{Computational details}

To simulate the CD spectra of compound 3a, extensive conformational searches were first performed with Micromodel ${ }^{1}$ using the MMFF force field. A mixed torsional/low-mode sampling method was used to generate conformers within a $5 \mathrm{kcal} / \mathrm{mol}$ energy window. Subsequent density functional theory calculations were performed with the Gaussian 16 package. ${ }^{2}$ Geometry optimizations were performed with B3LYP functional ${ }^{3,4}$ and the $6-311+\mathrm{G}(\mathrm{d}, \mathrm{p})$ basis set in the gas phase initially. The lowest energy conformers within $2 \mathrm{kcal} / \mathrm{mol}$ were then re-optimized with the SMD solvation model ${ }^{5}$ in cyclohexane. These optimized structures were verified by normal mode vibrational analysis as minima (zero imaginary frequency). Free energies were corrected using Truhlar's quasiharmonic correction, by raising vibrational frequencies that are below $100 \mathrm{~cm}^{-1}$ to $100 \mathrm{~cm}^{-1} .6$ Time-dependent density functional theory (TD-DFT) single point calculations were computed at the same level of theory with 20 excited states. Only the lowest energy conformer of within $1 \mathrm{kcal} / \mathrm{mol}$ used for calculating the $\mathrm{CD}$ spectra. The final results are the weighted average of all these different conformations. The simulated spectra are blue- 
shifted by $25 \mathrm{~nm}$ to better compare with the experimental spectra. Finally, the $R$ configuration could be reliably assigned to compound $\mathbf{3 a}$.

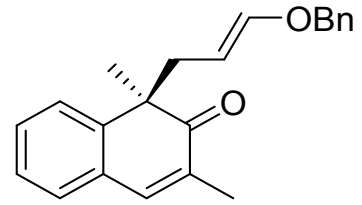

3a

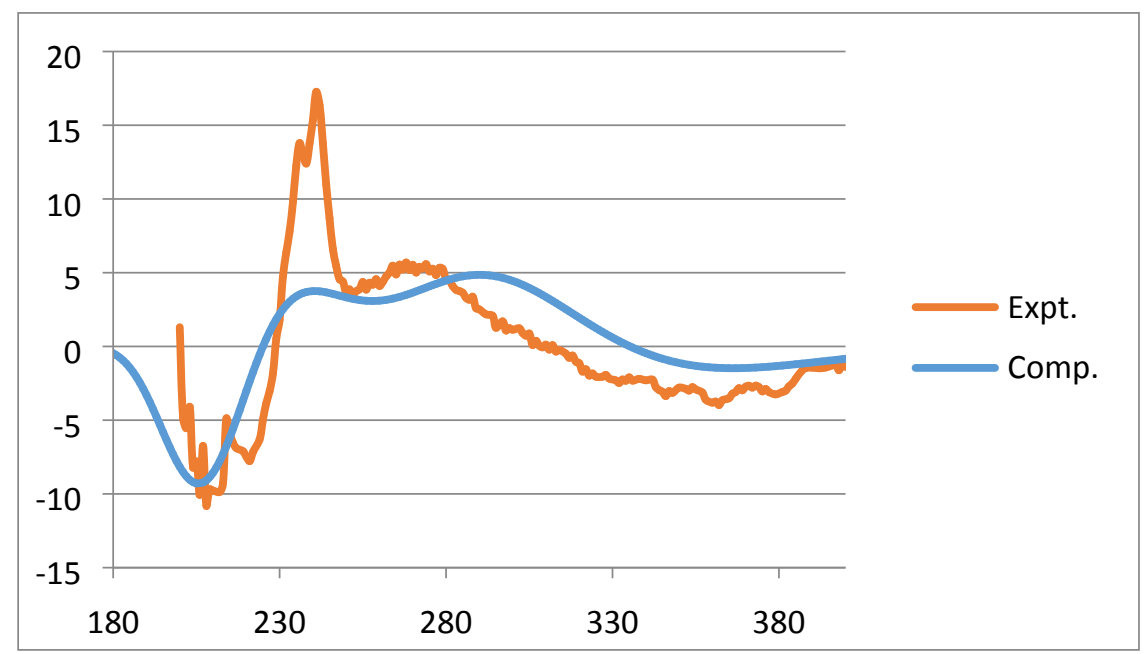

Figure S1. Experimental and Computed CD Spectra of 3a

Free energies and percentage composition for the different conformations of compound $\mathbf{3 a}$ within 1 $\mathrm{kcal} / \mathrm{mol}$ :

$\begin{array}{ccccc}\text { Conformation } & G \text { (Hartree) } & \Delta G(\mathrm{kcal} / \mathrm{mol}) & \text { Ratio } & \text { Percentage } \\ \text { X_3 } & -1001.965738 & 0.00 & 1.00 & 29.8 \% \\ \text { X_7 } & -1001.965346 & 0.25 & 0.66 & 19.6 \% \\ \text { X_13 } & -1001.965098 & 0.40 & 0.51 & 15.1 \% \\ \text { X_15 } & -1001.965017 & 0.45 & 0.47 & 13.9 \% \\ \text { X_22 } & -1001.964632 & 0.69 & 0.31 & 9.2 \% \\ \text { X_26 } & -1001.964314 & 0.89 & 0.22 & 6.6 \% \\ \text { X_19 } & -1001.964195 & 0.97 & 0.20 & 5.8 \%\end{array}$

Cartesian coordinates of DFT-computed structures:

$\begin{array}{llll}\text { X_3 } & & & \\ \text { C } & -3.02196 & 1.95390 & -0.95990 \\ \text { C } & -3.15618 & 3.19088 & -0.33203 \\ \text { C } & -2.77647 & 0.78859 & -0.22797 \\ \text { C } & -3.04896 & 3.28686 & 1.05556 \\ \text { C } & -2.80767 & 2.14016 & 1.79938 \\ \text { C } & -2.66922 & 0.88933 & 1.17332 \\ \text { H } & -3.11163 & 1.90376 & -2.03904 \\ \text { H } & -3.34619 & 4.07760 & -0.92653 \\ \text { H } & -3.15438 & 4.24631 & 1.54892\end{array}$




\begin{tabular}{|c|c|c|c|}
\hline $\mathrm{H}$ & -2.72376 & 2.19911 & 2.87981 \\
\hline $\mathrm{C}$ & -2.64270 & -0.55982 & -0.92861 \\
\hline $\mathrm{C}$ & -2.35699 & -1.74843 & 0.01665 \\
\hline $\mathrm{C}$ & -2.26615 & -1.54094 & 1.47372 \\
\hline $\mathrm{C}$ & -2.41691 & -0.29576 & 1.97516 \\
\hline $\mathrm{C}$ & -2.00129 & -2.74583 & 2.32890 \\
\hline $\mathrm{H}$ & -2.34784 & -0.14769 & 3.05042 \\
\hline $\mathrm{H}$ & -1.05880 & -3.22517 & 2.04795 \\
\hline $\mathrm{H}$ & -2.78218 & -3.50060 & 2.19618 \\
\hline $\mathrm{H}$ & -1.95415 & -2.47343 & 3.38529 \\
\hline $\mathrm{C}$ & -3.97061 & -0.89580 & -1.65778 \\
\hline $\mathrm{H}$ & -3.88998 & -1.87191 & -2.13942 \\
\hline $\mathrm{H}$ & -4.19264 & -0.14555 & -2.41971 \\
\hline $\mathrm{H}$ & -4.80922 & -0.92368 & -0.95672 \\
\hline $\mathrm{C}$ & -1.48866 & -0.52719 & -1.98880 \\
\hline $\mathrm{C}$ & -0.12667 & -0.18958 & -1.44570 \\
\hline $\mathrm{H}$ & -1.77239 & 0.19856 & -2.75886 \\
\hline $\mathrm{H}$ & -1.46779 & -1.50849 & -2.46804 \\
\hline $\mathrm{C}$ & 0.89011 & -1.05767 & -1.49266 \\
\hline $\mathrm{H}$ & 0.00581 & 0.79756 & -1.01718 \\
\hline $\mathrm{O}$ & 2.16720 & -0.88890 & -1.06050 \\
\hline $\mathrm{H}$ & 0.77474 & -2.05062 & -1.91579 \\
\hline $\mathrm{C}$ & 2.50268 & 0.37380 & -0.46018 \\
\hline $\mathrm{C}$ & 3.95509 & 0.34863 & -0.06828 \\
\hline $\mathrm{H}$ & 1.86364 & 0.53470 & 0.41531 \\
\hline $\mathrm{H}$ & 2.30759 & 1.17632 & -1.18033 \\
\hline $\mathrm{C}$ & 4.34436 & -0.13613 & 1.18412 \\
\hline $\mathrm{C}$ & 4.93863 & 0.78878 & -0.95906 \\
\hline $\mathrm{C}$ & 6.28622 & 0.74534 & -0.60656 \\
\hline $\mathrm{C}$ & 6.66444 & 0.25957 & 0.64445 \\
\hline $\mathrm{C}$ & 5.69076 & -0.18157 & 1.54002 \\
\hline $\mathrm{H}$ & 3.58848 & -0.48106 & 1.88227 \\
\hline $\mathrm{H}$ & 4.64657 & 1.16624 & -1.93376 \\
\hline $\mathrm{H}$ & 7.03923 & 1.09110 & -1.30610 \\
\hline $\mathrm{H}$ & 7.71247 & 0.22688 & 0.92102 \\
\hline $\mathrm{H}$ & 5.97983 & -0.55906 & 2.51464 \\
\hline $\mathrm{O}$ & -2.22203 & -2.86779 & -0.45985 \\
\hline \multicolumn{4}{|c|}{ X_7 } \\
\hline $\mathrm{C}$ & -2.62185 & 1.89898 & -1.20949 \\
\hline $\mathrm{C}$ & -2.55943 & 3.20951 & -0.74017 \\
\hline $\mathrm{C}$ & -2.59820 & 0.80992 & -0.33426 \\
\hline $\mathrm{C}$ & -2.47413 & 3.45995 & 0.62947 \\
\hline $\mathrm{C}$ & -2.45412 & 2.39160 & 1.51476 \\
\hline
\end{tabular}




\begin{tabular}{|c|c|c|c|}
\hline $\mathrm{C}$ & -2.51570 & 1.06707 & 1.04834 \\
\hline $\mathrm{H}$ & -2.69036 & 1.73112 & -2.27821 \\
\hline $\mathrm{H}$ & -2.57758 & 4.03378 & -1.44452 \\
\hline $\mathrm{H}$ & -2.42529 & 4.47777 & 0.99921 \\
\hline $\mathrm{H}$ & -2.39198 & 2.57001 & 2.58349 \\
\hline $\mathrm{C}$ & -2.68158 & -0.61816 & -0.86130 \\
\hline $\mathrm{C}$ & -2.63211 & -1.70970 & 0.23195 \\
\hline $\mathrm{C}$ & -2.55994 & -1.33880 & 1.65628 \\
\hline $\mathrm{C}$ & -2.50168 & -0.03236 & 1.99675 \\
\hline $\mathrm{C}$ & -2.54637 & -2.45267 & 2.66322 \\
\hline $\mathrm{H}$ & -2.44194 & 0.23556 & 3.04920 \\
\hline $\mathrm{H}$ & -1.69578 & -3.12113 & 2.49954 \\
\hline $\mathrm{H}$ & -3.44430 & -3.07164 & 2.57550 \\
\hline $\mathrm{H}$ & -2.49108 & -2.05970 & 3.68050 \\
\hline $\mathrm{C}$ & -4.03125 & -0.81592 & -1.60241 \\
\hline $\mathrm{H}$ & -4.87765 & -0.61503 & -0.94010 \\
\hline $\mathrm{H}$ & -4.10811 & -1.84416 & -1.96039 \\
\hline $\mathrm{H}$ & -4.10453 & -0.13995 & -2.45698 \\
\hline $\mathrm{C}$ & -1.51656 & -0.91824 & -1.86856 \\
\hline $\mathrm{C}$ & -0.12986 & -0.83919 & -1.29220 \\
\hline $\mathrm{H}$ & -1.60848 & -0.23369 & -2.71589 \\
\hline $\mathrm{H}$ & -1.69999 & -1.92721 & -2.25011 \\
\hline $\mathrm{C}$ & 0.77293 & 0.05202 & -1.71472 \\
\hline $\mathrm{H}$ & 0.12341 & -1.55690 & -0.51940 \\
\hline $\mathrm{O}$ & 2.05623 & 0.22071 & -1.30073 \\
\hline $\mathrm{H}$ & 0.54307 & 0.77347 & -2.49288 \\
\hline $\mathrm{C}$ & 2.53396 & -0.64575 & -0.25720 \\
\hline $\mathrm{C}$ & 3.96249 & -0.28682 & 0.04969 \\
\hline $\mathrm{H}$ & 2.45736 & -1.68652 & -0.59088 \\
\hline $\mathrm{H}$ & 1.90006 & -0.52018 & 0.62789 \\
\hline $\mathrm{C}$ & 5.01502 & -0.95876 & -0.57858 \\
\hline $\mathrm{C}$ & 4.25760 & 0.73837 & 0.95391 \\
\hline $\mathrm{C}$ & 5.57912 & 1.08682 & 1.22428 \\
\hline $\mathrm{C}$ & 6.62250 & 0.41026 & 0.59265 \\
\hline $\mathrm{C}$ & 6.33846 & -0.61385 & -0.30959 \\
\hline $\mathrm{H}$ & 4.79620 & -1.75573 & -1.28195 \\
\hline $\mathrm{H}$ & 3.44727 & 1.26592 & 1.44665 \\
\hline $\mathrm{H}$ & 5.79500 & 1.88288 & 1.92832 \\
\hline $\mathrm{H}$ & 7.65165 & 0.67832 & 0.80467 \\
\hline $\mathrm{H}$ & 7.14597 & -1.14413 & -0.80216 \\
\hline $\mathrm{O}$ & -2.66356 & -2.88805 & -0.10157 \\
\hline \multicolumn{4}{|c|}{ X_13 } \\
\hline $\mathrm{C}$ & -3.47644 & 1.75772 & -0.96027 \\
\hline
\end{tabular}




\begin{tabular}{|c|c|c|c|}
\hline $\mathrm{C}$ & -3.84676 & 2.94917 & -0.33943 \\
\hline $\mathrm{C}$ & -3.03224 & 0.65875 & -0.21986 \\
\hline $\mathrm{C}$ & -3.78207 & 3.06454 & 1.04926 \\
\hline $\mathrm{C}$ & -3.34527 & 1.98300 & 1.80133 \\
\hline $\mathrm{C}$ & -2.96810 & 0.77894 & 1.18228 \\
\hline $\mathrm{H}$ & -3.53546 & 1.69145 & -2.04062 \\
\hline $\mathrm{H}$ & -4.18537 & 3.78572 & -0.94057 \\
\hline $\mathrm{H}$ & -4.06896 & 3.98914 & 1.53702 \\
\hline $\mathrm{H}$ & -3.28902 & 2.05802 & 2.88249 \\
\hline $\mathrm{C}$ & -2.64183 & -0.64286 & -0.91232 \\
\hline $\mathrm{C}$ & -2.15081 & -1.75519 & 0.04116 \\
\hline $\mathrm{C}$ & -2.12460 & -1.53344 & 1.49770 \\
\hline $\mathrm{C}$ & -2.51177 & -0.33724 & 1.99177 \\
\hline $\mathrm{C}$ & -1.65133 & -2.66549 & 2.36267 \\
\hline $\mathrm{H}$ & -2.48515 & -0.17678 & 3.06700 \\
\hline $\mathrm{H}$ & -0.62805 & -2.95373 & 2.10394 \\
\hline $\mathrm{H}$ & -2.26927 & -3.55651 & 2.21674 \\
\hline $\mathrm{H}$ & -1.67954 & -2.38911 & 3.41868 \\
\hline $\mathrm{C}$ & -3.87629 & -1.22126 & -1.65359 \\
\hline $\mathrm{H}$ & -4.70336 & -1.40100 & -0.96142 \\
\hline $\mathrm{H}$ & -3.61148 & -2.16728 & -2.12939 \\
\hline $\mathrm{H}$ & -4.22403 & -0.52680 & -2.42149 \\
\hline $\mathrm{C}$ & -1.50298 & -0.39879 & -1.96218 \\
\hline $\mathrm{C}$ & -0.23009 & 0.17342 & -1.40711 \\
\hline $\mathrm{H}$ & -1.90662 & 0.26847 & -2.73149 \\
\hline $\mathrm{H}$ & -1.30327 & -1.35894 & -2.44338 \\
\hline $\mathrm{C}$ & 0.92171 & -0.49877 & -1.39989 \\
\hline $\mathrm{H}$ & -0.25202 & 1.17448 & -0.98555 \\
\hline $\mathrm{O}$ & 2.06141 & 0.01129 & -0.85071 \\
\hline $\mathrm{H}$ & 1.00664 & -1.50468 & -1.80856 \\
\hline $\mathrm{C}$ & 3.27353 & -0.67805 & -1.21383 \\
\hline $\mathrm{C}$ & 4.41528 & -0.11754 & -0.41060 \\
\hline $\mathrm{H}$ & 3.45597 & -0.54679 & -2.28634 \\
\hline $\mathrm{H}$ & 3.14884 & -1.74876 & -1.01317 \\
\hline $\mathrm{C}$ & 4.64664 & -0.56061 & 0.89594 \\
\hline $\mathrm{C}$ & 5.24840 & 0.86693 & -0.94871 \\
\hline $\mathrm{C}$ & 6.29548 & 1.39935 & -0.19772 \\
\hline $\mathrm{C}$ & 6.51763 & 0.95180 & 1.10336 \\
\hline $\mathrm{C}$ & 5.69035 & -0.02965 & 1.64990 \\
\hline $\mathrm{H}$ & 4.00474 & -1.32435 & 1.32296 \\
\hline $\mathrm{H}$ & 5.07574 & 1.21856 & -1.96079 \\
\hline $\mathrm{H}$ & 6.93605 & 2.16134 & -0.62773 \\
\hline $\mathrm{H}$ & 7.33251 & 1.36351 & 1.68850 \\
\hline $\mathrm{H}$ & 5.86069 & -0.38255 & 2.66103 \\
\hline
\end{tabular}


X_15

C

$$
-3.37857
$$

1.75534

$-1.13517$

C $\quad-3.74310$

2.98796

$-0.59688$

C

$-3.01571$

0.68242

$-0.31653$

C $\quad-3.75451$

3.17207

0.78585

C $\quad-3.39945$

2.11716

1.61487

$\begin{array}{ll}\text { C } & -3.02889\end{array}$

0.87170

1.07938

$\mathrm{H}$

1.63543

$-2.21232$

$\mathrm{H}$

$-3.37638$

3.80300

$-1.25744$

$\mathrm{H} \quad-4.03691$

4.12906

1.20952

$\mathrm{H}$

$-3.40306$

2.24612

2.69231

C $\quad-2.63196$

$-0.66445$

$-0.92044$

C $\quad-2.22282$

$-1.73781$

0.11302

C $\quad-2.28078$

$-1.44574$

1.55645

C $\quad-2.65972$

$-0.21608$

1.96817

$-2.54747$

2.50188

$\mathrm{H} \quad-2.69479$

$-0.00430$

3.03440

$\mathrm{H} \quad-0.87203$

$-2.88042$

2.32015

$\mathrm{H} \quad-2.53633$

$-3.42508$

2.36118

$-2.21832$

3.53981

C $\quad-3.84880$

$-1.24703$

$-1.68798$

$\mathrm{H} \quad-4.71196$

$-1.36540$

$-1.02737$

$\mathrm{H}$

$-3.59171$

$-2.22459$

$-2.10014$

H $\quad-4.13899$

$-0.58567$

$-2.50729$

C

$-1.43964$

$-0.50963$

$-1.92519$

C $\quad-0.17910$

0.05754

$-1.33739$

$\mathrm{H} \quad-1.78598$

0.12444

$-2.74889$

$-1.24499$

$-1.49912$

$-2.34494$

C $\quad 0.96543$

$-0.62480$

$-1.28544$

1.07274

$-0.95085$

$\mathrm{H}$

$-0.19840$

$-0.07927$

$-0.80196$

2.11856

$-1.64807$

$-1.64933$

3.17747

$-1.02317$

$-0.54607$

C $\quad 4.40750$

$-0.27150$

$-0.11632$

$-1.59615$

$-1.45977$

$-1.72009$

0.23647

2.85736

0.22917

$-1.06762$

$-0.04209$

1.23884

4.66237

0.67363

1.63785

C $\quad 5.78977$

1.16893

0.68185

C -6.67542

0.94568

$-0.67276$

$\mathrm{H} \quad 5.11258$

0.05690

$-2.12228$ 


\begin{tabular}{|c|c|c|c|}
\hline $\mathrm{H}$ & 3.97330 & -0.42584 & 1.98426 \\
\hline $\mathrm{H}$ & 5.97720 & 0.84333 & 2.69237 \\
\hline $\mathrm{H}$ & 7.55434 & 1.72406 & 0.99053 \\
\hline $\mathrm{H}$ & 7.11683 & 1.32718 & -1.41985 \\
\hline $\mathrm{O}$ & -1.86761 & -2.84075 & -0.28337 \\
\hline \multicolumn{4}{|c|}{ X_19 } \\
\hline $\mathrm{C}$ & 1.35206 & 1.71615 & 0.93055 \\
\hline C & 1.38386 & 3.08282 & 0.65104 \\
\hline $\mathrm{C}$ & 2.30319 & 0.84215 & 0.39697 \\
\hline $\mathrm{C}$ & 2.37365 & 3.60703 & -0.17836 \\
\hline $\mathrm{C}$ & 3.33695 & 2.75687 & -0.70600 \\
\hline $\mathrm{C}$ & 3.31999 & 1.38215 & -0.42029 \\
\hline $\mathrm{H}$ & 0.57338 & 1.33412 & 1.57769 \\
\hline $\mathrm{H}$ & 0.63481 & 3.73617 & 1.08477 \\
\hline $\mathrm{H}$ & 2.39935 & 4.66766 & -0.40092 \\
\hline $\mathrm{H}$ & 4.12621 & 3.15245 & -1.33716 \\
\hline $\mathrm{C}$ & 2.23410 & -0.66066 & 0.63648 \\
\hline $\mathrm{C}$ & 3.56308 & -1.39013 & 0.33953 \\
\hline $\mathrm{C}$ & 4.53914 & -0.76803 & -0.57952 \\
\hline $\mathrm{C}$ & 4.38310 & 0.52856 & -0.92293 \\
\hline $\mathrm{C}$ & 5.69318 & -1.61017 & -1.04082 \\
\hline $\mathrm{H}$ & 5.11466 & 0.99486 & -1.57870 \\
\hline $\mathrm{H}$ & 6.29795 & -1.94415 & -0.19254 \\
\hline $\mathrm{H}$ & 6.33400 & -1.05256 & -1.72701 \\
\hline $\mathrm{H}$ & 5.34301 & -2.51609 & -1.54501 \\
\hline $\mathrm{C}$ & 1.77352 & -1.01290 & 2.06026 \\
\hline $\mathrm{H}$ & 2.41106 & -0.53133 & 2.80646 \\
\hline $\mathrm{H}$ & 1.83179 & -2.09111 & 2.21025 \\
\hline $\mathrm{H}$ & 0.74313 & -0.70092 & 2.23360 \\
\hline $\mathrm{C}$ & 1.24430 & -1.31856 & -0.42694 \\
\hline $\mathrm{C}$ & -0.17755 & -0.83822 & -0.40157 \\
\hline $\mathrm{H}$ & 1.27559 & -2.39660 & -0.24595 \\
\hline $\mathrm{H}$ & 1.66597 & -1.14868 & -1.42348 \\
\hline $\mathrm{C}$ & -1.17833 & -1.62242 & 0.01755 \\
\hline $\mathrm{H}$ & -0.37370 & 0.16293 & -0.76575 \\
\hline $\mathrm{O}$ & -2.50698 & -1.34855 & 0.06285 \\
\hline $\mathrm{H}$ & -0.99978 & -2.63084 & 0.37873 \\
\hline $\mathrm{C}$ & -2.93630 & -0.05540 & -0.40020 \\
\hline $\mathrm{C}$ & -4.43372 & 0.02413 & -0.28042 \\
\hline $\mathrm{H}$ & -2.62013 & 0.07385 & -1.44112 \\
\hline $\mathrm{H}$ & -2.45351 & 0.72003 & 0.20497 \\
\hline $\mathrm{C}$ & -5.02920 & 0.45961 & 0.90736 \\
\hline $\mathrm{C}$ & -5.25192 & -0.35828 & -1.34757 \\
\hline
\end{tabular}




$\begin{array}{lccc}\text { C } & -6.63966 & -0.30799 & -1.23080 \\ \text { C } & -7.22417 & 0.12674 & -0.04195 \\ \text { C } & -6.41636 & 0.51073 & 1.02796 \\ \text { H } & -4.40273 & 0.75821 & 1.74176 \\ \text { H } & -4.79916 & -0.69814 & -2.27344 \\ \text { H } & -7.26342 & -0.60599 & -2.06626 \\ \text { H } & -8.30383 & 0.16830 & 0.04978 \\ \text { H } & -6.86632 & 0.85117 & 1.95397 \\ \text { O } & 3.76375 & -2.50999 & 0.78882\end{array}$

$\begin{array}{lrrr}\text { X_22 } & & & \\ \mathrm{C} & -3.78218 & -1.98518 & 0.43362 \\ \mathrm{C} & -5.04942 & -2.43623 & 0.06537 \\ \mathrm{C} & -3.39533 & -0.65798 & 0.23051 \\ \mathrm{C} & -5.95986 & -1.56310 & -0.52823 \\ \mathrm{C} & -5.59261 & -0.24104 & -0.74083 \\ \mathrm{C} & -4.32166 & 0.22391 & -0.36480 \\ \mathrm{H} & -3.09314 & -2.68654 & 0.88763 \\ \mathrm{H} & -5.32263 & -3.47045 & 0.24316 \\ \mathrm{H} & -6.94477 & -1.91051 & -0.81867 \\ \mathrm{H} & -6.29215 & 0.45243 & -1.19644 \\ \mathrm{C} & -1.98437 & -0.18120 & 0.56052 \\ \mathrm{C} & -1.85827 & 1.35370 & 0.61723 \\ \mathrm{C} & -2.83026 & 2.18610 & -0.12320 \\ \mathrm{C} & -3.97394 & 1.62033 & -0.56524 \\ \mathrm{C} & -2.52381 & 3.64862 & -0.27053 \\ \mathrm{H} & -4.71166 & 2.23941 & -1.07034 \\ \mathrm{H} & -1.56588 & 3.80260 & -0.77679 \\ \mathrm{H} & -2.43600 & 4.13046 & 0.70765 \\ \mathrm{H} & -3.30493 & 4.15629 & -0.84019 \\ \mathrm{C} & -1.44943 & -0.78332 & 1.87066 \\ \mathrm{H} & -2.13692 & -0.58577 & 2.69737 \\ \mathrm{H} & -0.48494 & -0.34212 & 2.11547 \\ \mathrm{H} & -1.31366 & -1.86355 & 1.78771 \\ \mathrm{C} & -1.07067 & -0.64153 & -0.66297 \\ \mathrm{C} & 0.39189 & -0.32698 & -0.54698 \\ \mathrm{H} & -1.48596 & -0.19066 & -1.57099 \\ \mathrm{H} & -1.20868 & -1.72193 & -0.76016 \\ \mathrm{C} & 1.31332 & -1.29326 & -0.45839 \\ \mathrm{H} & 0.68176 & 0.71622 & -0.53370 \\ \mathrm{O} & 2.66140 & -1.17029 & -0.35976 \\ \mathrm{C} & 1.04150 & -2.34479 & -0.46354 \\ & 3.20465 & 0.16297 & -0.32458 \\ & 4.69909 & 0.06765 & -0.18125\end{array}$




\begin{tabular}{|c|c|c|c|}
\hline $\mathrm{H}$ & 2.76433 & 0.70654 & 0.51784 \\
\hline $\mathrm{H}$ & 2.93260 & 0.68235 & -1.25030 \\
\hline $\mathrm{C}$ & 5.29226 & 0.07312 & 1.08472 \\
\hline $\mathrm{C}$ & 5.51522 & -0.04859 & -1.31073 \\
\hline $\mathrm{C}$ & 6.89787 & -0.15931 & -1.17857 \\
\hline $\mathrm{C}$ & 7.48017 & -0.15346 & 0.08863 \\
\hline $\mathrm{C}$ & 6.67491 & -0.03658 & 1.22082 \\
\hline $\mathrm{H}$ & 4.66732 & 0.16437 & 1.96720 \\
\hline $\mathrm{H}$ & 5.06440 & -0.05262 & -2.29793 \\
\hline $\mathrm{H}$ & 7.52006 & -0.24716 & -2.06237 \\
\hline $\mathrm{H}$ & 8.55636 & -0.23644 & 0.19294 \\
\hline $\mathrm{H}$ & 7.12285 & -0.02876 & 2.20833 \\
\hline $\mathrm{O}$ & -0.92581 & 1.87766 & 1.21246 \\
\hline \multicolumn{4}{|c|}{ X_26 } \\
\hline $\mathrm{C}$ & -3.03493 & -2.09225 & 0.78163 \\
\hline $\mathrm{C}$ & -3.80862 & -2.96814 & 0.02247 \\
\hline $\mathrm{C}$ & -2.87311 & -0.75469 & 0.40995 \\
\hline $\mathrm{C}$ & -4.44467 & -2.51747 & -1.13438 \\
\hline $\mathrm{C}$ & -4.29763 & -1.19182 & -1.51806 \\
\hline $\mathrm{C}$ & -3.51750 & -0.30297 & -0.75861 \\
\hline $\mathrm{H}$ & -2.55092 & -2.46497 & 1.67701 \\
\hline $\mathrm{H}$ & -3.91408 & -4.00102 & 0.33533 \\
\hline $\mathrm{H}$ & -5.04782 & -3.19465 & -1.72832 \\
\hline $\mathrm{H}$ & -4.78659 & -0.82676 & -2.41549 \\
\hline $\mathrm{C}$ & -2.03210 & 0.19469 & 1.25721 \\
\hline $\mathrm{C}$ & -1.93500 & 1.63395 & 0.70329 \\
\hline $\mathrm{C}$ & -2.65210 & 2.01124 & -0.52782 \\
\hline $\mathrm{C}$ & -3.37869 & 1.07935 & -1.18239 \\
\hline $\mathrm{C}$ & -2.52432 & 3.43161 & -0.99662 \\
\hline $\mathrm{H}$ & -3.90232 & 1.36232 & -2.09256 \\
\hline $\mathrm{H}$ & -3.09675 & 3.59439 & -1.91212 \\
\hline $\mathrm{H}$ & -1.47794 & 3.68721 & -1.18843 \\
\hline $\mathrm{H}$ & -2.87805 & 4.13093 & -0.23311 \\
\hline $\mathrm{C}$ & -2.65942 & 0.31544 & 2.67137 \\
\hline $\mathrm{H}$ & -3.69006 & 0.67632 & 2.61609 \\
\hline $\mathrm{H}$ & -2.07914 & 1.01678 & 3.27370 \\
\hline $\mathrm{H}$ & -2.66789 & -0.65470 & 3.17293 \\
\hline $\mathrm{C}$ & -0.56598 & -0.34020 & 1.40959 \\
\hline $\mathrm{C}$ & 0.18792 & -0.52288 & 0.12345 \\
\hline $\mathrm{H}$ & -0.61991 & -1.29257 & 1.94815 \\
\hline $\mathrm{H}$ & -0.03656 & 0.36288 & 2.05653 \\
\hline $\mathrm{C}$ & 1.26515 & 0.19705 & -0.18916 \\
\hline $\mathrm{H}$ & -0.16225 & -1.27224 & -0.58103 \\
\hline
\end{tabular}




$\begin{array}{rrrr}\mathrm{O} & 1.92401 & 0.04567 & -1.37649 \\ \mathrm{H} & 1.66612 & 0.95881 & 0.47626 \\ \mathrm{C} & 3.16262 & 0.75679 & -1.49457 \\ \mathrm{C} & 4.29687 & 0.14258 & -0.70135 \\ \mathrm{H} & 3.02021 & 1.80472 & -1.20602 \\ \mathrm{H} & 3.38892 & 0.73452 & -2.56349 \\ \mathrm{C} & 4.48504 & -1.24392 & -0.68181 \\ \mathrm{C} & 5.19400 & 0.95706 & -0.00587 \\ \mathrm{C} & 6.26782 & 0.40076 & 0.68976 \\ \mathrm{C} & 6.44815 & -0.98044 & 0.70426 \\ \mathrm{C} & 5.55139 & -1.80143 & 0.01884 \\ \mathrm{H} & 3.78638 & -1.88409 & -1.20876 \\ \mathrm{H} & 5.05166 & 2.03333 & -0.00449 \\ \mathrm{H} & 6.95531 & 1.04560 & 1.22608 \\ \mathrm{H} & 7.27817 & -1.41622 & 1.24921 \\ \mathrm{H} & 5.68464 & -2.87770 & 0.02987 \\ \mathrm{O} & -1.26534 & 2.46075 & 1.30970\end{array}$




\section{References:}

[1] Schrödinger Release 2017-4: MacroModel, Schrödinger, LLC, New York, NY, 2017.

[2] Gaussian 16, Revision A.03, Frisch, M. J.; Trucks, G. W.; Schlegel, H. B.; Scuseria, G. E.; Robb, M. A.; Cheeseman, J. R.; Scalmani, G.; Barone, V.; Petersson, G. A.; Nakatsuji, H.; Li, X.; Caricato, M.; Marenich, A. V.; Bloino, J.; Janesko, B. G.; Gomperts, R.; Mennucci, B.; Hratchian, H. P.; Ortiz, J. V.; Izmaylov, A. F.; Sonnenberg, J. L.; Williams-Young, D.; Ding, F.; Lipparini, F.; Egidi, F.; Goings, J.; Peng, B.; Petrone, A.; Henderson, T.; Ranasinghe, D.; Zakrzewski, V. G.; Gao, J.; Rega, N.; Zheng, G.; Liang, W.; Hada, M.; Ehara, M.; Toyota, K.; Fukuda, R.; Hasegawa, J.; Ishida, M.; Nakajima, T.; Honda, Y.; Kitao, O.; Nakai, H.; Vreven, T.; Throssell, K.; Montgomery, J. A.; Peralta, J. E. Jr.; Ogliaro, F.; Bearpark, M. J.; Heyd, J. J.; Brothers, E. N.; Kudin, K. N.; Staroverov, V. N.; Keith, T. A.; Kobayashi, R.; Normand, J.; Raghavachari, K.; Rendell, A. P.; Burant, J. C.; Iyengar, S. S.; Tomasi, J.; Cossi, M.; Millam, J. M.; Klene, M.; Adamo, C.; Cammi, R.; Ochterski, J. W.; Martin, R. L.; Morokuma, K.; Farkas, O.; Foresman, J. B.; Fox, D. J. Gaussian, Inc., Wallingford CT, 2016.

[3] Becke, A. D. J. Chem. Phys. 1993, 98, 5648.

[4] Lee, C.; Yang, W.; Parr, R. G. Phys. Rev. B 1988, 37, 785.

[5] Marenich, A. V.; Cramer, C. J.; Truhlar, D. G. J. Phys. Chem. B. 2009, 113, 6378.

[6] Zhao, Y.; Truhlar, D. G. Phys. Chem. Chem. Phys. 2008, 10, 2813. 
IV NMR and HPLC spectra:

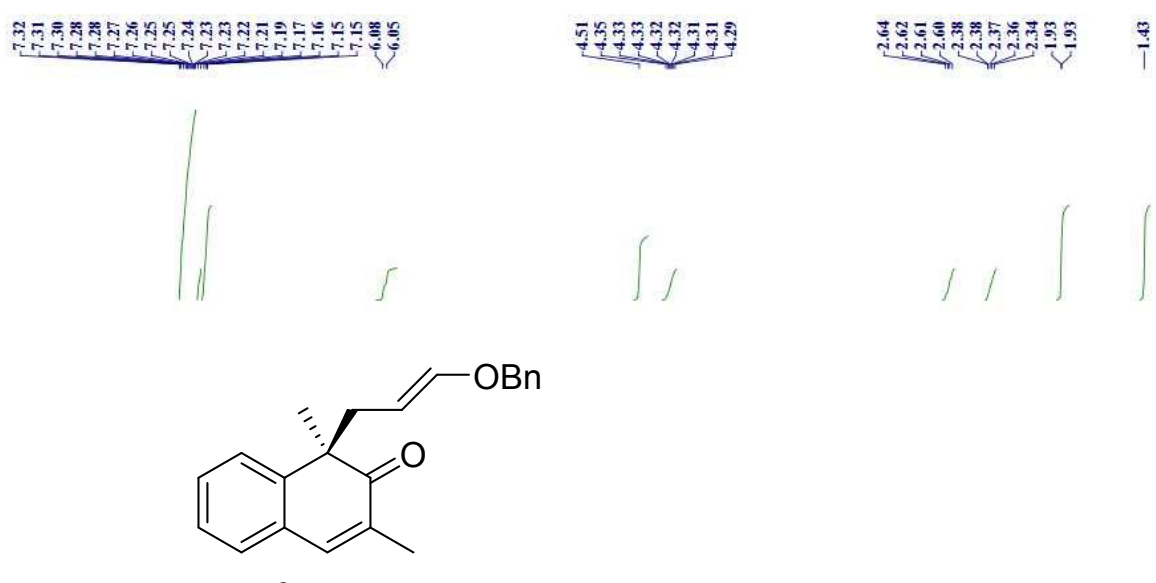

3a

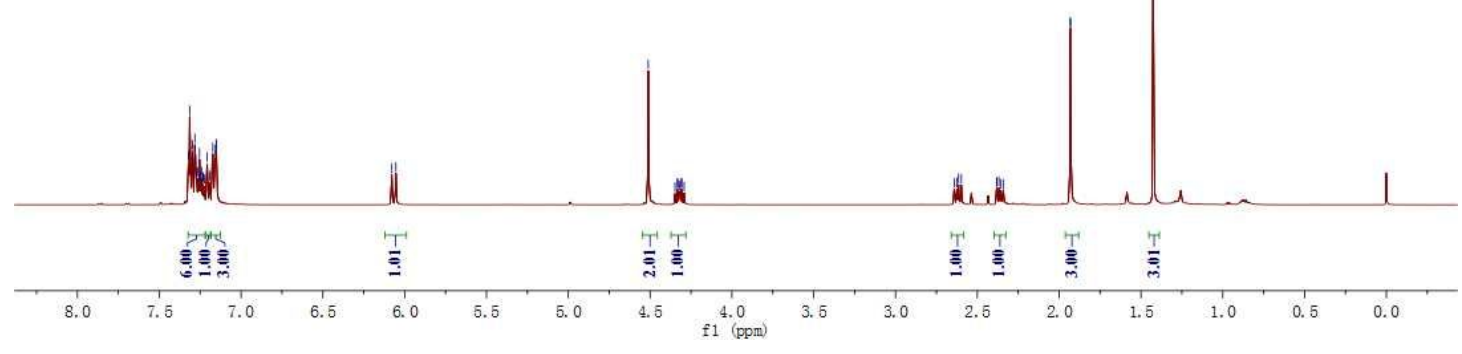

${ }^{1} \mathrm{H}$ NMR Spectra of $3 a\left(500 \mathrm{MHz}, \mathrm{CDCl}_{3}\right)$

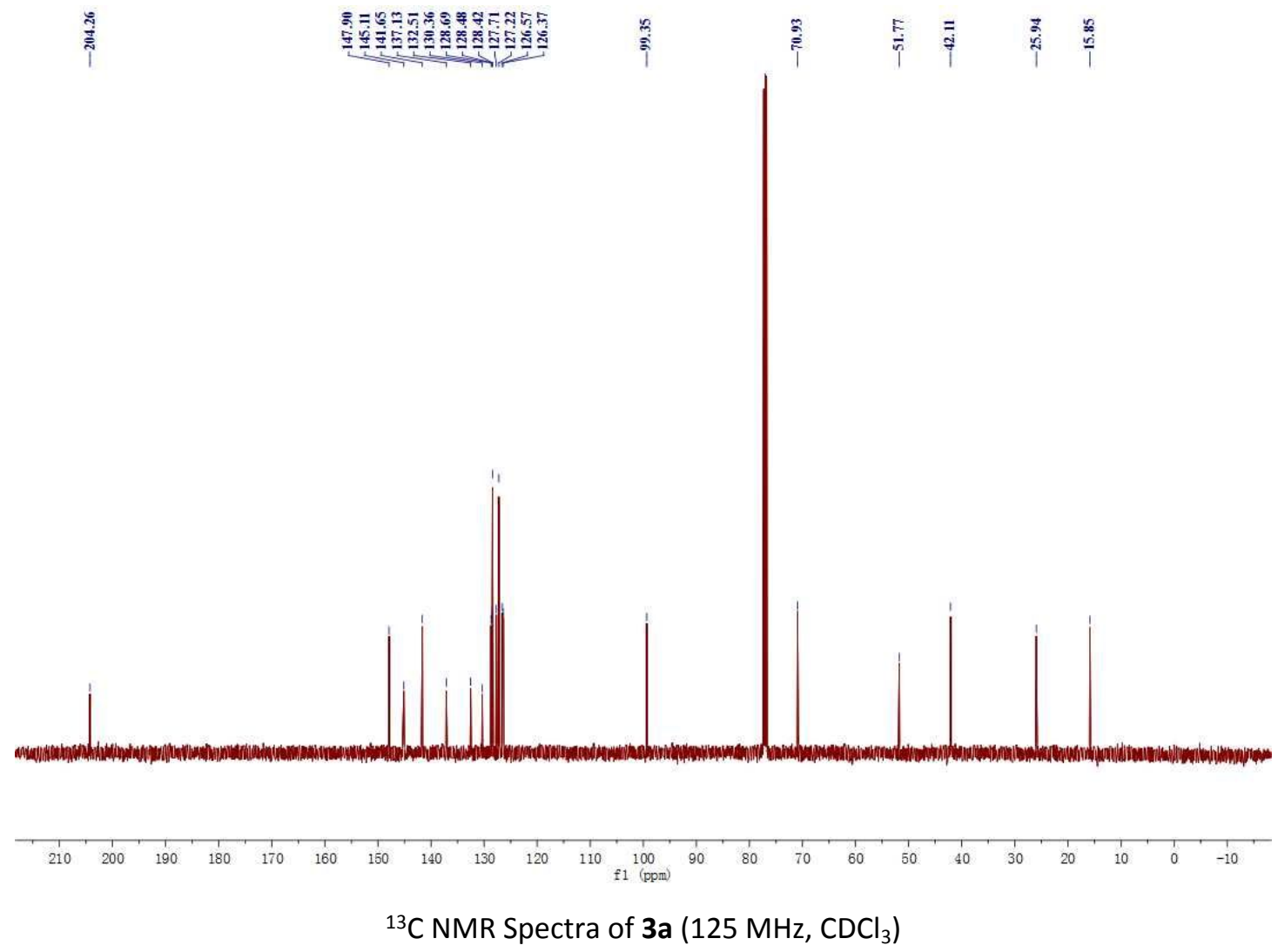




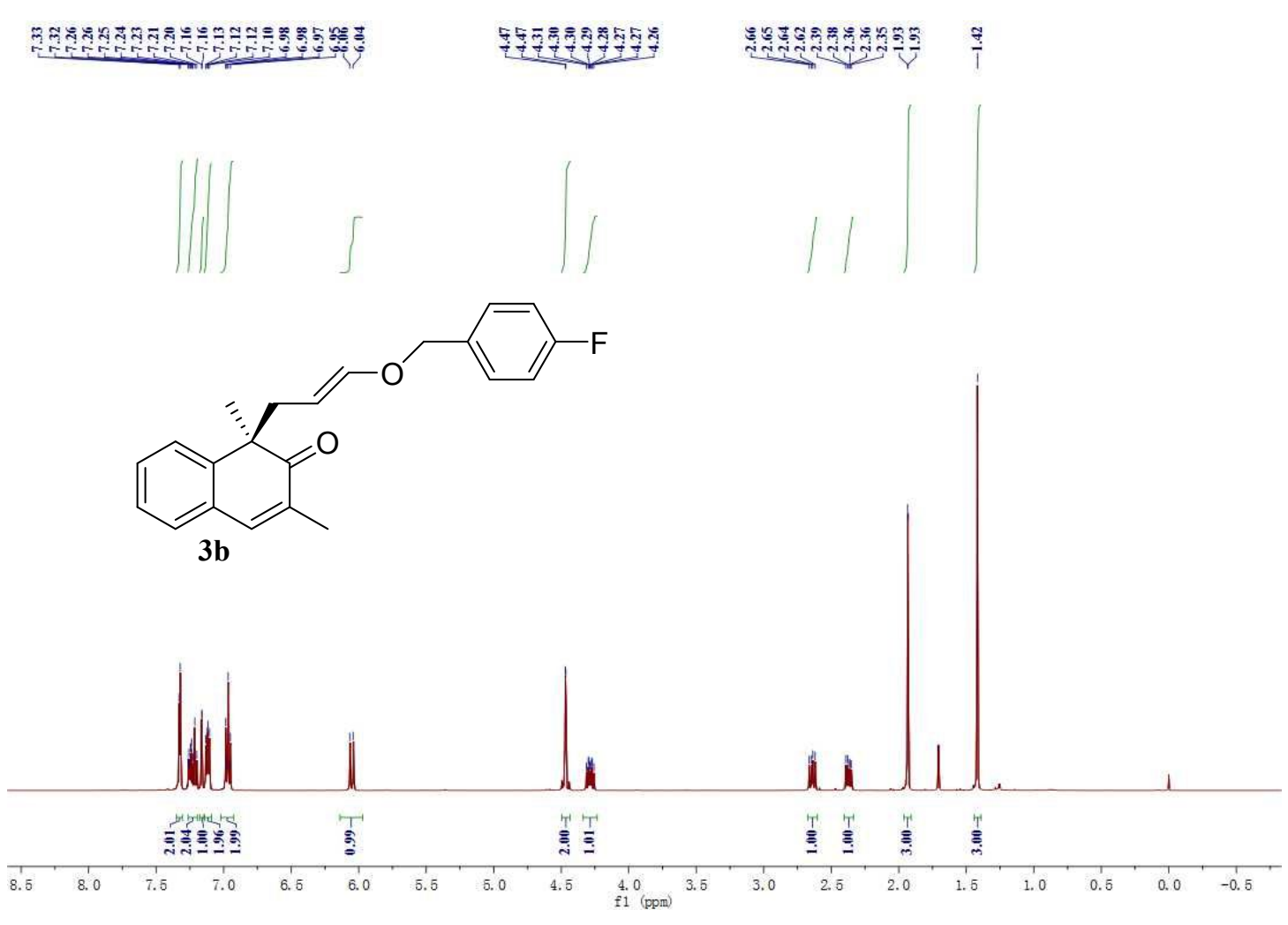

${ }^{1} \mathrm{H}$ NMR Spectra of $\mathbf{3 b}\left(500 \mathrm{MHz}, \mathrm{CDCl}_{3}\right)$

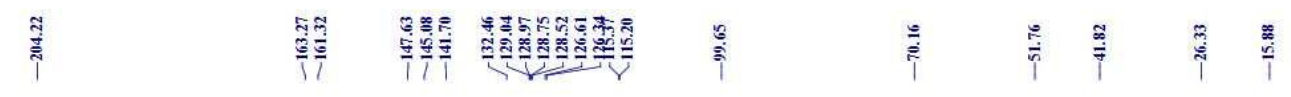
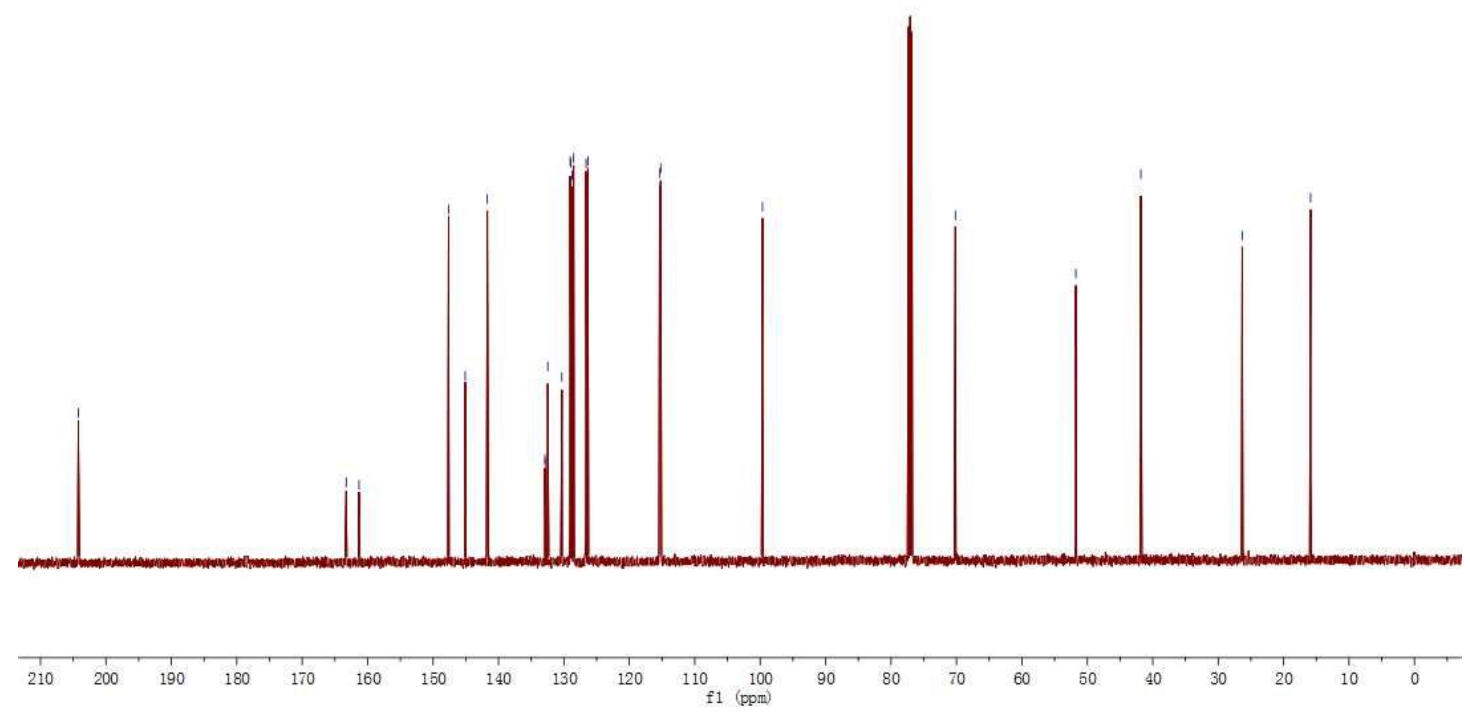

${ }^{13} \mathrm{C}$ NMR Spectra of $\mathbf{3 b}\left(125 \mathrm{MHz}, \mathrm{CDCl}_{3}\right)$ 


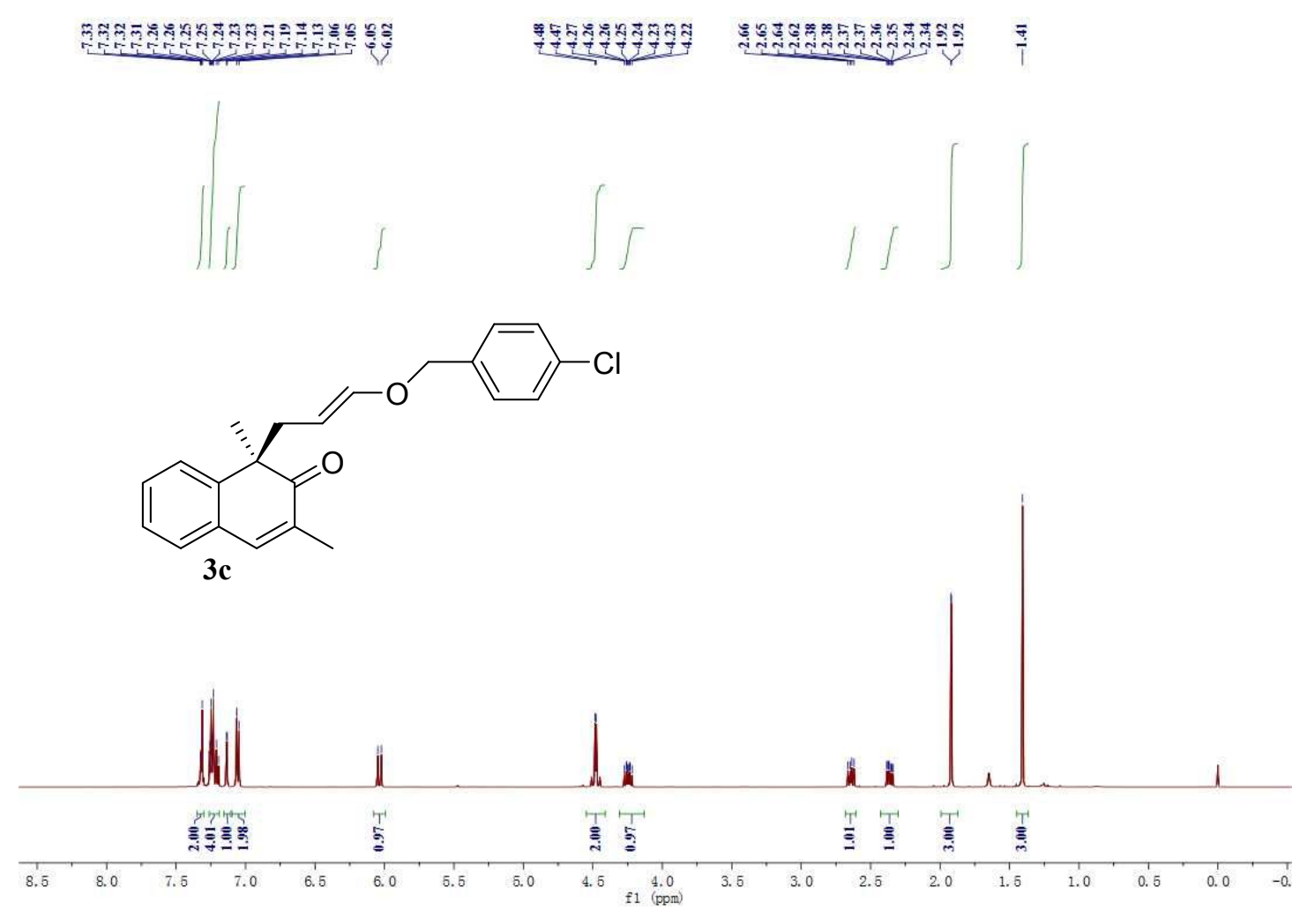

${ }^{1} \mathrm{H}$ NMR Spectra of $\mathbf{3 c}\left(500 \mathrm{MHz}, \mathrm{CDCl}_{3}\right)$

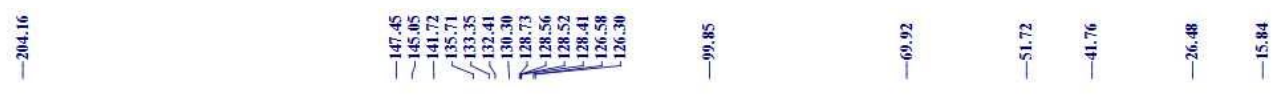

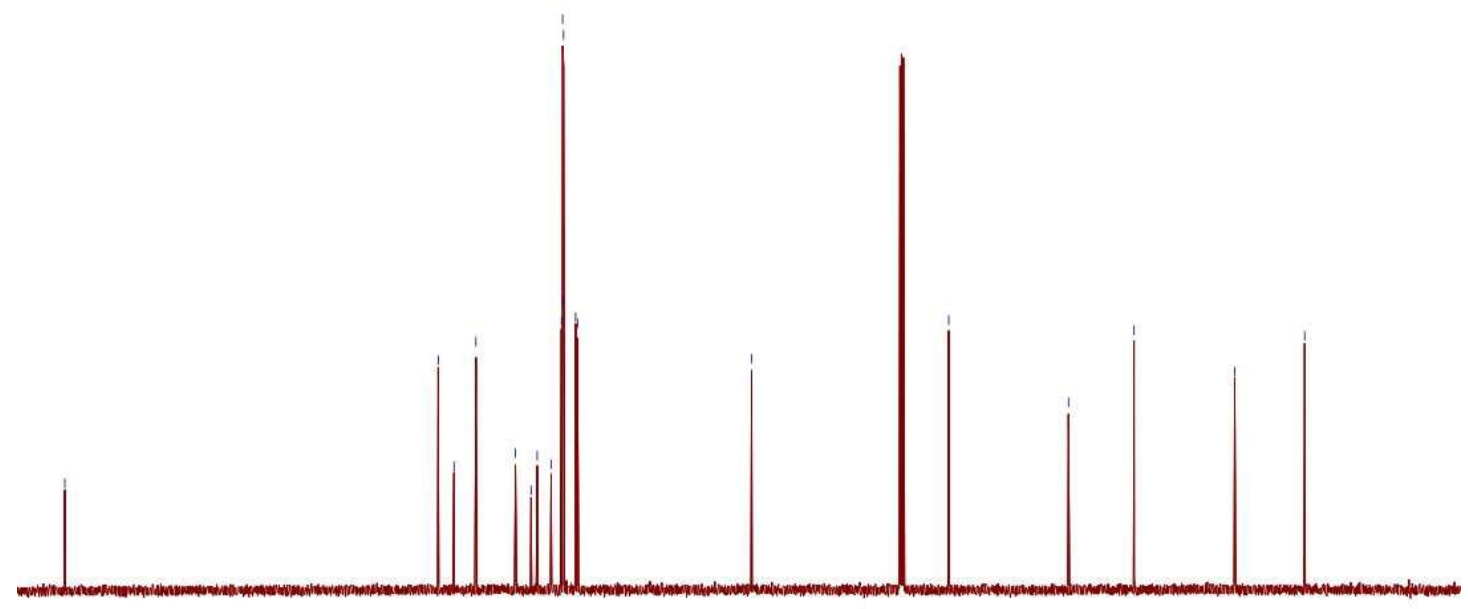

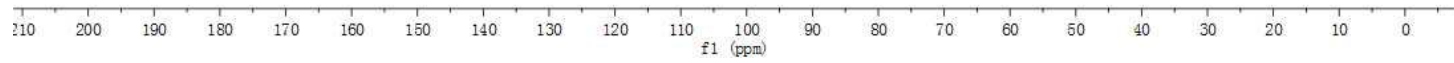

${ }^{13} \mathrm{C}$ NMR Spectra of $3 \mathrm{c}\left(125 \mathrm{MHz}, \mathrm{CDCl}_{3}\right)$ 


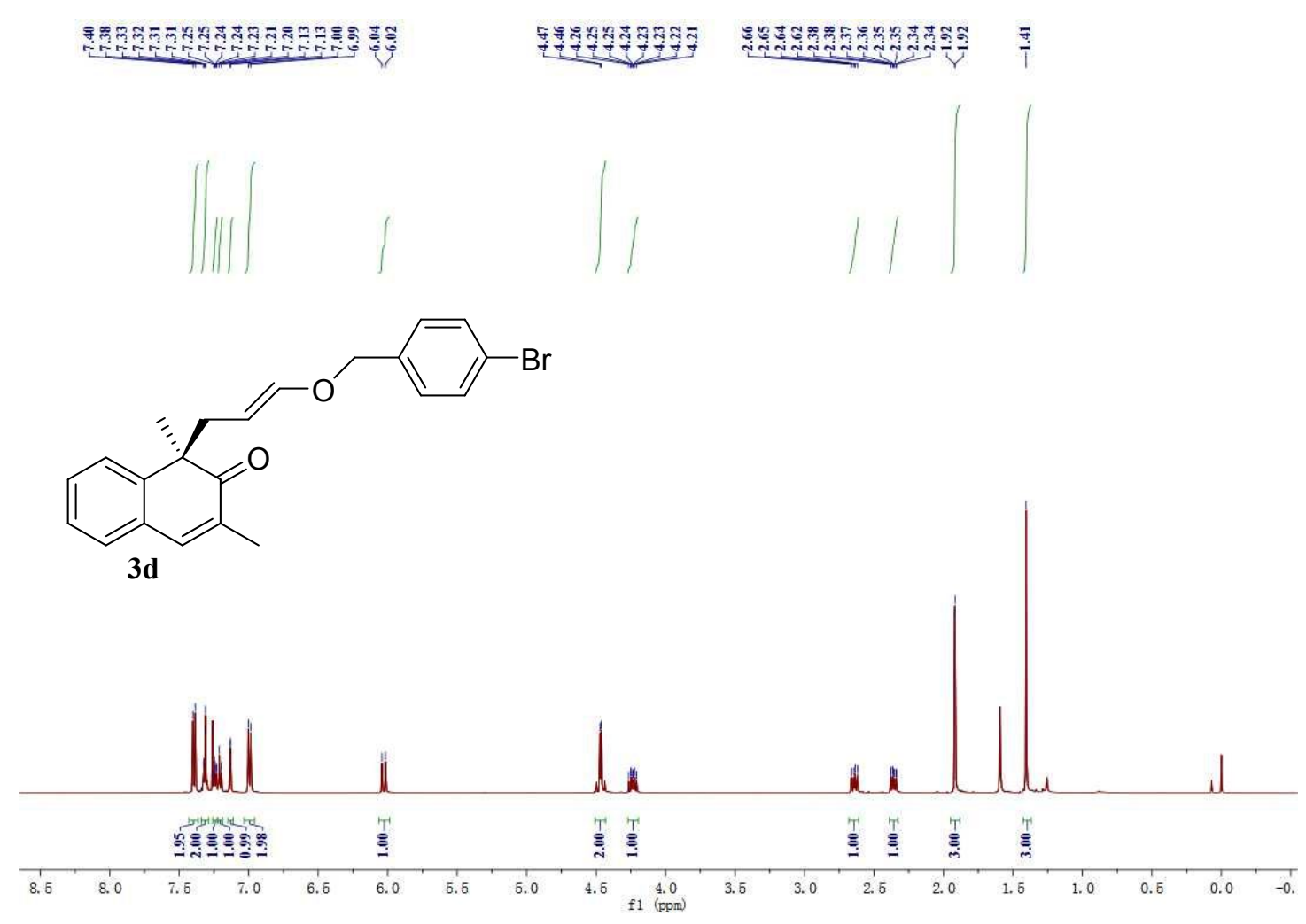

${ }^{1} \mathrm{H}$ NMR Spectra of $\mathbf{3 d}\left(500 \mathrm{MHz}, \mathrm{CDCl}_{3}\right)$

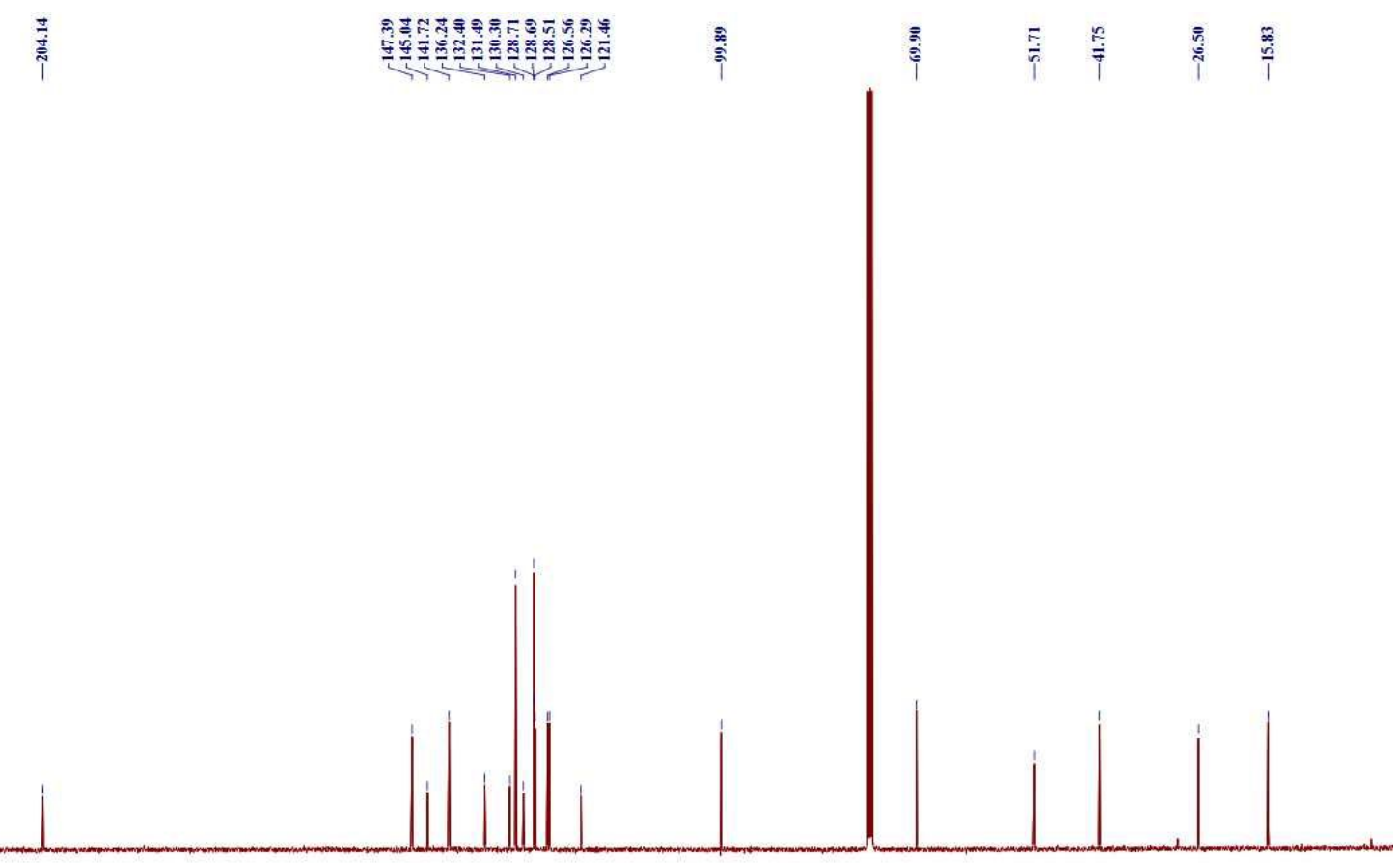

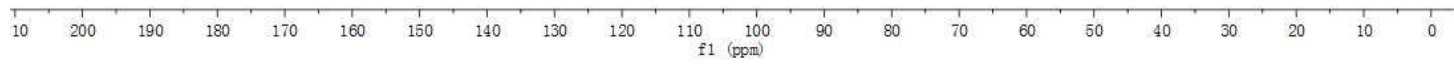

${ }^{13} \mathrm{C}$ NMR Spectra of $\mathbf{3 d}\left(125 \mathrm{MHz}, \mathrm{CDCl}_{3}\right)$ 


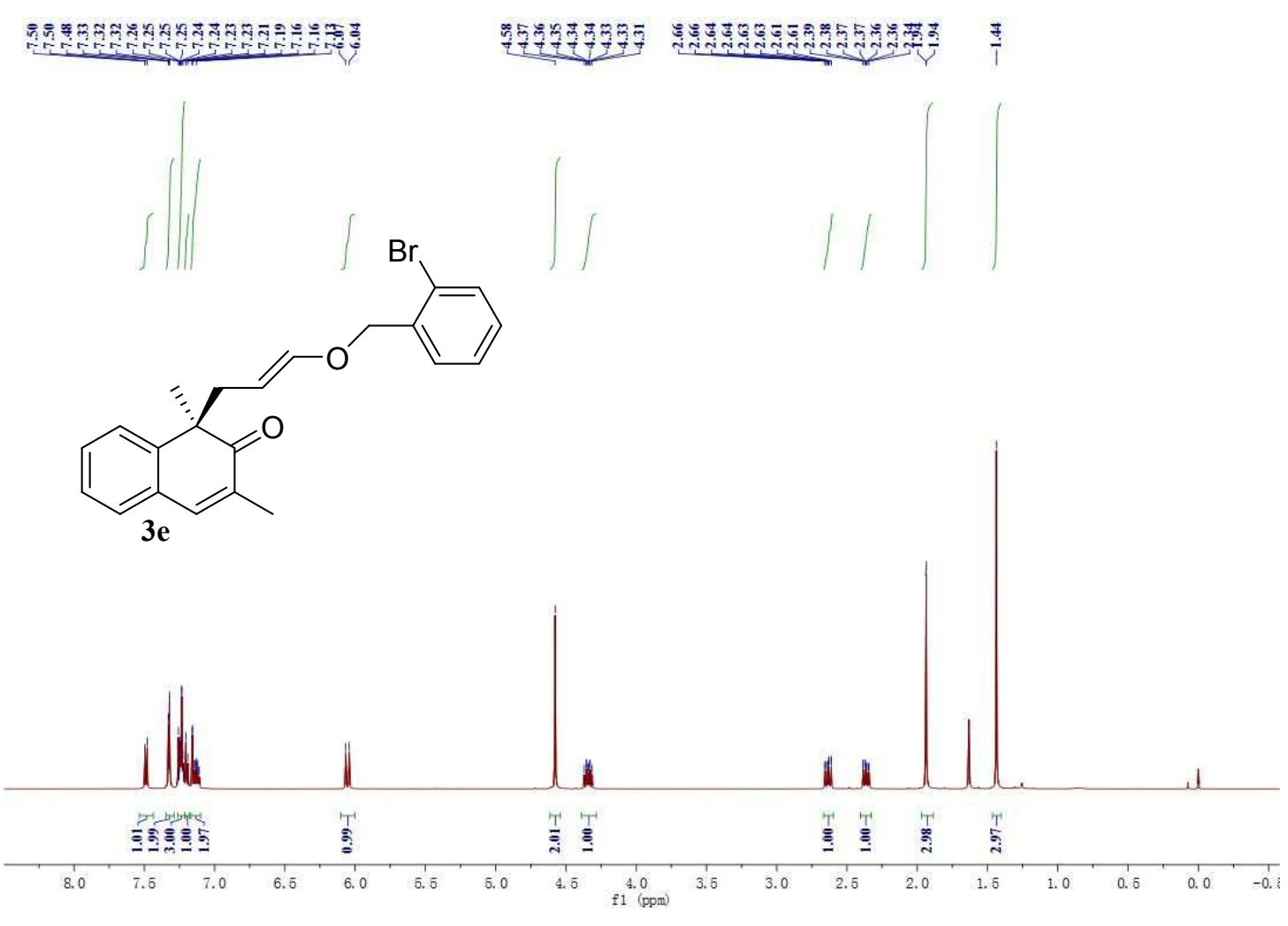

${ }^{1} \mathrm{H}$ NMR Spectra of $3 e\left(500 \mathrm{MHz}, \mathrm{CDCl}_{3}\right)$
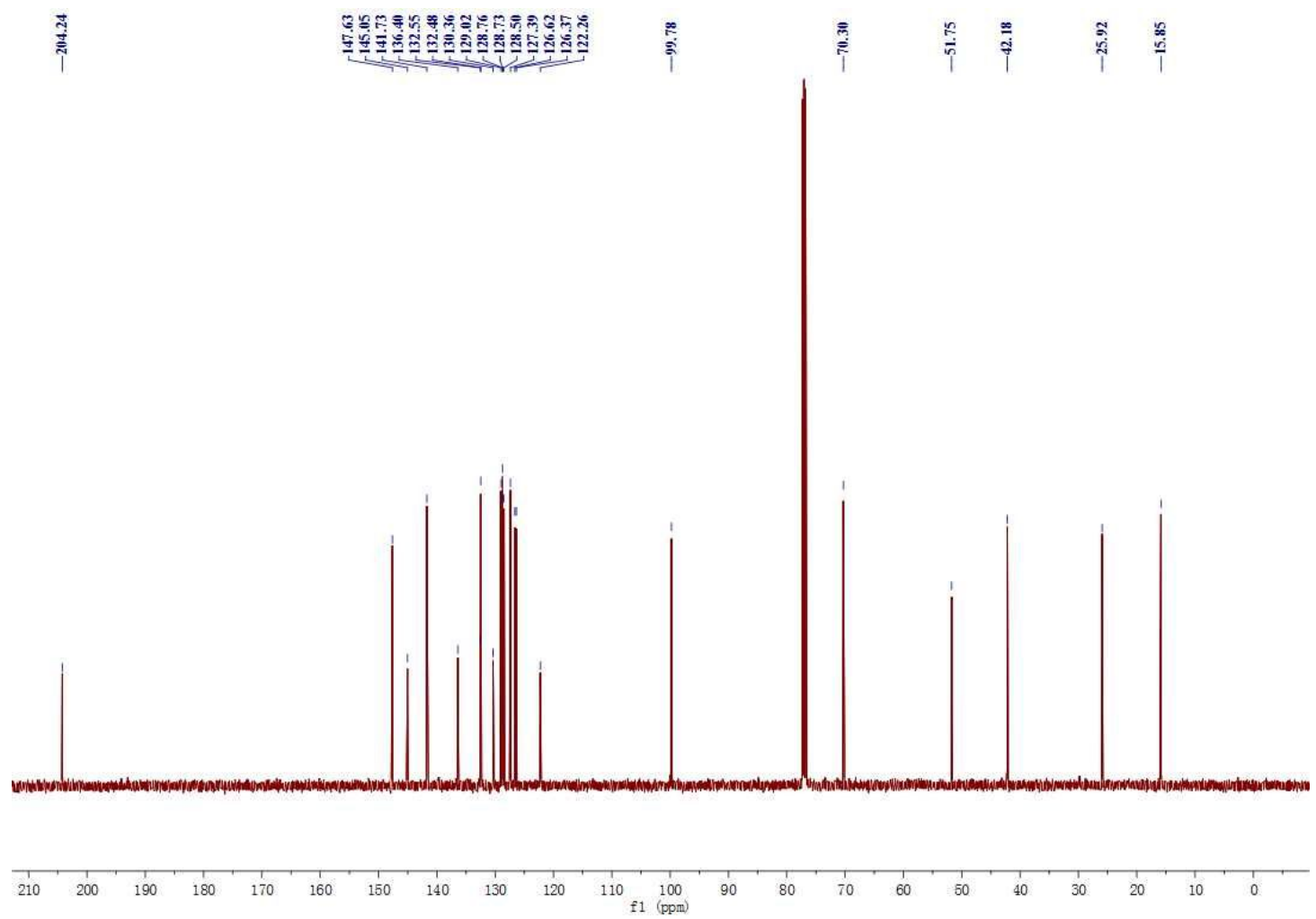

${ }^{13} \mathrm{C}$ NMR Spectra of $3 e\left(125 \mathrm{MHz}, \mathrm{CDCl}_{3}\right)$ 


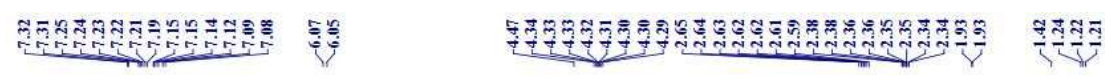
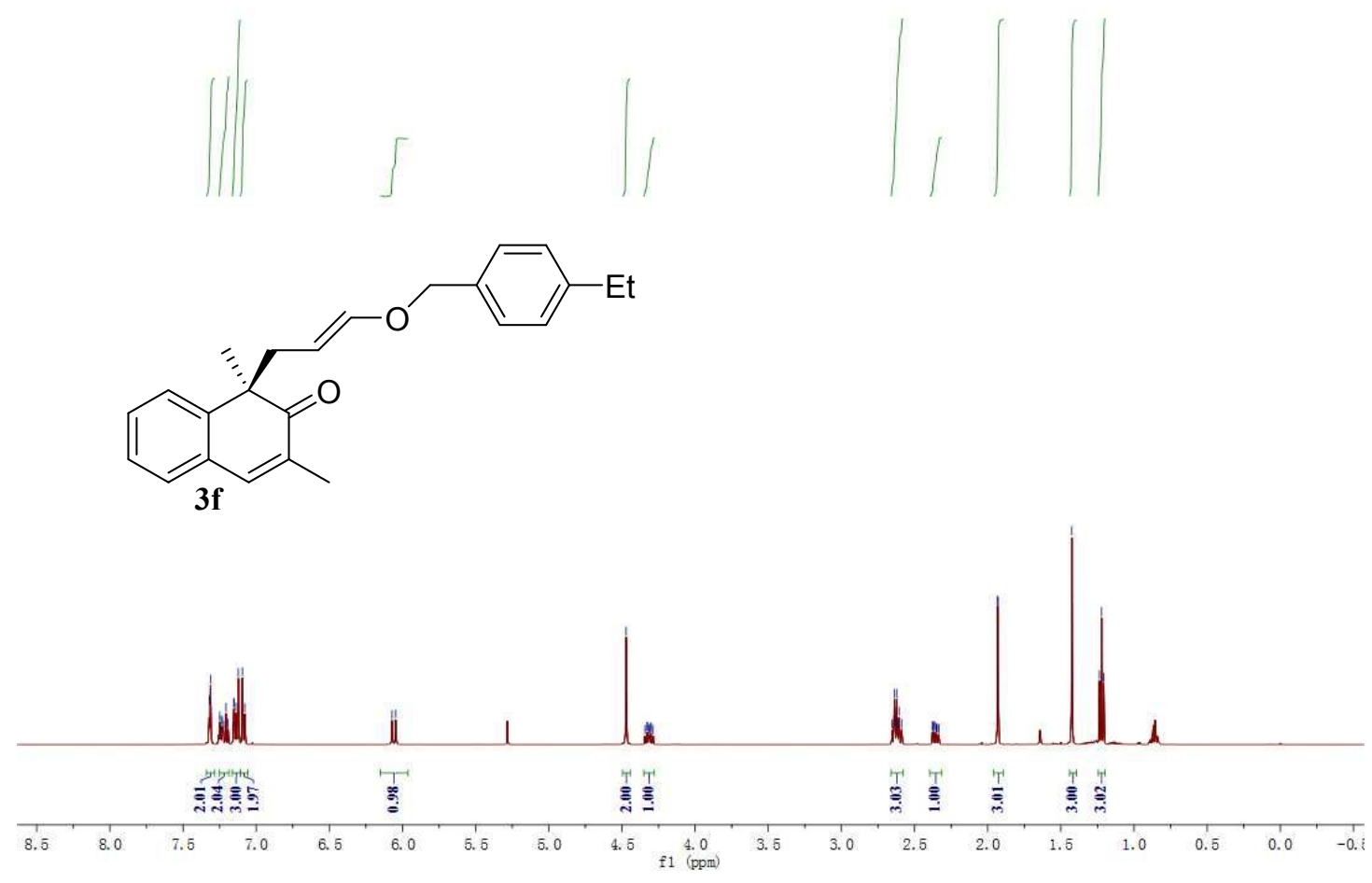

${ }^{1} \mathrm{H}$ NMR Spectra of $3 f\left(500 \mathrm{MHz}, \mathrm{CDCl}_{3}\right)$

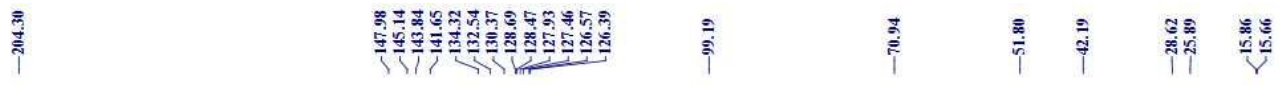
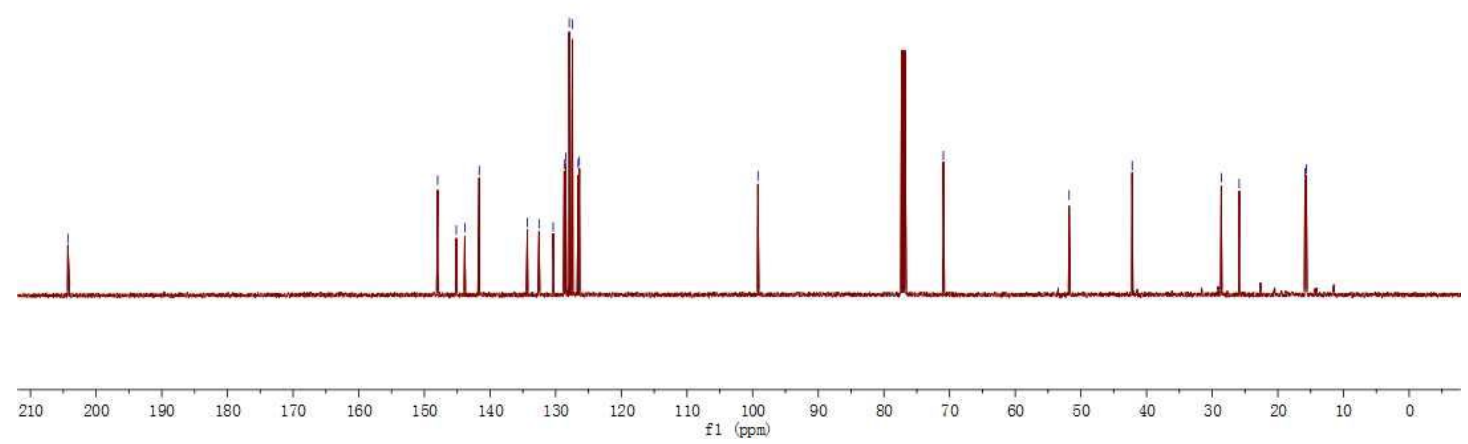

${ }^{13} \mathrm{C}$ NMR Spectra of $\mathbf{3 f}\left(125 \mathrm{MHz}, \mathrm{CDCl}_{3}\right)$ 


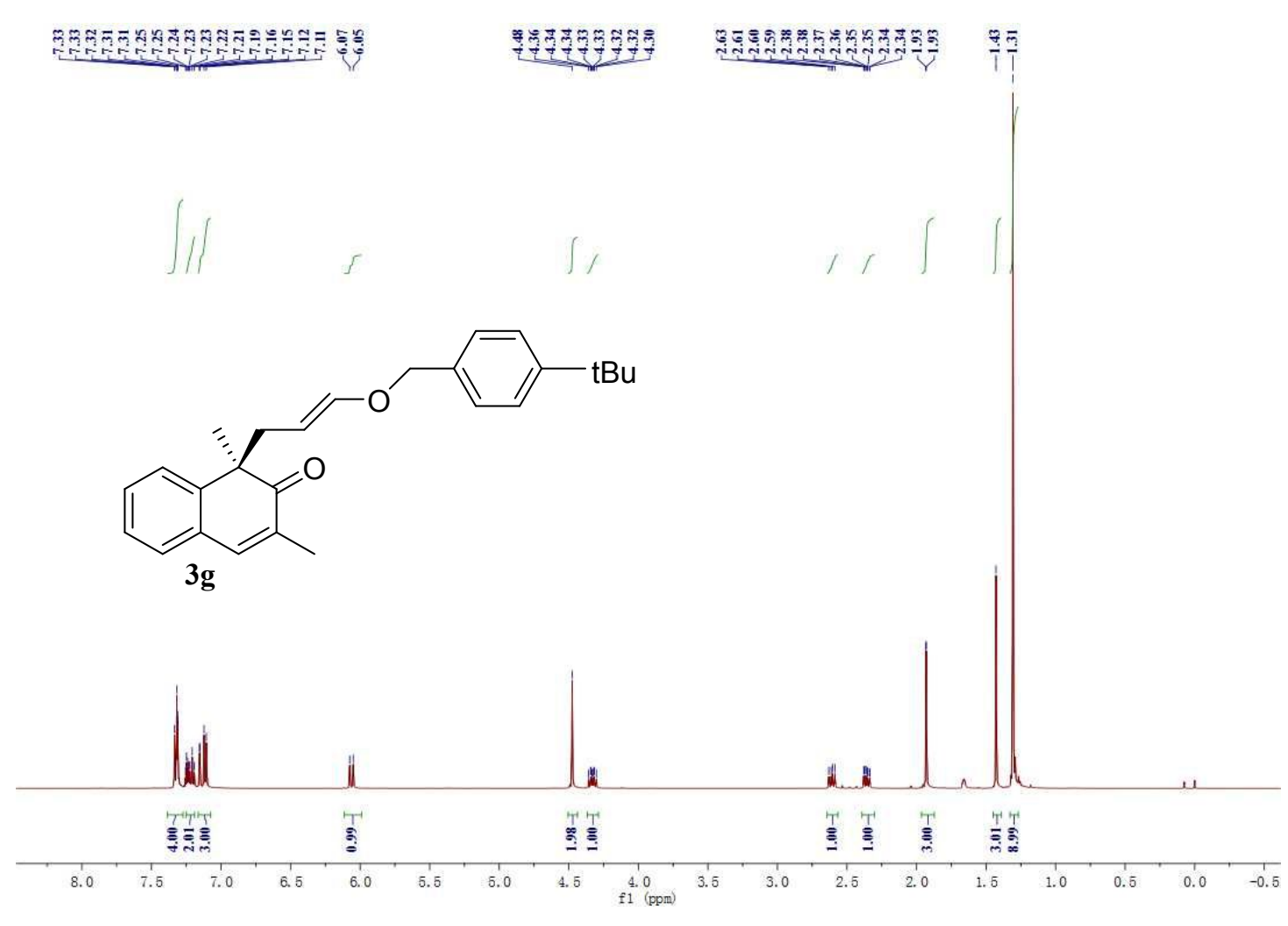

${ }^{1} \mathrm{H}$ NMR Spectra of $\mathbf{3 g}\left(500 \mathrm{MHz} \mathrm{CDCl}_{3}\right)$
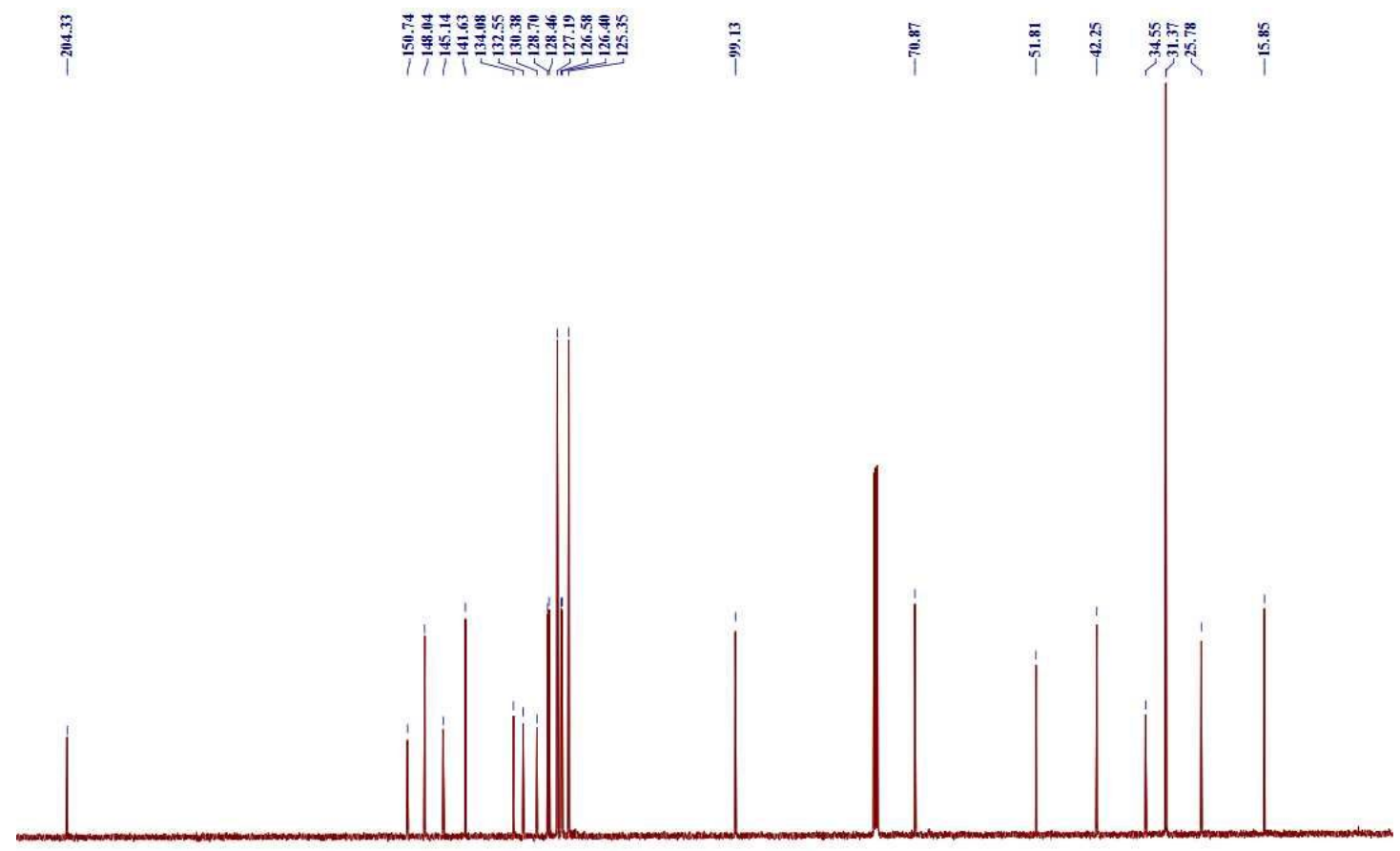

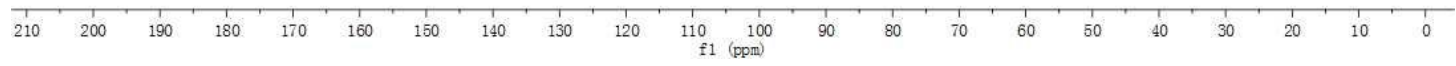

${ }^{13} \mathrm{C}$ NMR Spectra of $\mathbf{3 g}\left(125 \mathrm{MHz}, \mathrm{CDCl}_{3}\right)$ 


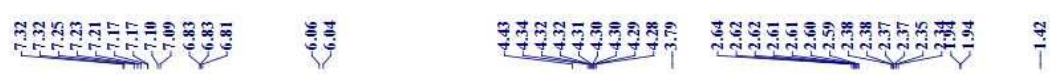

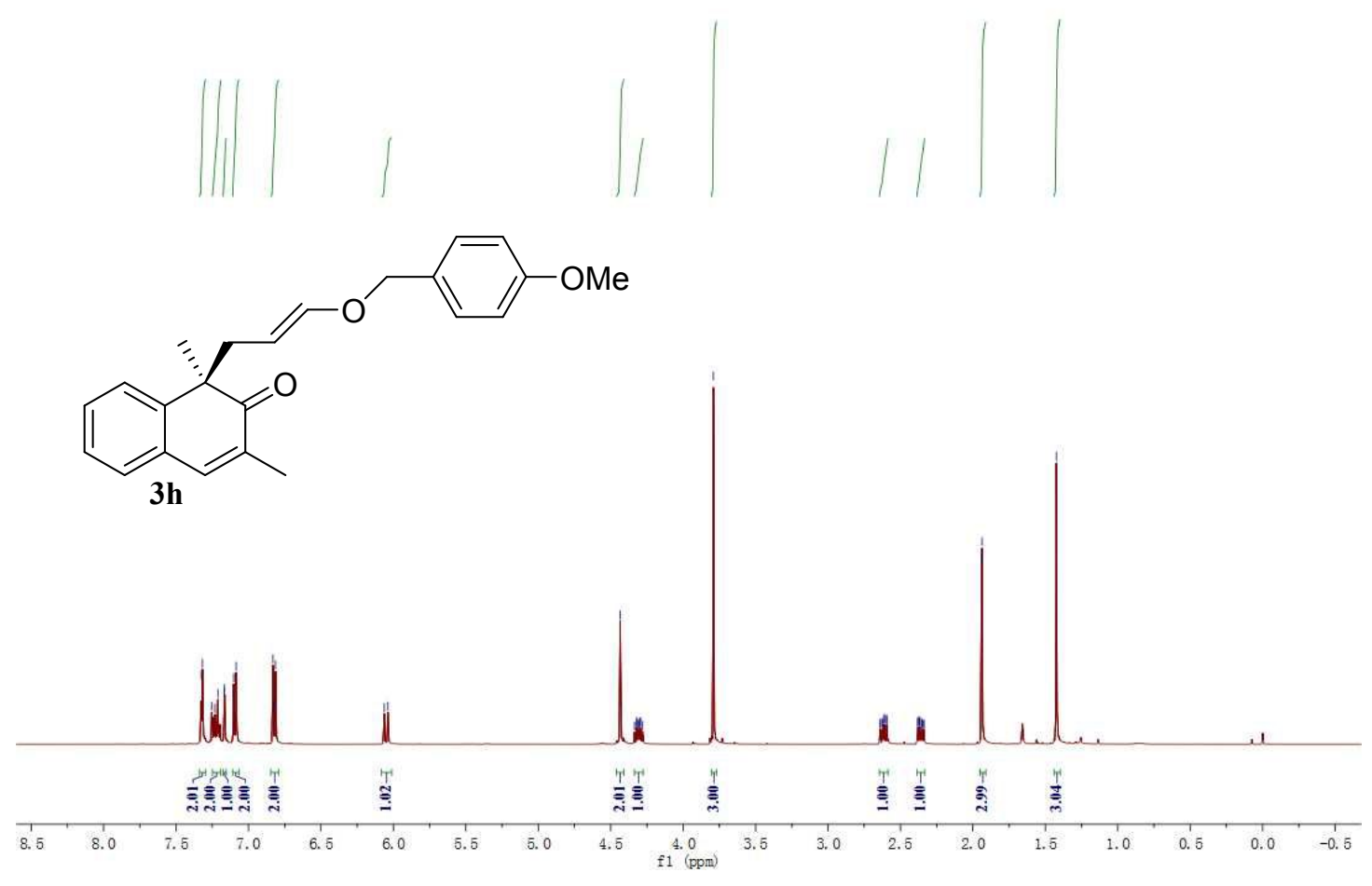

${ }^{1} \mathrm{H}$ NMR Spectra of $\mathbf{3 h}\left(500 \mathrm{MHz}, \mathrm{CDCl}_{3}\right)$
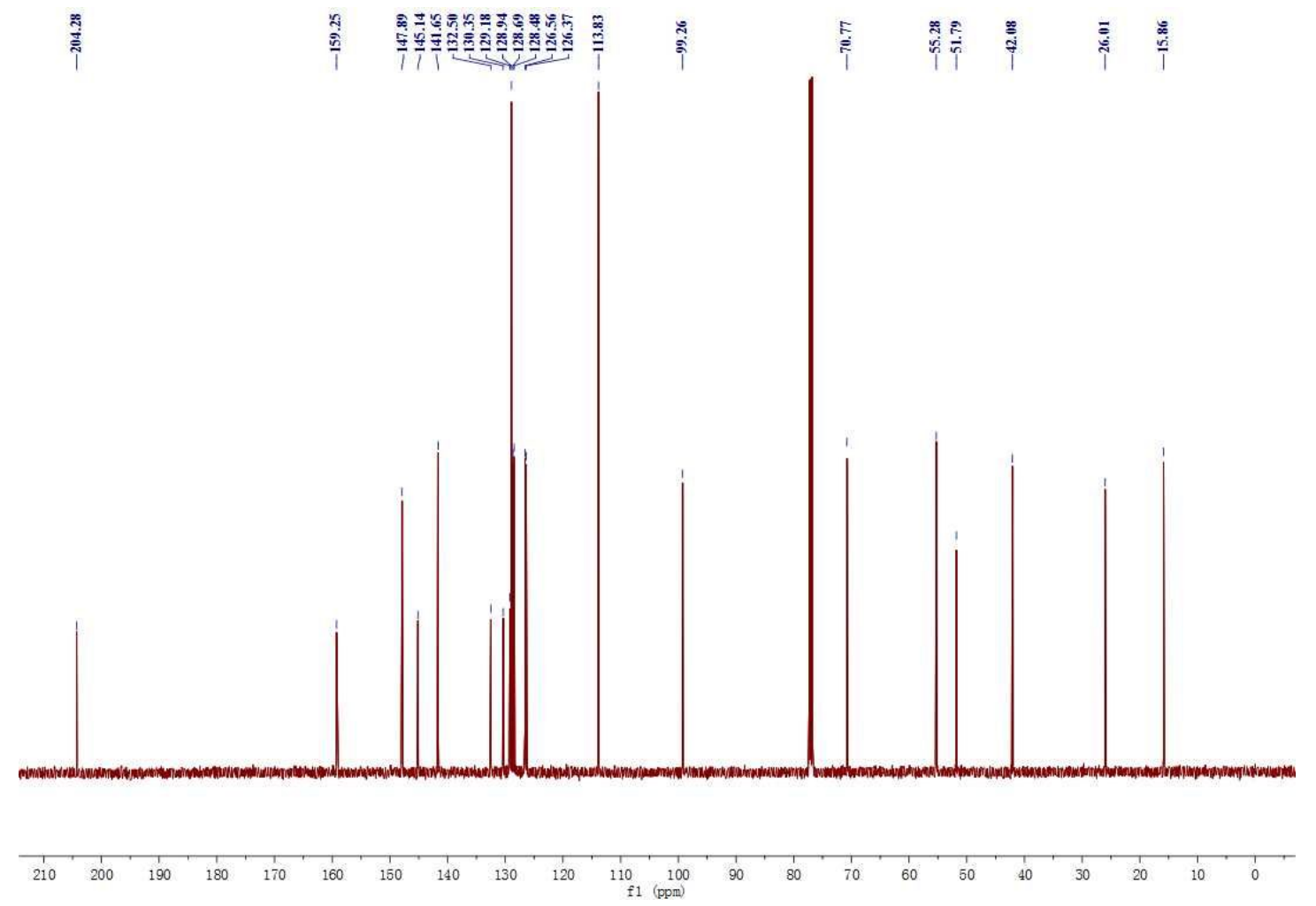

${ }^{13} \mathrm{C}$ NMR Spectra of $3 \mathbf{h}\left(125 \mathrm{MHz}, \mathrm{CDCl}_{3}\right)$ 


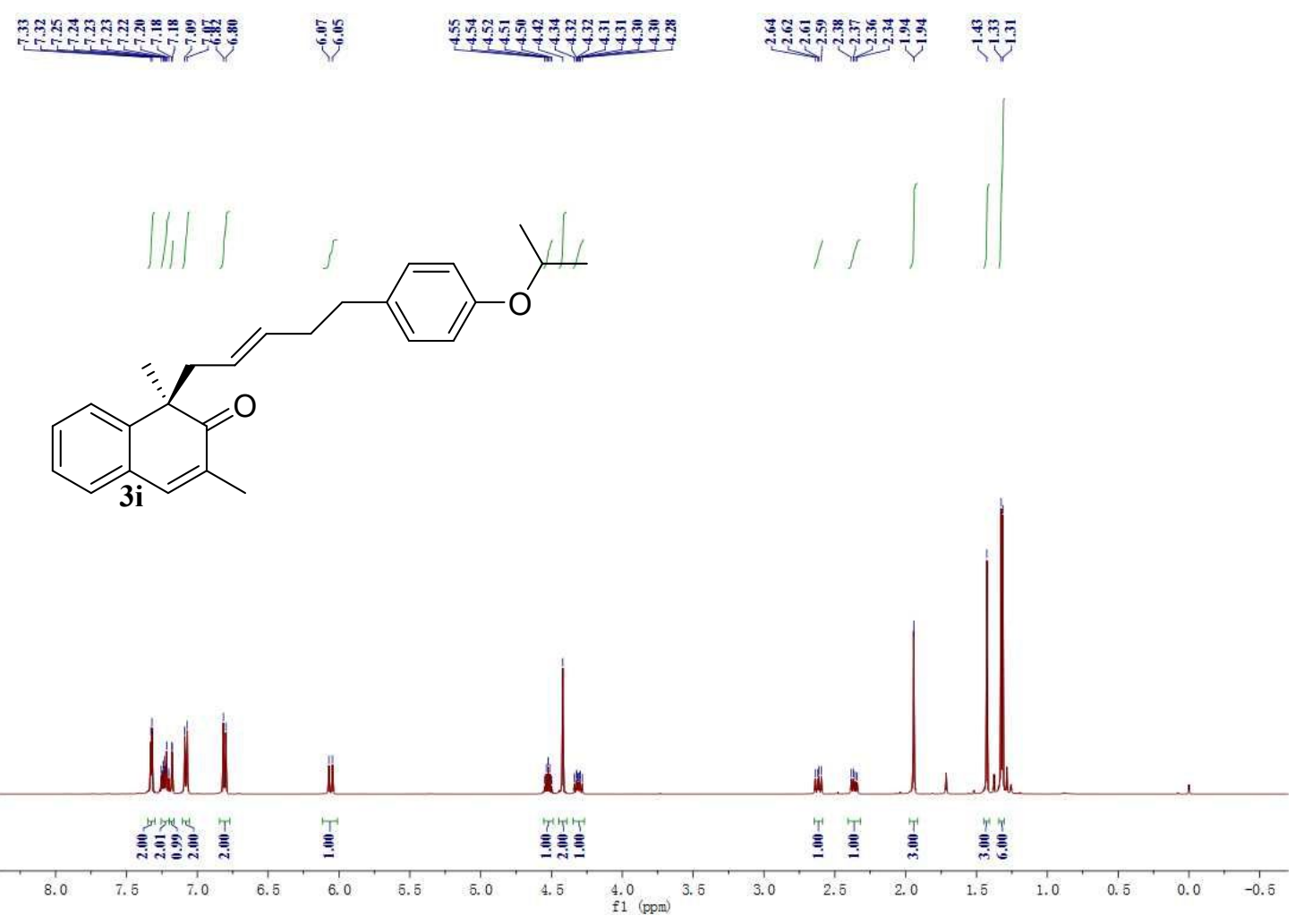

${ }^{1} \mathrm{H}$ NMR Spectra of $\mathbf{3 i}\left(500 \mathrm{MHz}, \mathrm{CDCl}_{3}\right)$

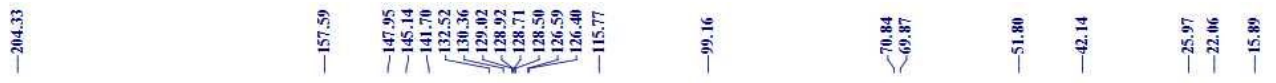
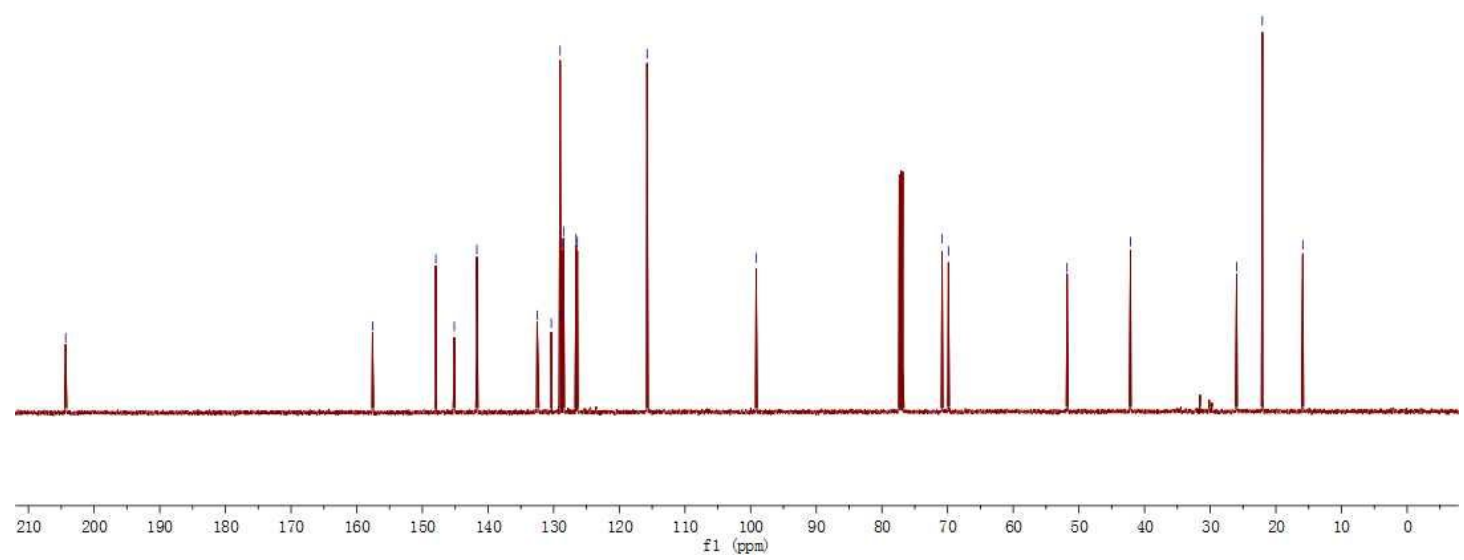

${ }^{13} \mathrm{C}$ NMR Spectra of $\mathbf{3 i}\left(125 \mathrm{MHz}, \mathrm{CDCl}_{3}\right)$ 


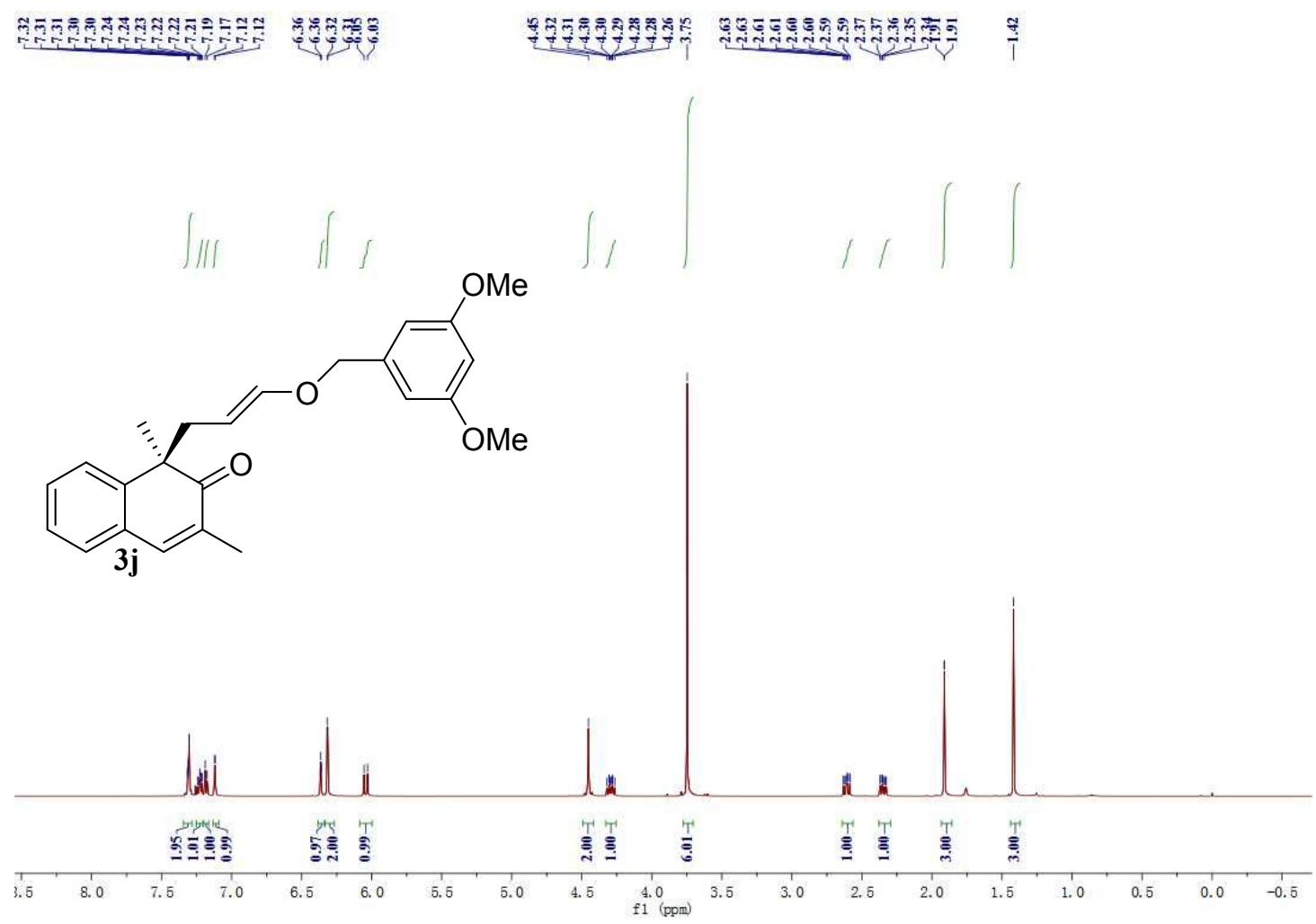

${ }^{1} \mathrm{H}$ NMR Spectra of $\mathbf{3 j}\left(500 \mathrm{MHz}, \mathrm{CDCl}_{3}\right)$

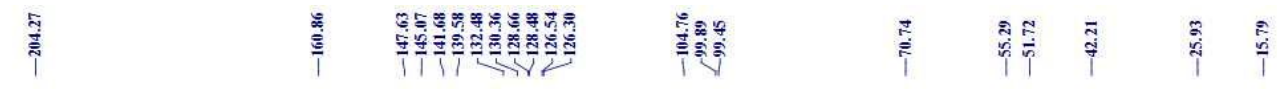
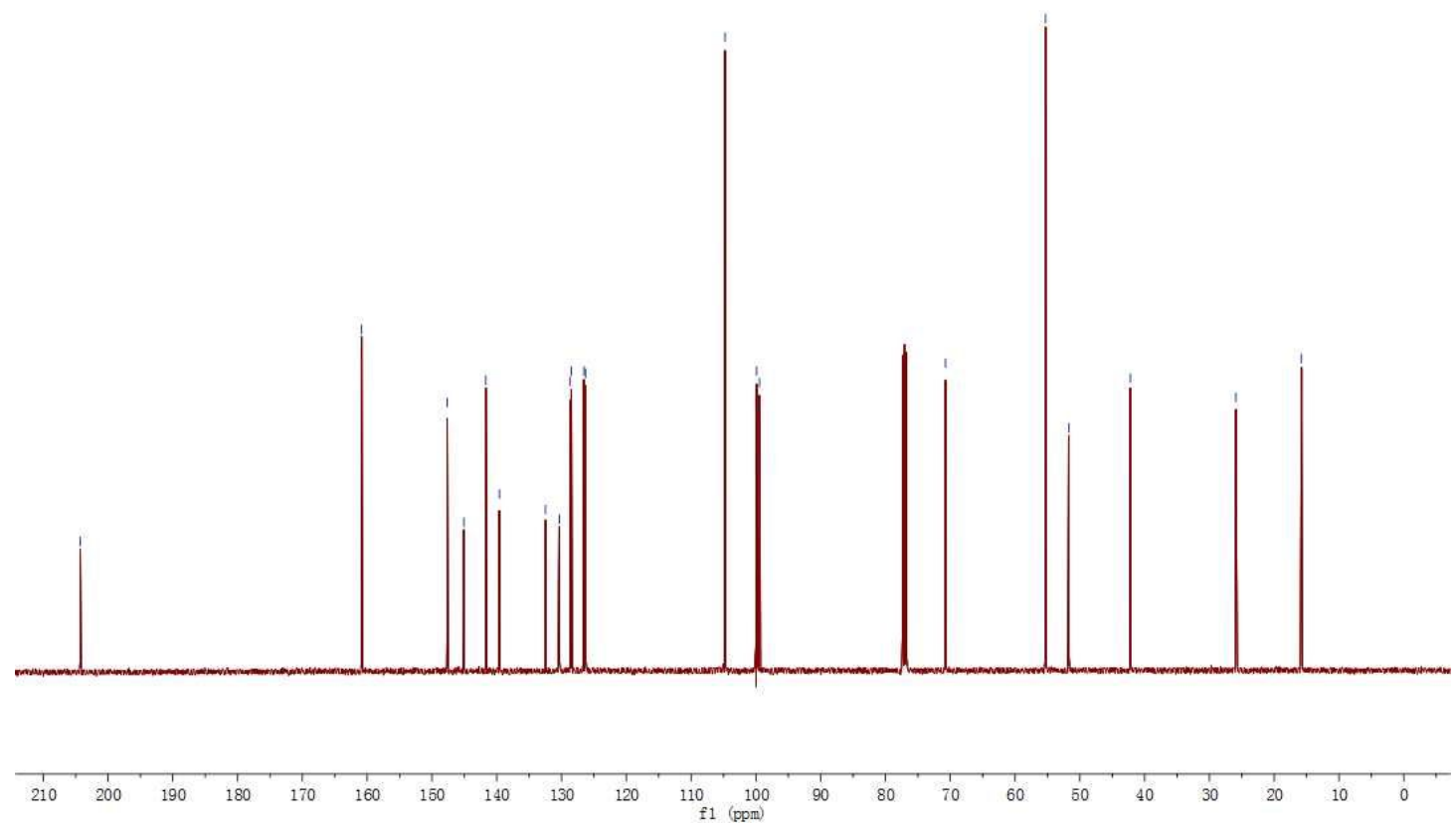

${ }^{13} \mathrm{C}$ NMR Spectra of $\mathbf{3 j}\left(125 \mathrm{MHz}, \mathrm{CDCl}_{3}\right)$ 


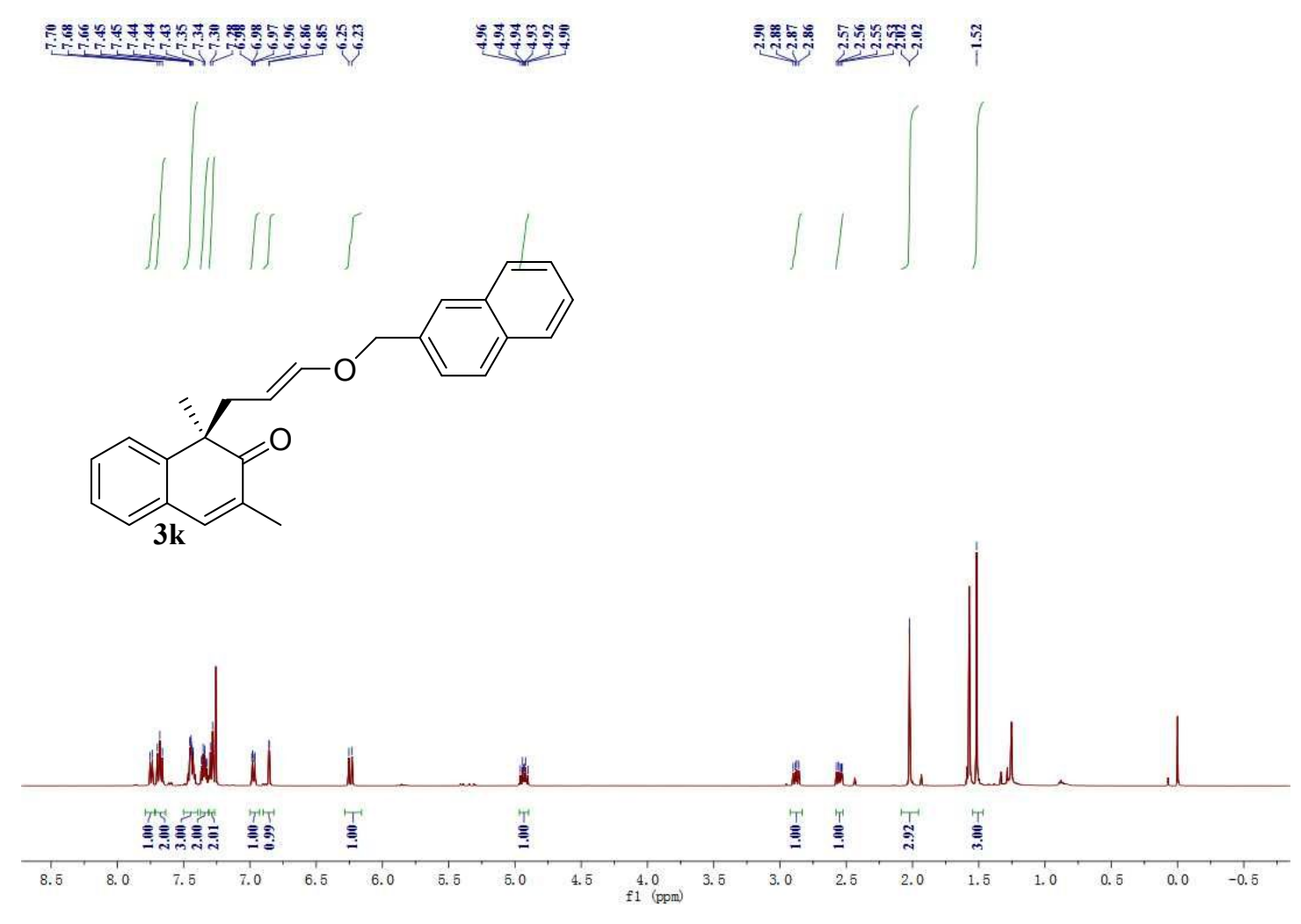

${ }^{1} \mathrm{H}$ NMR Spectra of $\mathbf{3 k}\left(500 \mathrm{MHz}, \mathrm{CDCl}_{3}\right)$

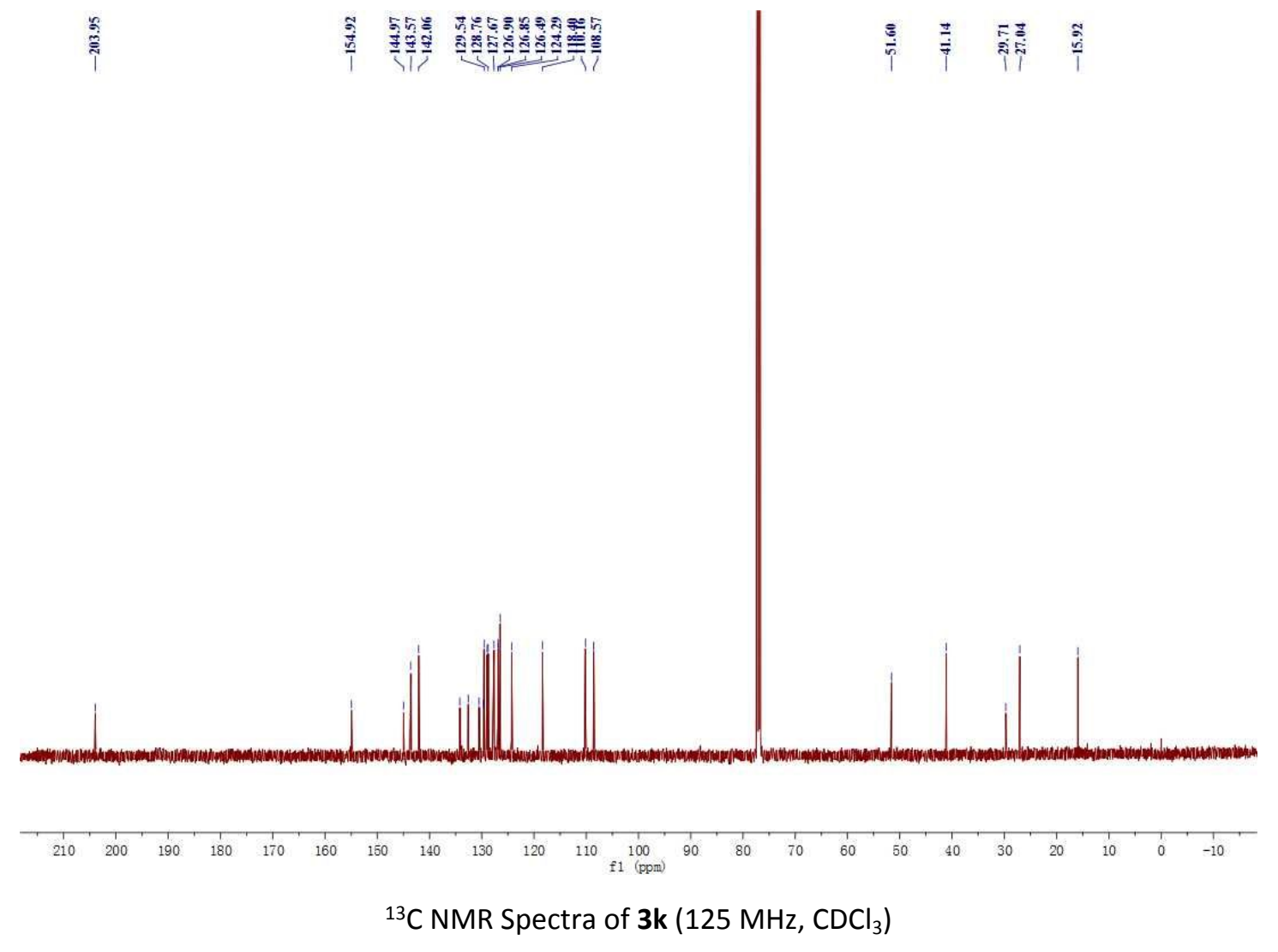




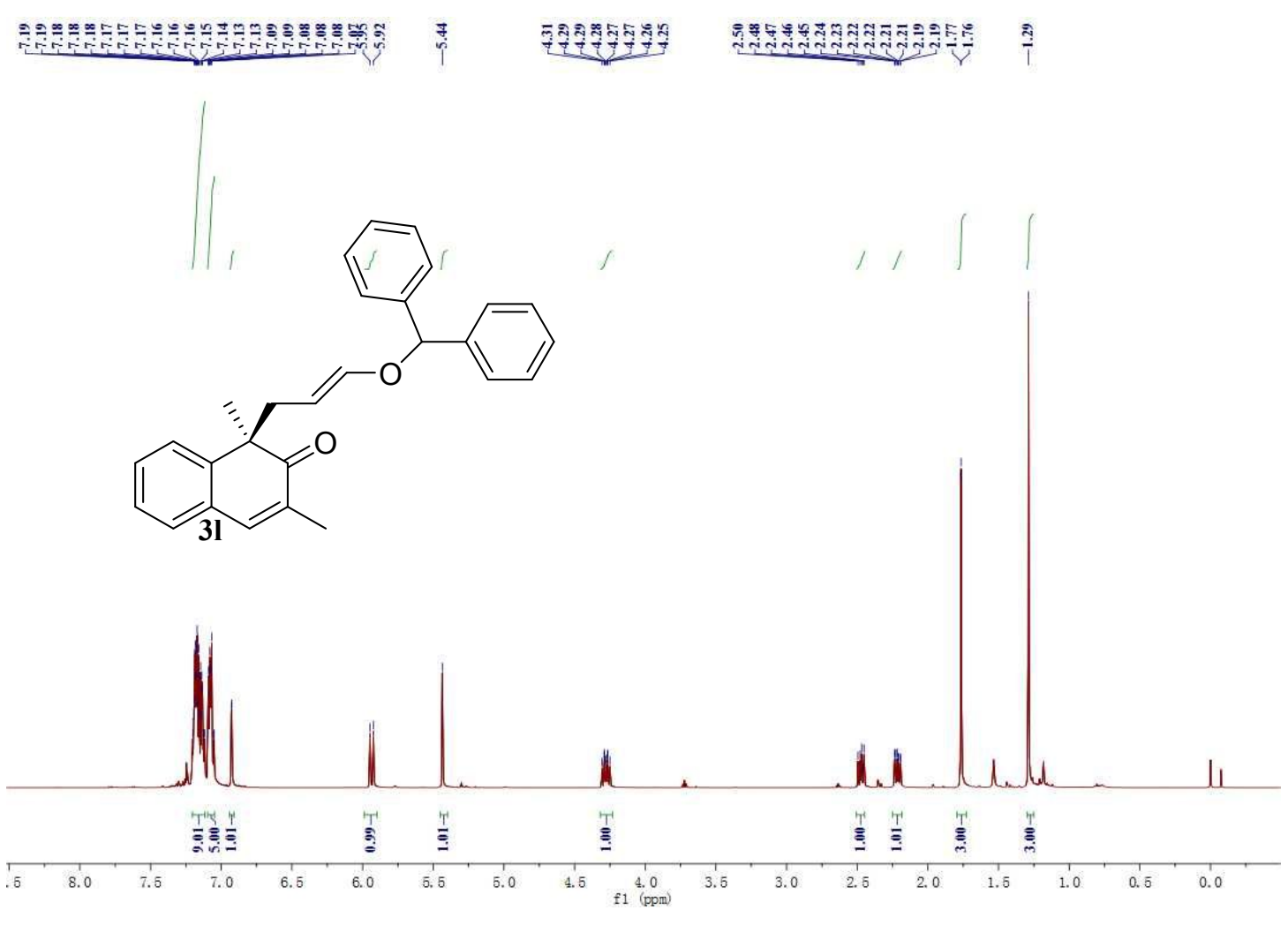

${ }^{1} \mathrm{H}$ NMR Spectra of $3 \mathbf{3}\left(500 \mathrm{MHz}, \mathrm{CDCl}_{3}\right)$

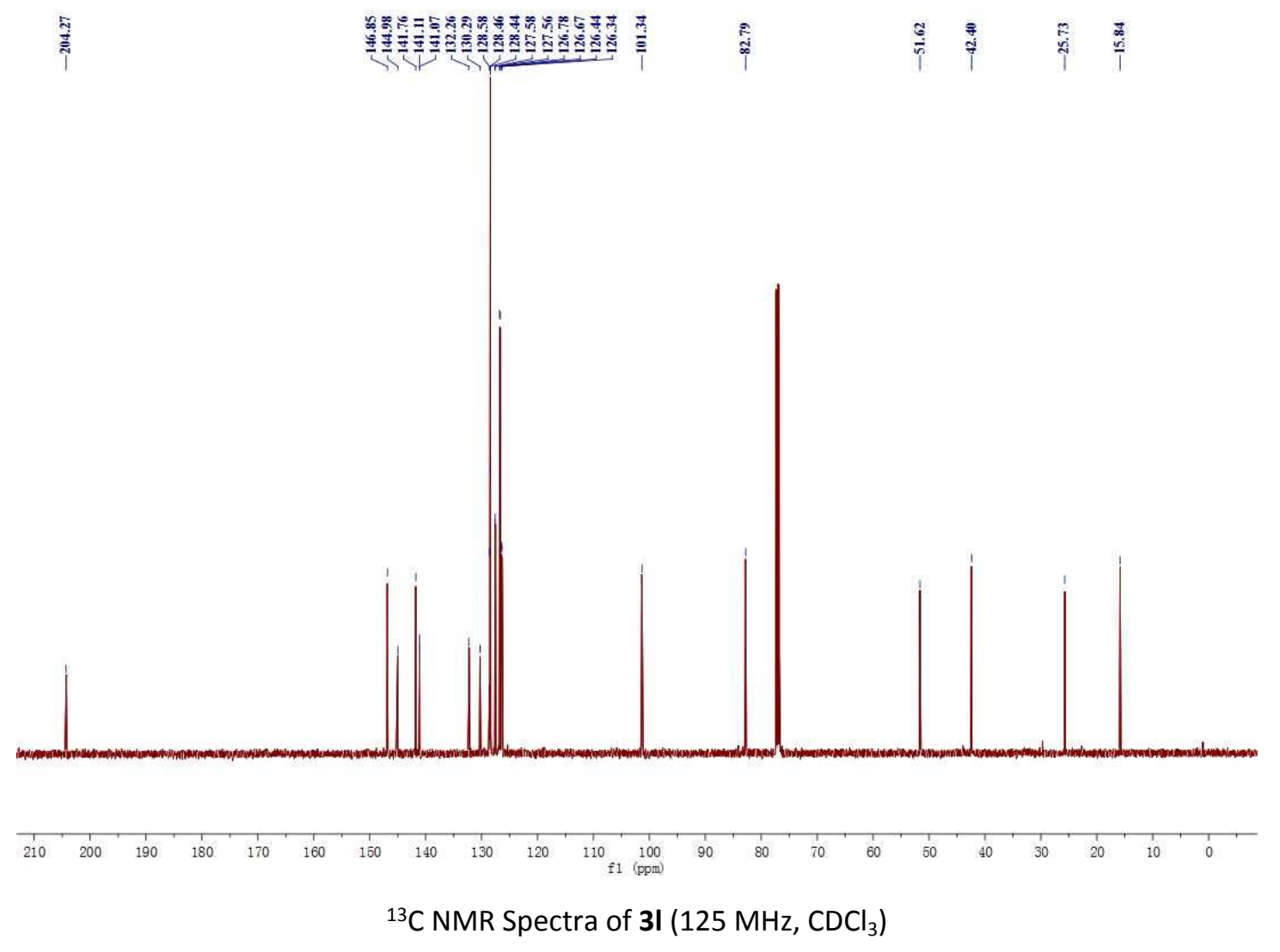




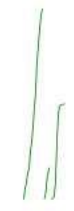<smiles>CC[C@]1(C/C=C/OCc2ccccc2)C(=O)C(C)=C[C@H]2c3ccccc3C21</smiles>

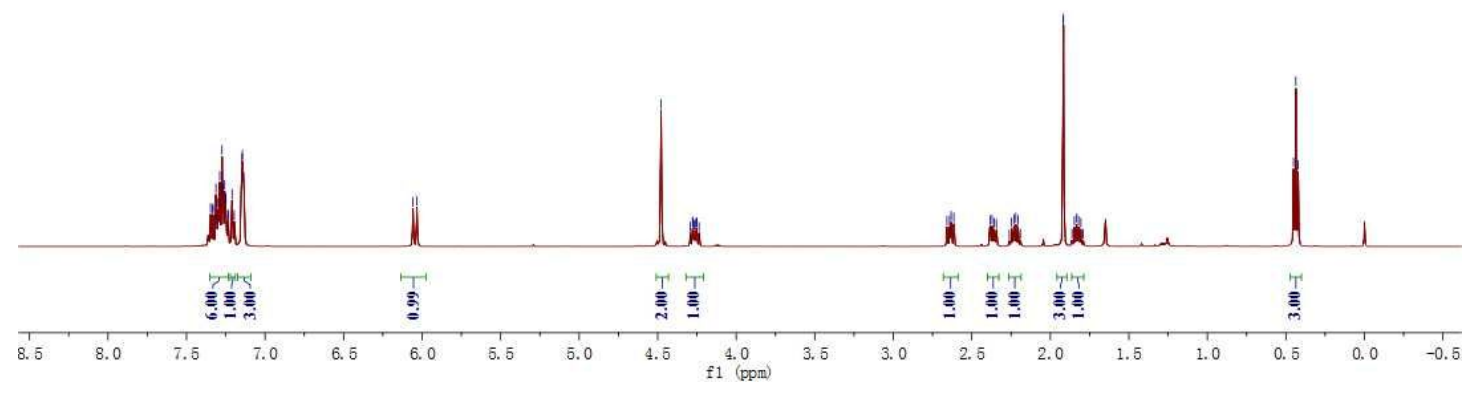

${ }^{1} \mathrm{H}$ NMR Spectra of $3 \mathrm{~m}\left(500 \mathrm{MHz}, \mathrm{CDCl}_{3}\right)$
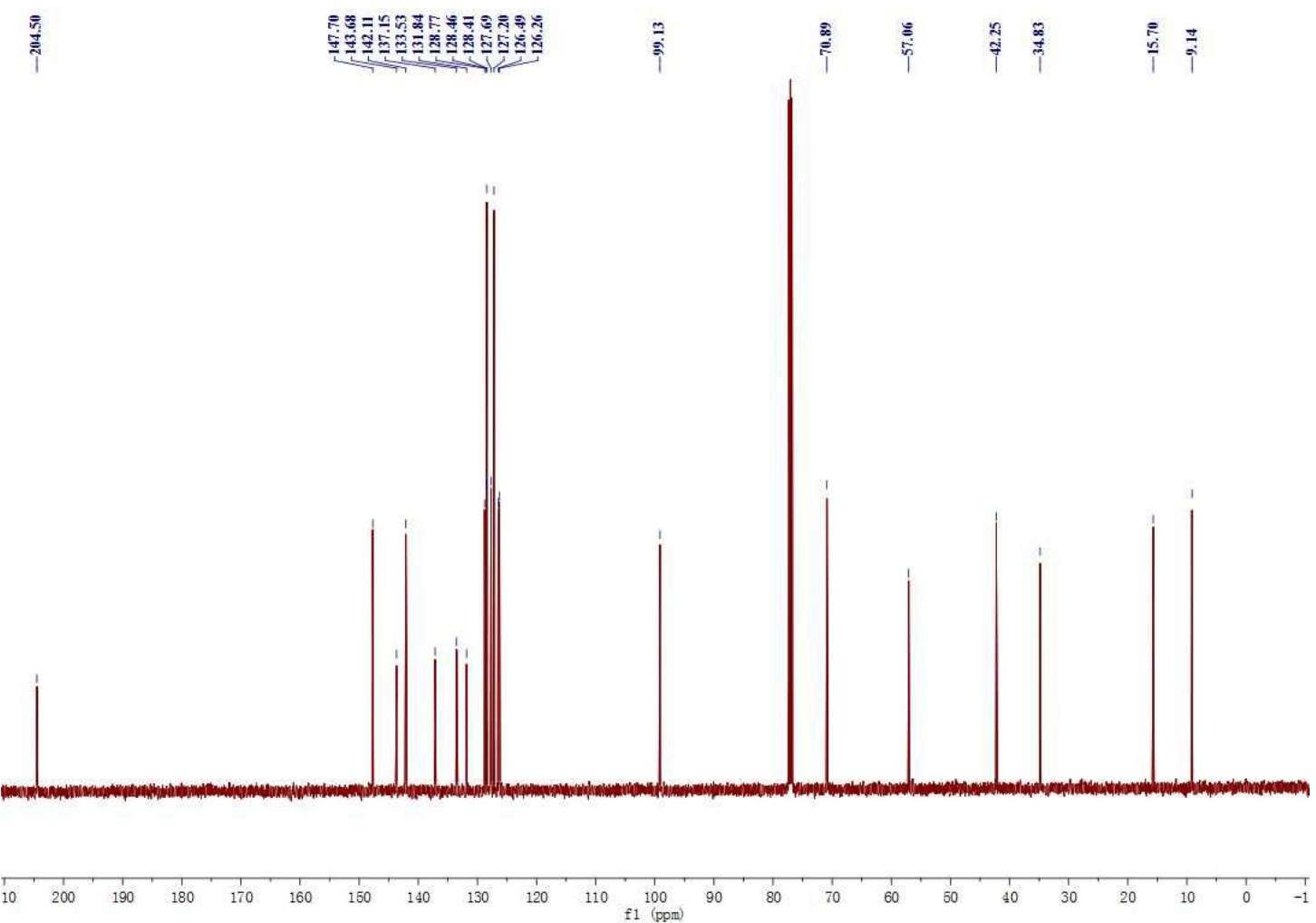

${ }^{13} \mathrm{C}$ NMR Spectra of $3 \mathrm{~m}\left(125 \mathrm{MHz}, \mathrm{CDCl}_{3}\right)$ 


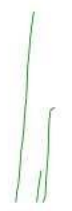<smiles></smiles>

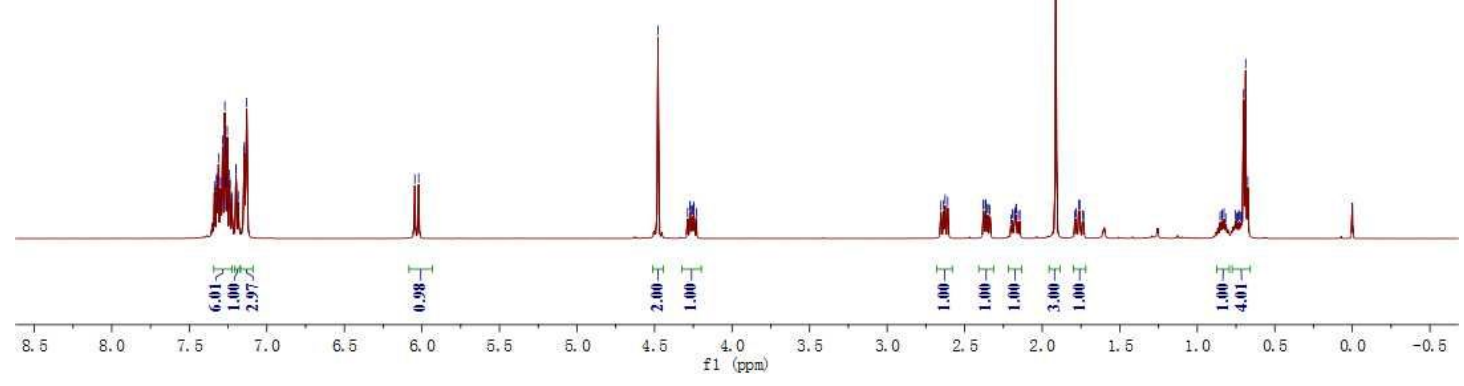

${ }^{1} \mathrm{H}$ NMR Spectra of $3 \mathbf{n}\left(500 \mathrm{MHz}, \mathrm{CDCl}_{3}\right)$

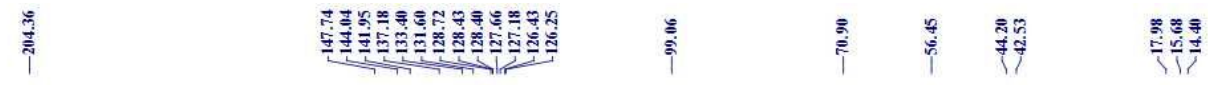
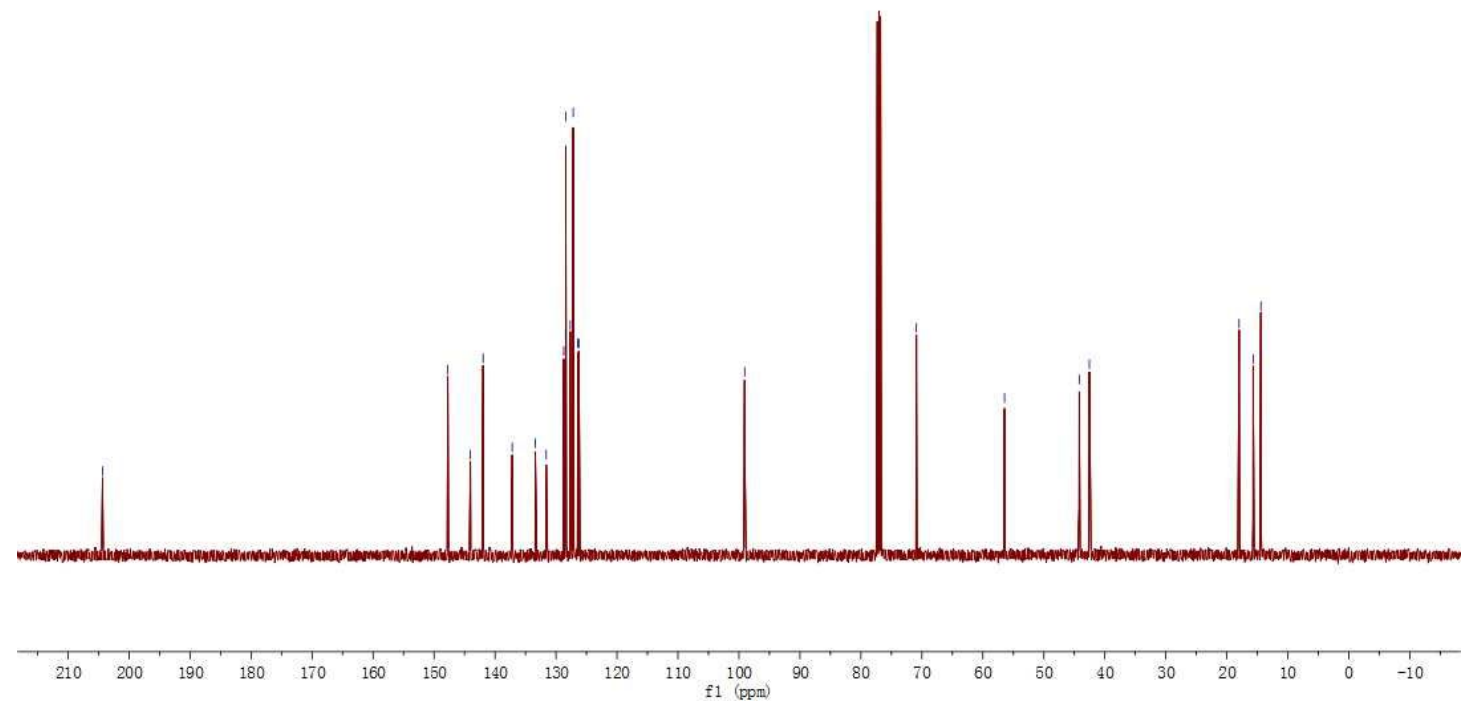

${ }^{13} \mathrm{C}$ NMR Spectra of $3 \mathbf{n}\left(125 \mathrm{MHz}, \mathrm{CDCl}_{3}\right)$ 
<smiles>CC#CC</smiles><smiles>CCCC[C@]1(C/C=C/OCc2ccccc2)C(=O)C(C)=Cc2ccccc21</smiles><smiles>C1CC2CC1C2</smiles><smiles>CC(C)C1C=CC1C</smiles>

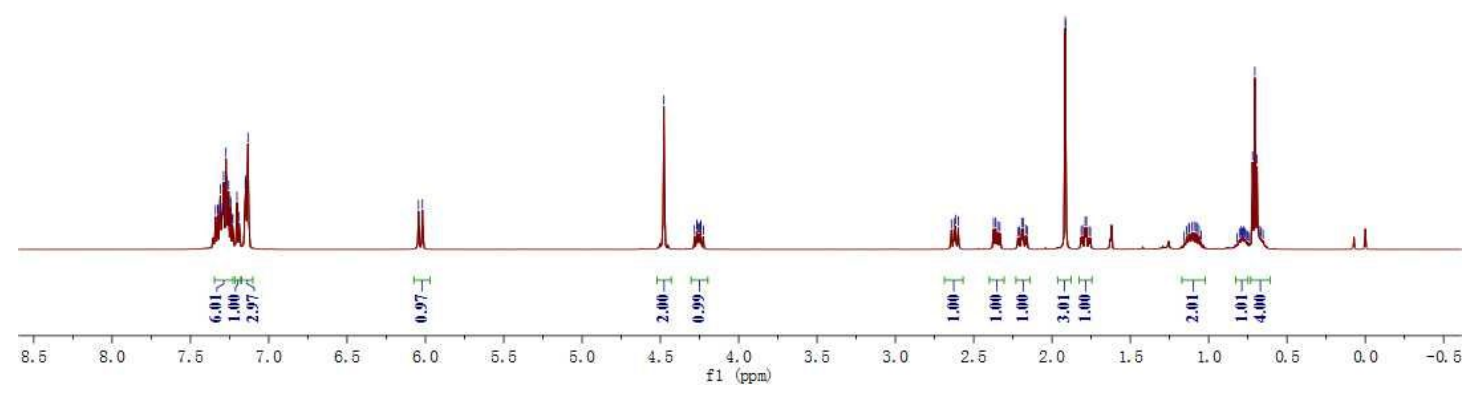

${ }^{1} \mathrm{H}$ NMR Spectra of $30\left(500 \mathrm{MHz}, \mathrm{CDCl}_{3}\right)$

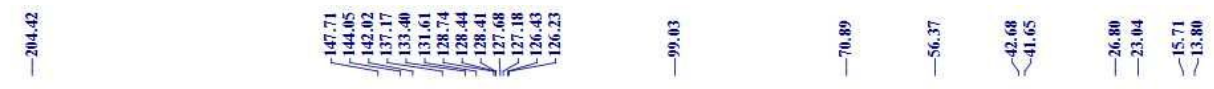
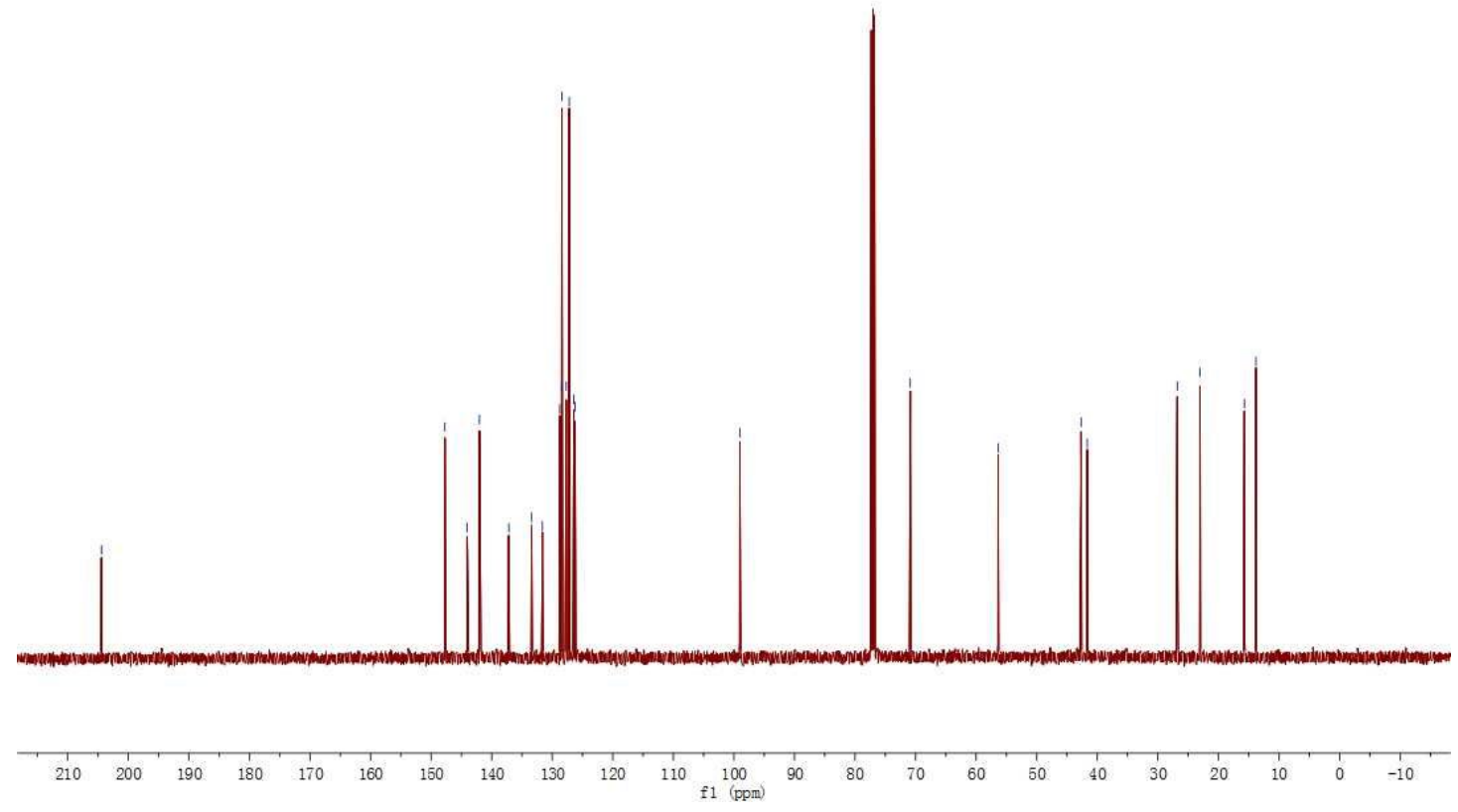

${ }^{13} \mathrm{C}$ NMR Spectra of $30\left(125 \mathrm{MHz}, \mathrm{CDCl}_{3}\right)$ 

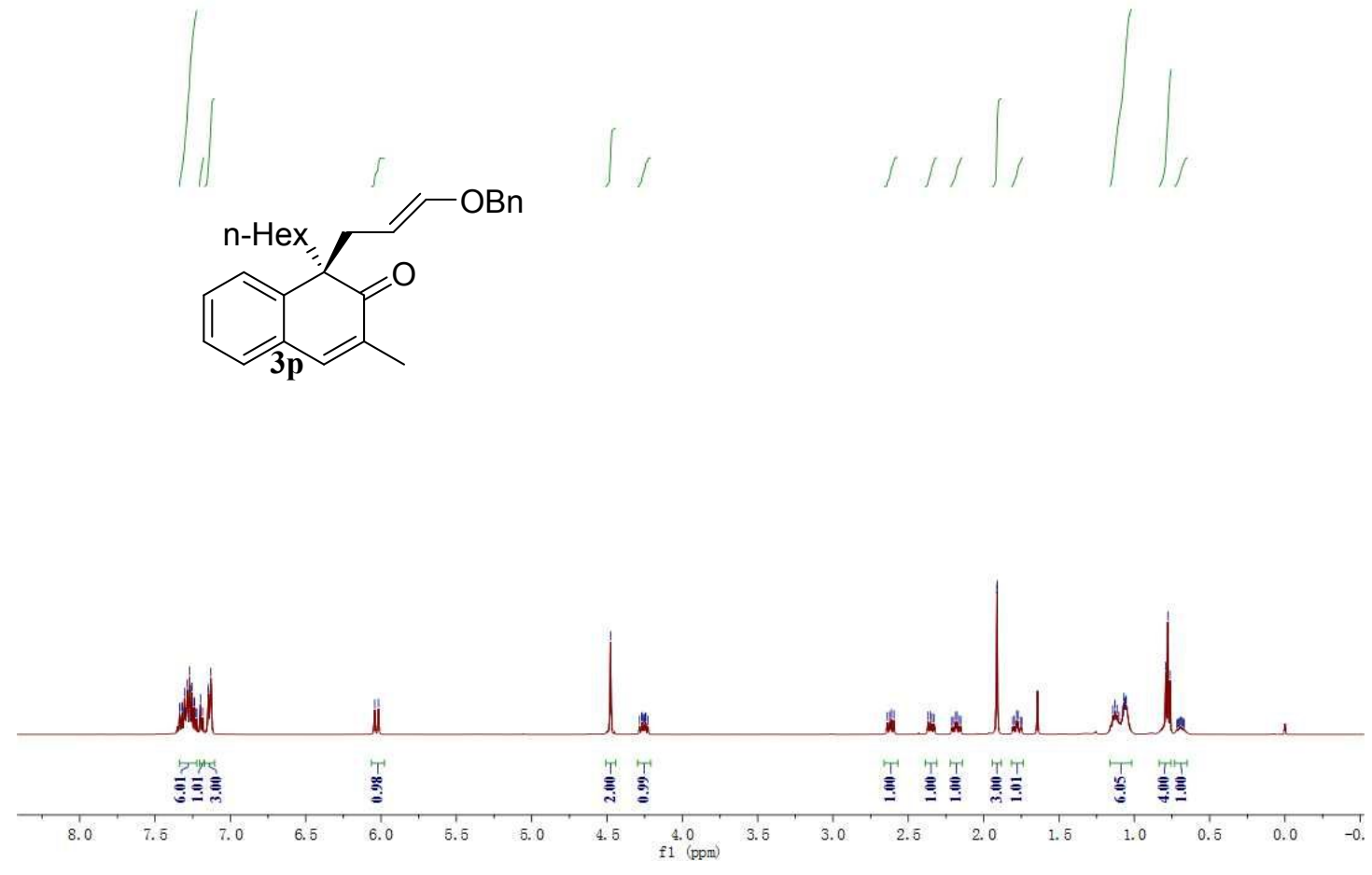

${ }^{1} \mathrm{H}$ NMR Spectra of $3 p\left(500 \mathrm{MHz}, \mathrm{CDCl}_{3}\right)$

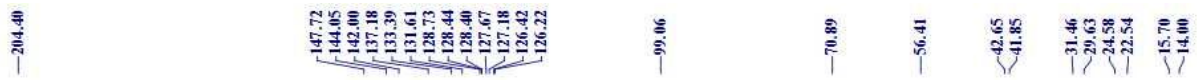
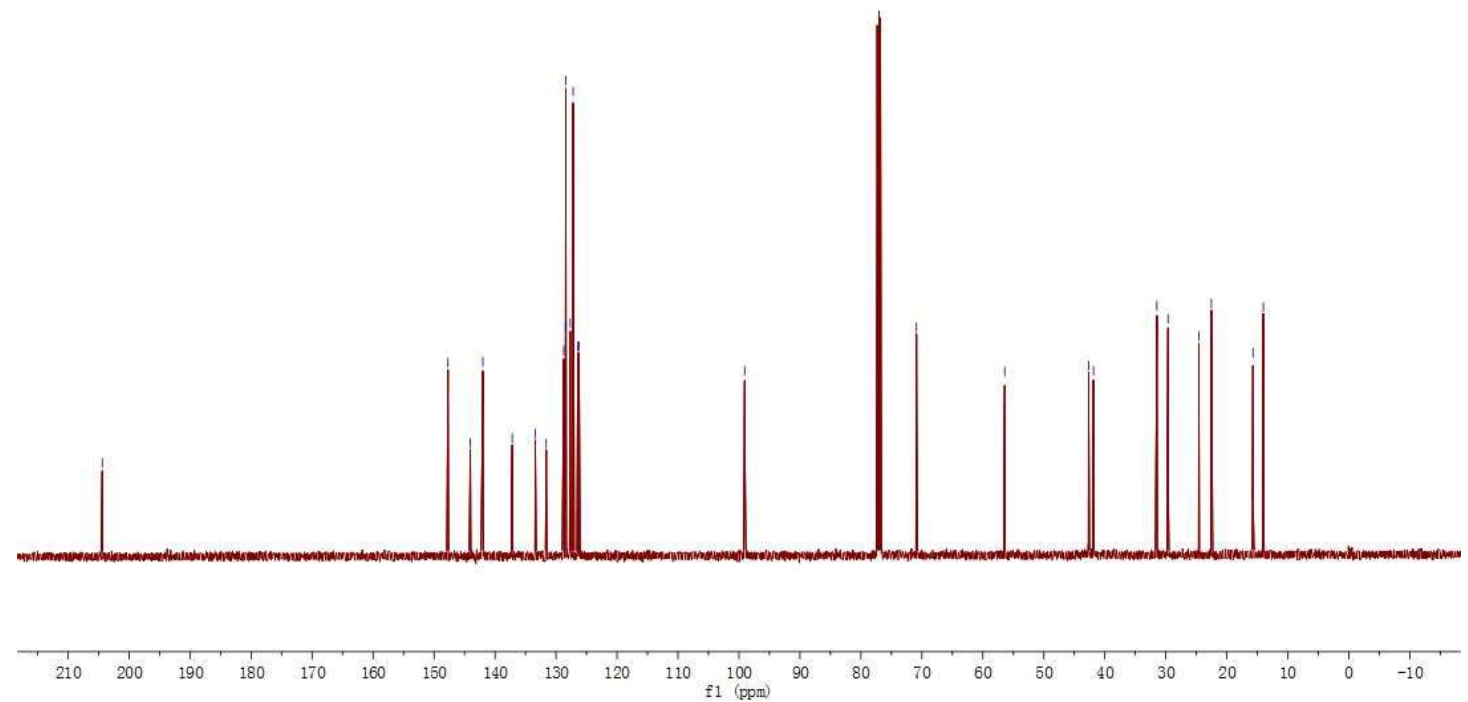

${ }^{13} \mathrm{C}$ NMR Spectra of $3 p\left(125 \mathrm{MHz}, \mathrm{CDCl}_{3}\right)$ 
$\|$

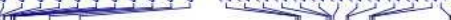<smiles>C=CC[C@]1(C/C=C/OCc2ccccc2)C(=O)C(C)=Cc2ccccc21</smiles>

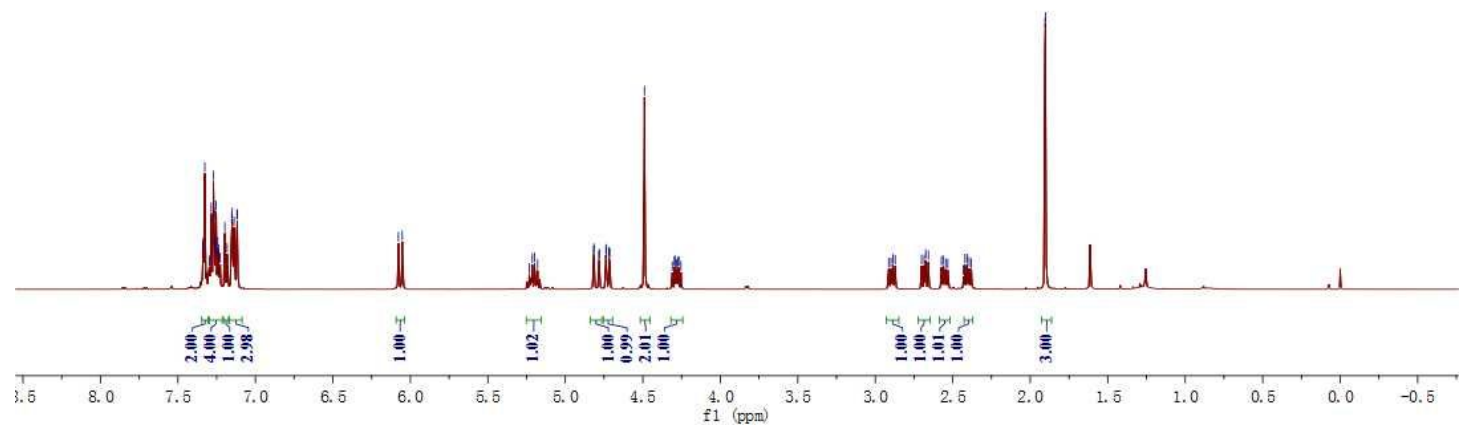

${ }^{1} \mathrm{H}$ NMR Spectra of $\mathbf{3 q}\left(500 \mathrm{MHz}, \mathrm{CDCl}_{3}\right)$

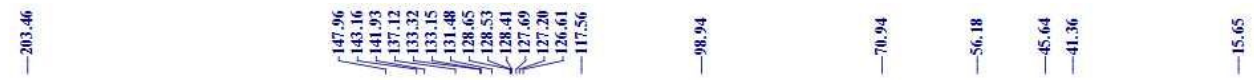
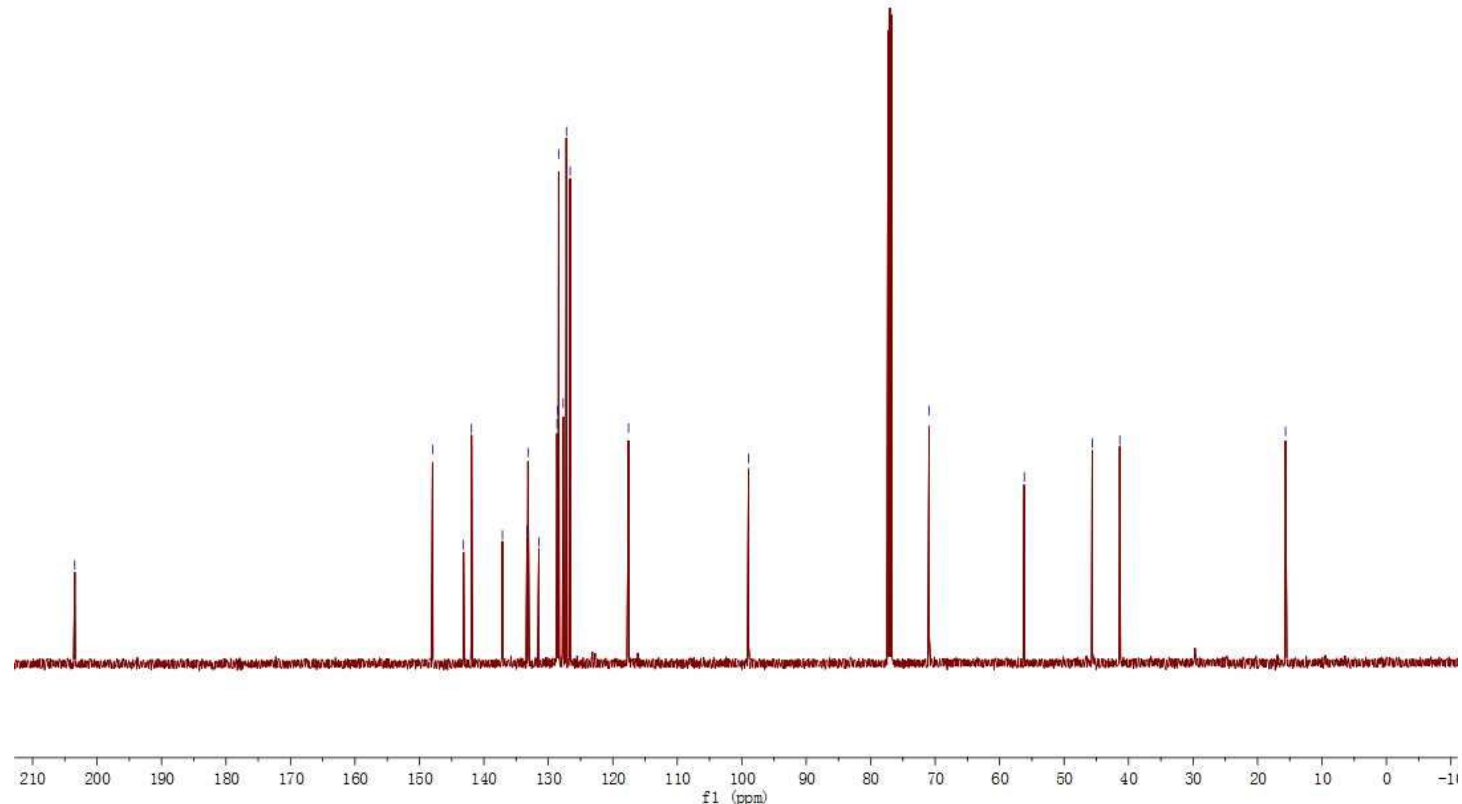

${ }^{13} \mathrm{C}$ NMR Spectra of $\mathbf{3 q}\left(125 \mathrm{MHz}, \mathrm{CDCl}_{3}\right)$ 
<smiles>CCC1=Cc2ccccc2[C@@](C)(C/C=C/OCc2ccccc2)C1=O</smiles>

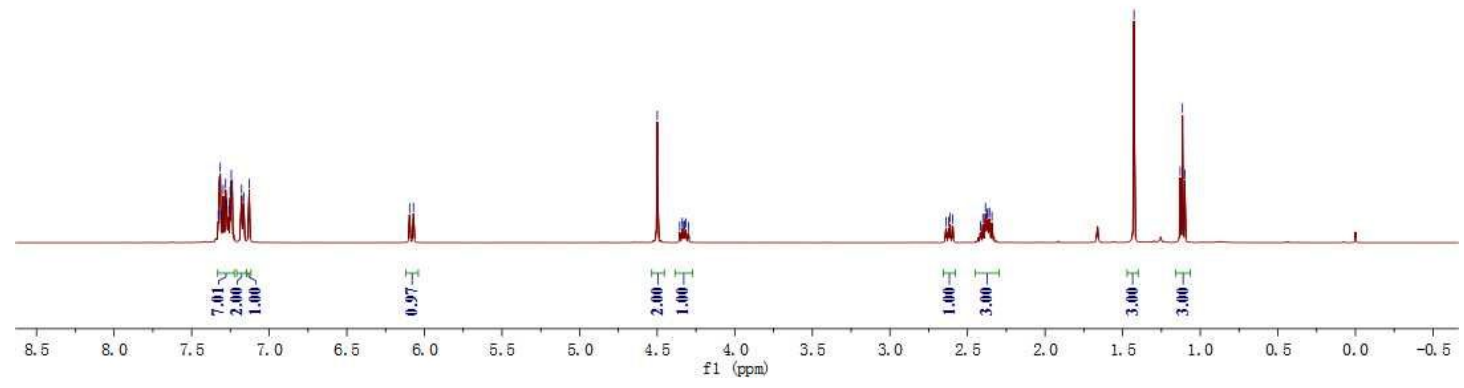
${ }^{1} \mathrm{H}$ NMR Spectra of $3 r\left(500 \mathrm{MHz}, \mathrm{CDCl}_{3}\right)$

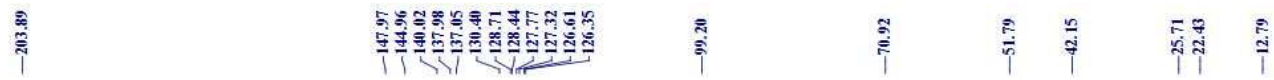
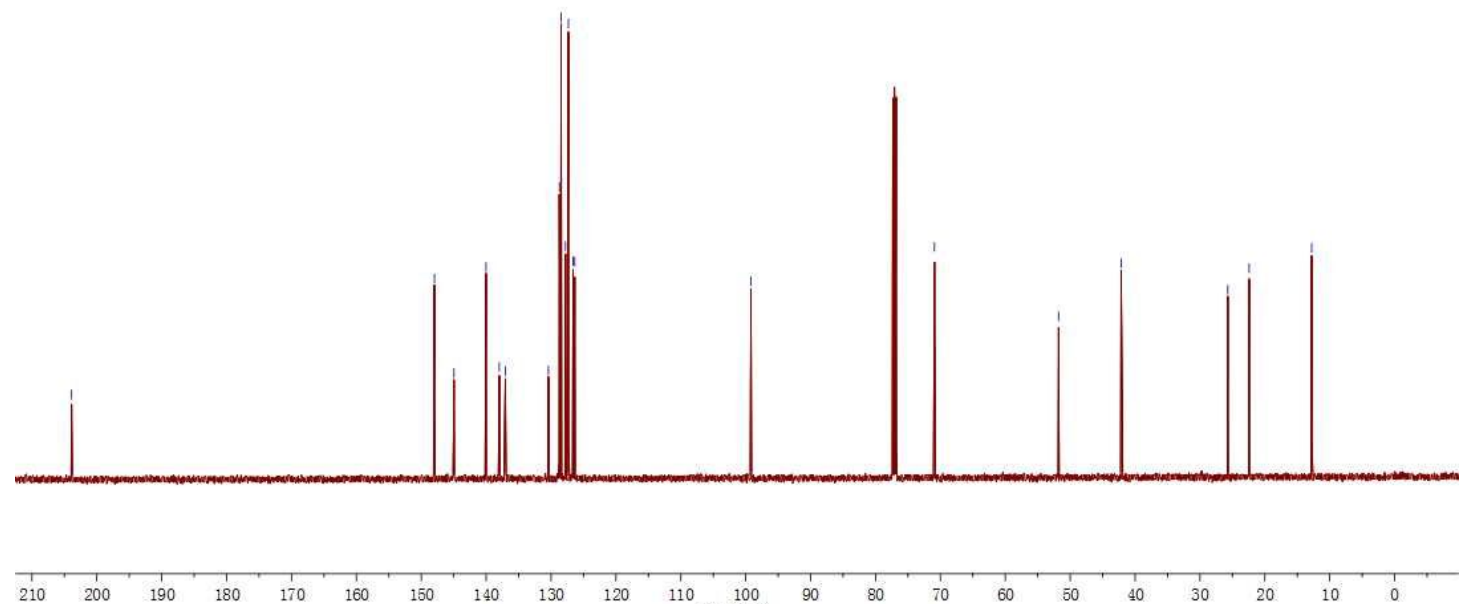

${ }^{13} \mathrm{C}$ NMR Spectra of $3 r\left(125 \mathrm{MHz}, \mathrm{CDCl}_{3}\right)$ 


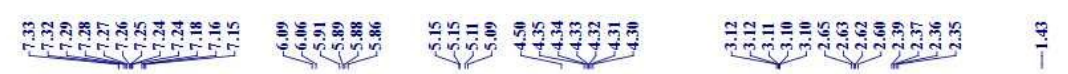

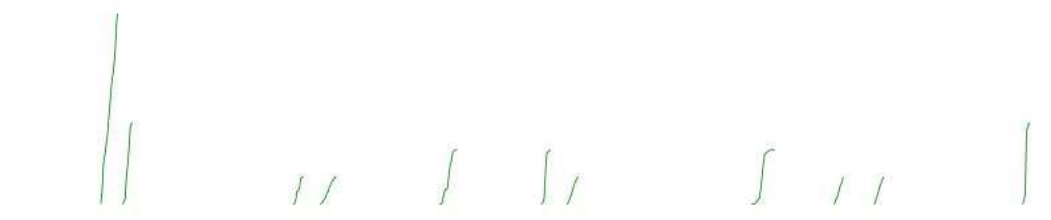<smiles>C=CCC1=Cc2ccccc2[C@@](C)(C/C=C/OCc2ccccc2)C1=O</smiles>

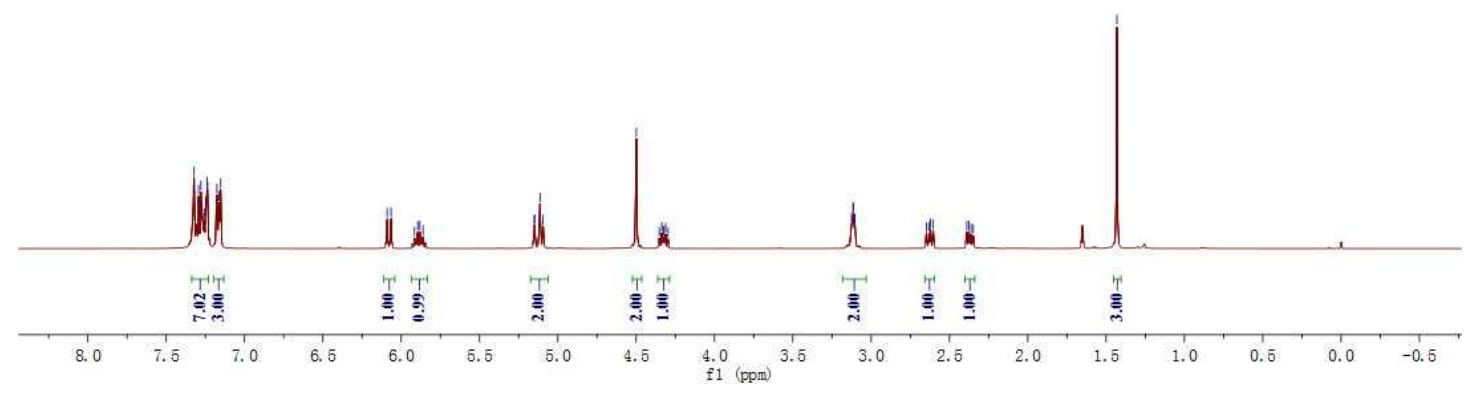

${ }^{1} \mathrm{H}$ NMR Spectra of $3 s\left(500 \mathrm{MHz}, \mathrm{CDCl}_{3}\right)$

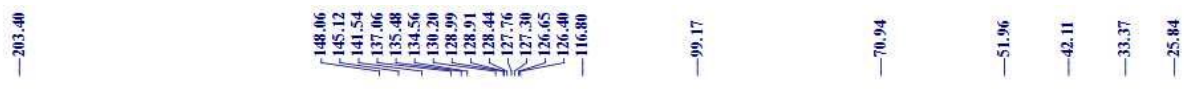
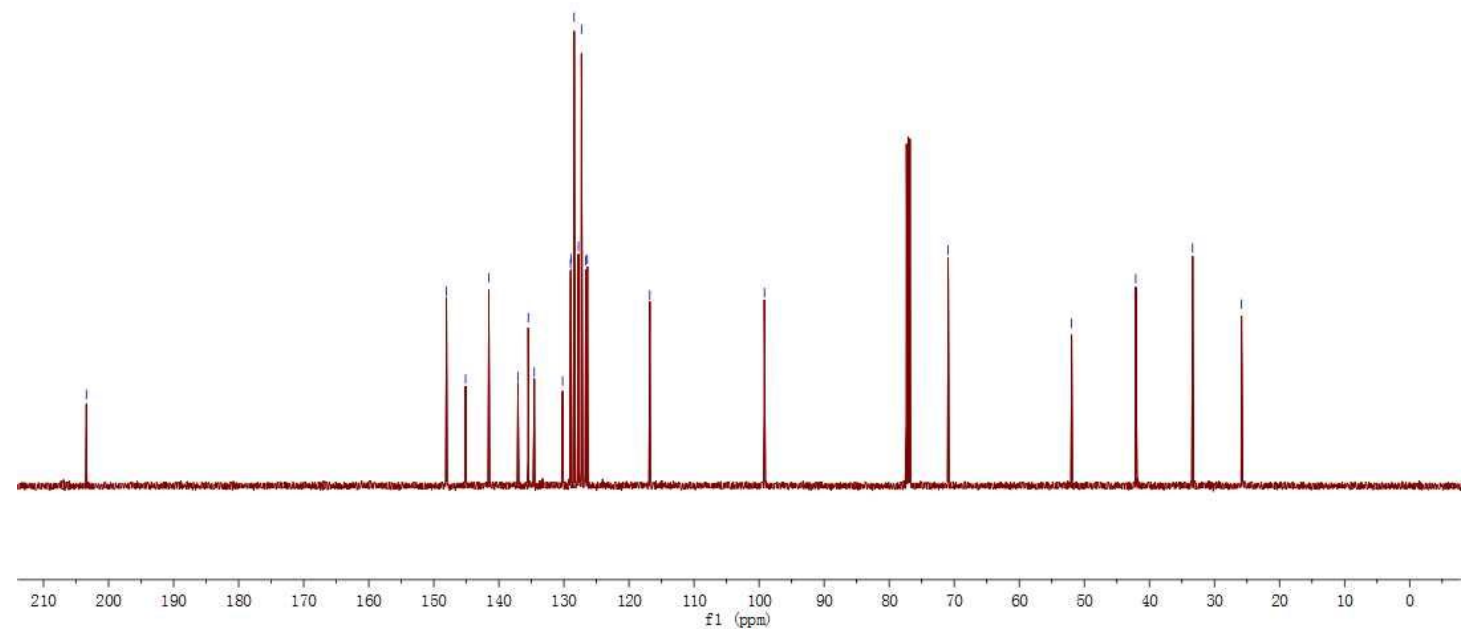

${ }^{13} \mathrm{C}$ NMR Spectra of $3 s\left(125 \mathrm{MHz}, \mathrm{CDCl}_{3}\right)$ 


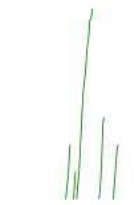<smiles>C[C@]1(C/C=C/OCc2ccccc2)C(=O)C(c2ccccc2)=Cc2ccccc21</smiles>

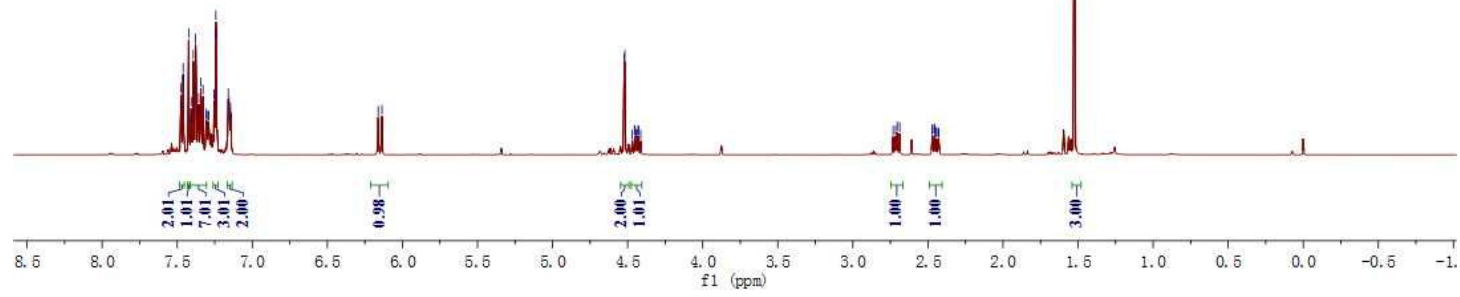

${ }^{1} \mathrm{H}$ NMR Spectra of $3 \mathbf{t}\left(500 \mathrm{MHz}, \mathrm{CDCl}_{3}\right)$

จิ
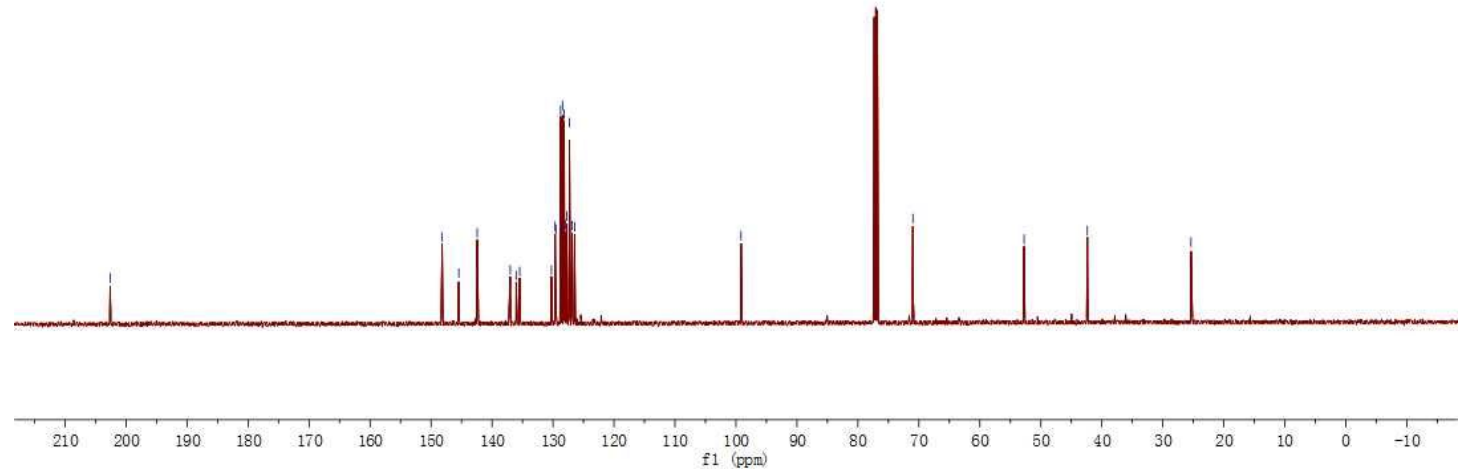

${ }^{13} \mathrm{C}$ NMR Spectra of $3 \mathbf{t}\left(125 \mathrm{MHz}, \mathrm{CDCl}_{3}\right)$ 

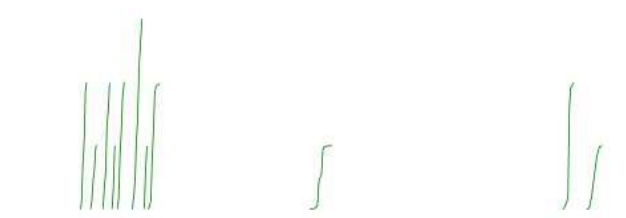<smiles>CC1=C[C@@H]2C=C(c3ccccc3)C=C[C@]2(C)C(=O)C1=CC=COCc1ccccc1</smiles>

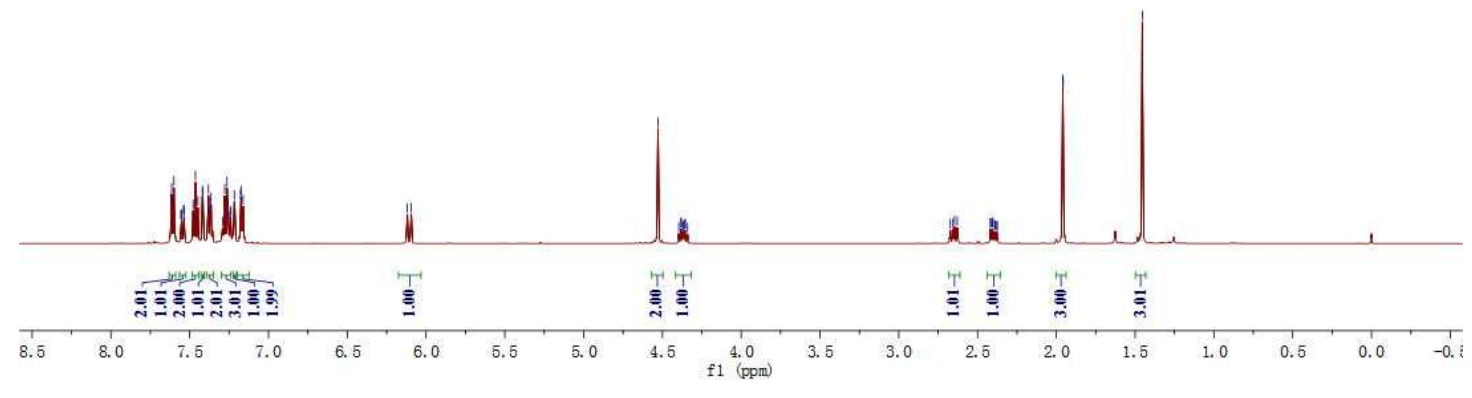

${ }^{1} \mathrm{H}$ NMR Spectra of $\mathbf{3 u}\left(500 \mathrm{MHz}, \mathrm{CDCl}_{3}\right)$

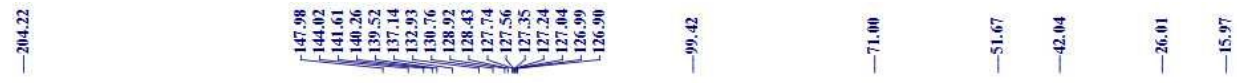

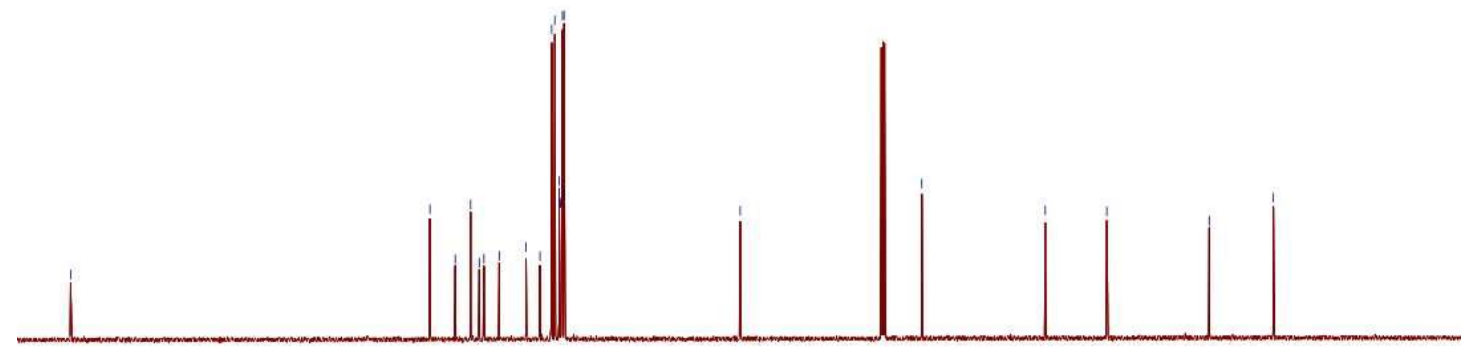

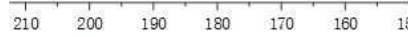
$\mathrm{f1} \stackrel{100}{(\mathrm{ppm})}$

${ }^{13} \mathrm{C}$ NMR Spectra of $3 \mathbf{u}\left(125 \mathrm{MHz}, \mathrm{CDCl}_{3}\right)$ 


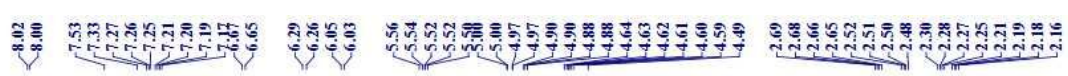

i 1<smiles>C=CC[C@]1(C/C=C/OCc2ccccc2)C=C[C@H]2C=CC=CC21C</smiles>

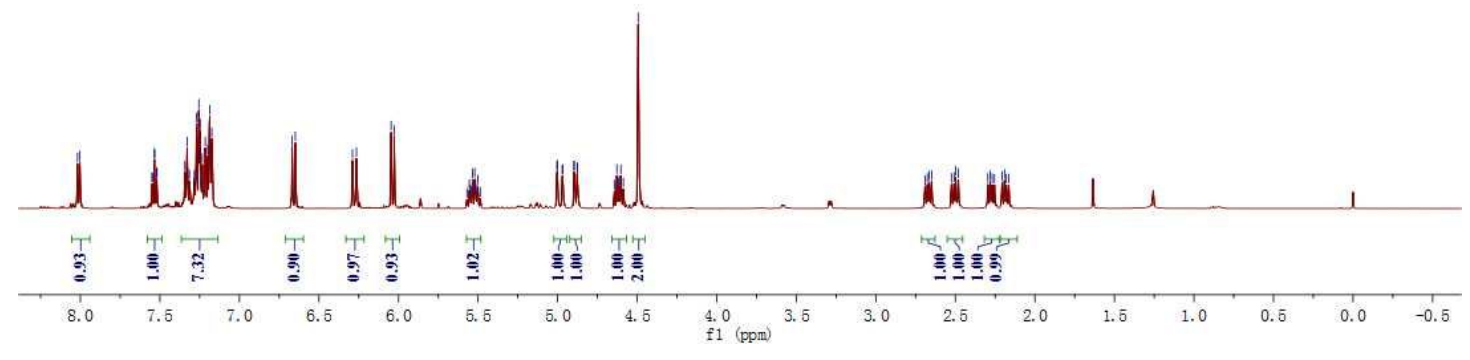

${ }^{1} \mathrm{H}$ NMR Spectra of $5 a\left(500 \mathrm{MHz}, \mathrm{CDCl}_{3}\right)$

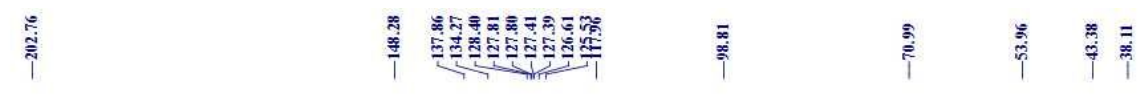

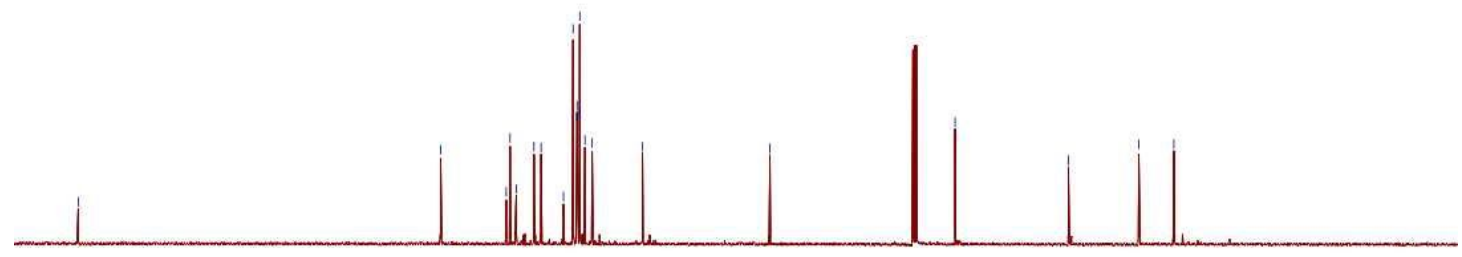

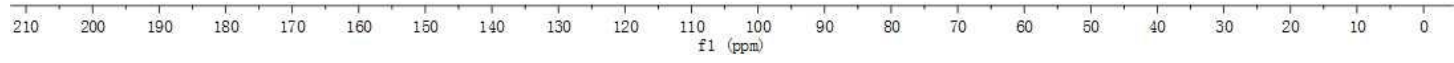

${ }^{13} \mathrm{C}$ NMR Spectra of $5 \mathrm{a}\left(125 \mathrm{MHz}, \mathrm{CDCl}_{3}\right)$ 


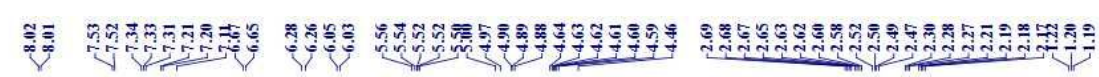

$\left.\left.\int 1 / 1\right) \int 1 / 1\right)$<smiles>C=CC[C@]1(C/C=C/OCc2ccc(CC)cc2)C=C[C@H]2C=CC=CC2C1=O</smiles>

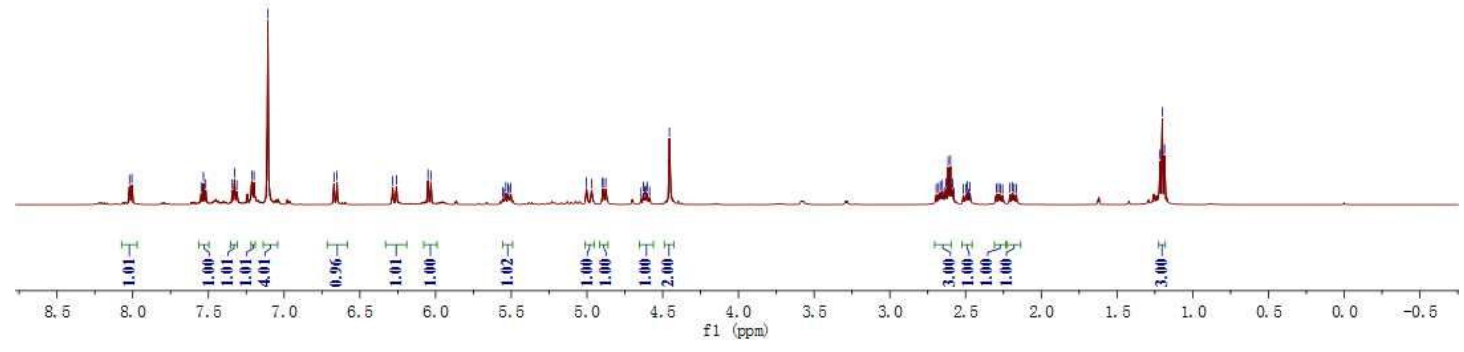

${ }^{1} \mathrm{H}$ NMR Spectra of $\mathbf{5 b}\left(500 \mathrm{MHz}, \mathrm{CDCl}_{3}\right)$
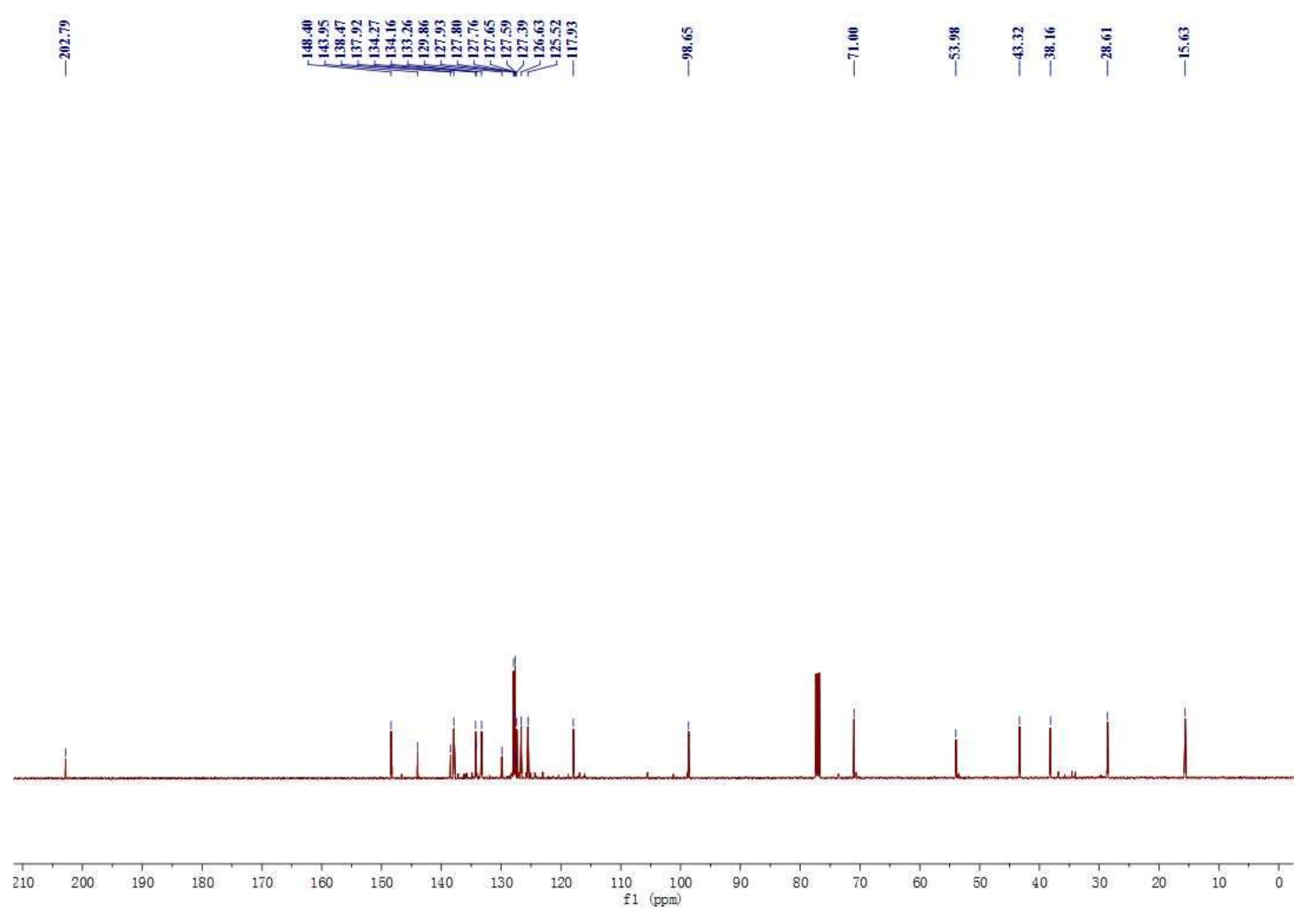

${ }^{13} \mathrm{C}$ NMR Spectra of $\mathbf{5 b}\left(125 \mathrm{MHz}, \mathrm{CDCl}_{3}\right)$ 


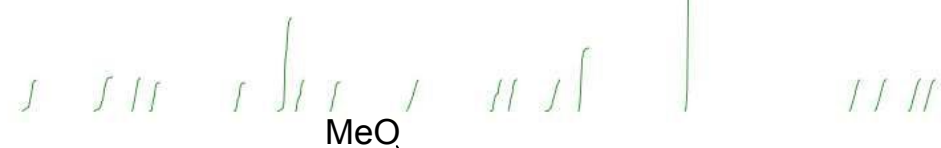<smiles>C=CC[C@]1(C/C=C/OCc2cc(OC)cc(OC)c2)C=C[C@H]2C=CC=C1C2=O</smiles>

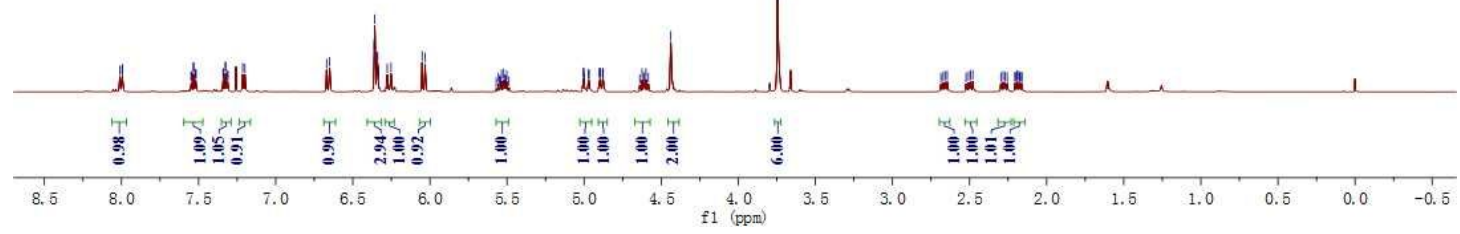

${ }^{1} \mathrm{H}$ NMR Spectra of $\mathbf{5 c}\left(500 \mathrm{MHz}, \mathrm{CDCl}_{3}\right)$

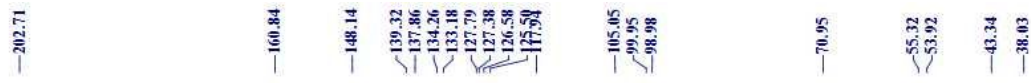
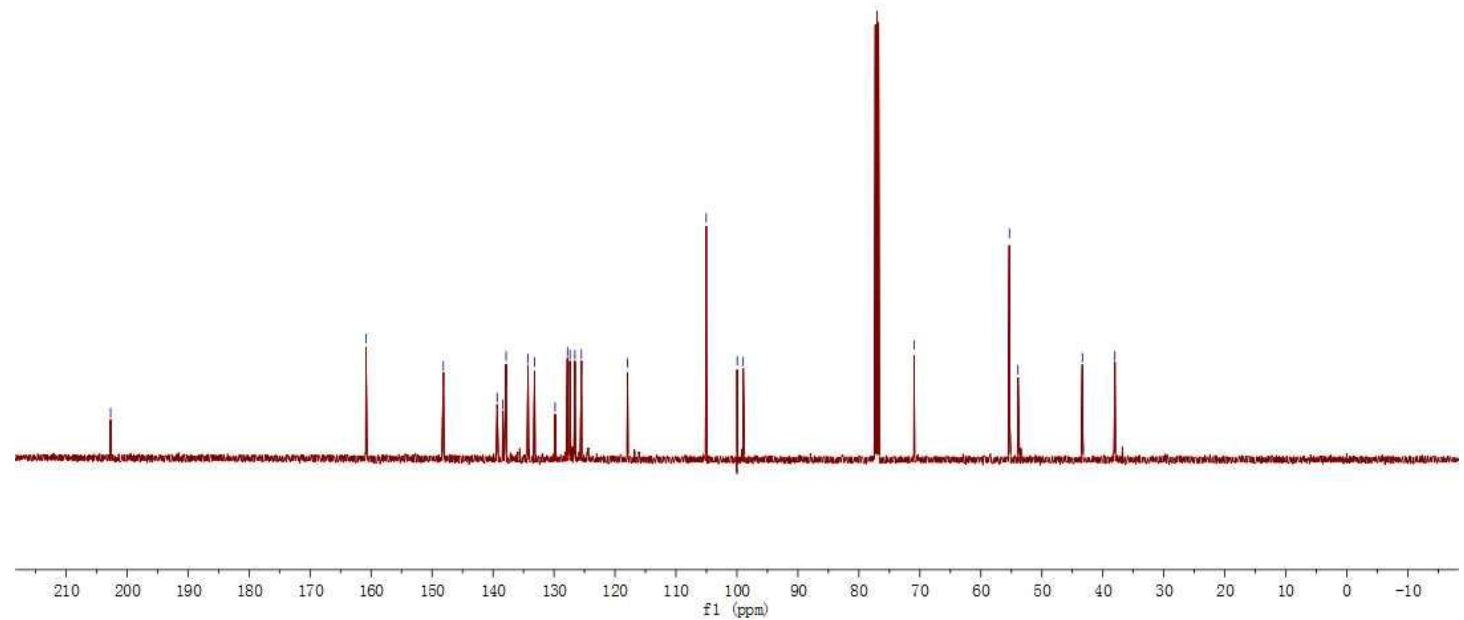

${ }^{13} \mathrm{C}$ NMR Spectra of $\mathbf{5 c}\left(125 \mathrm{MHz}, \mathrm{CDCl}_{3}\right)$ 


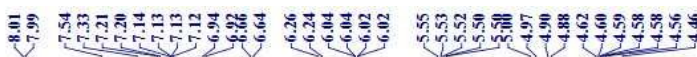

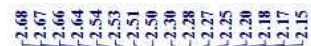
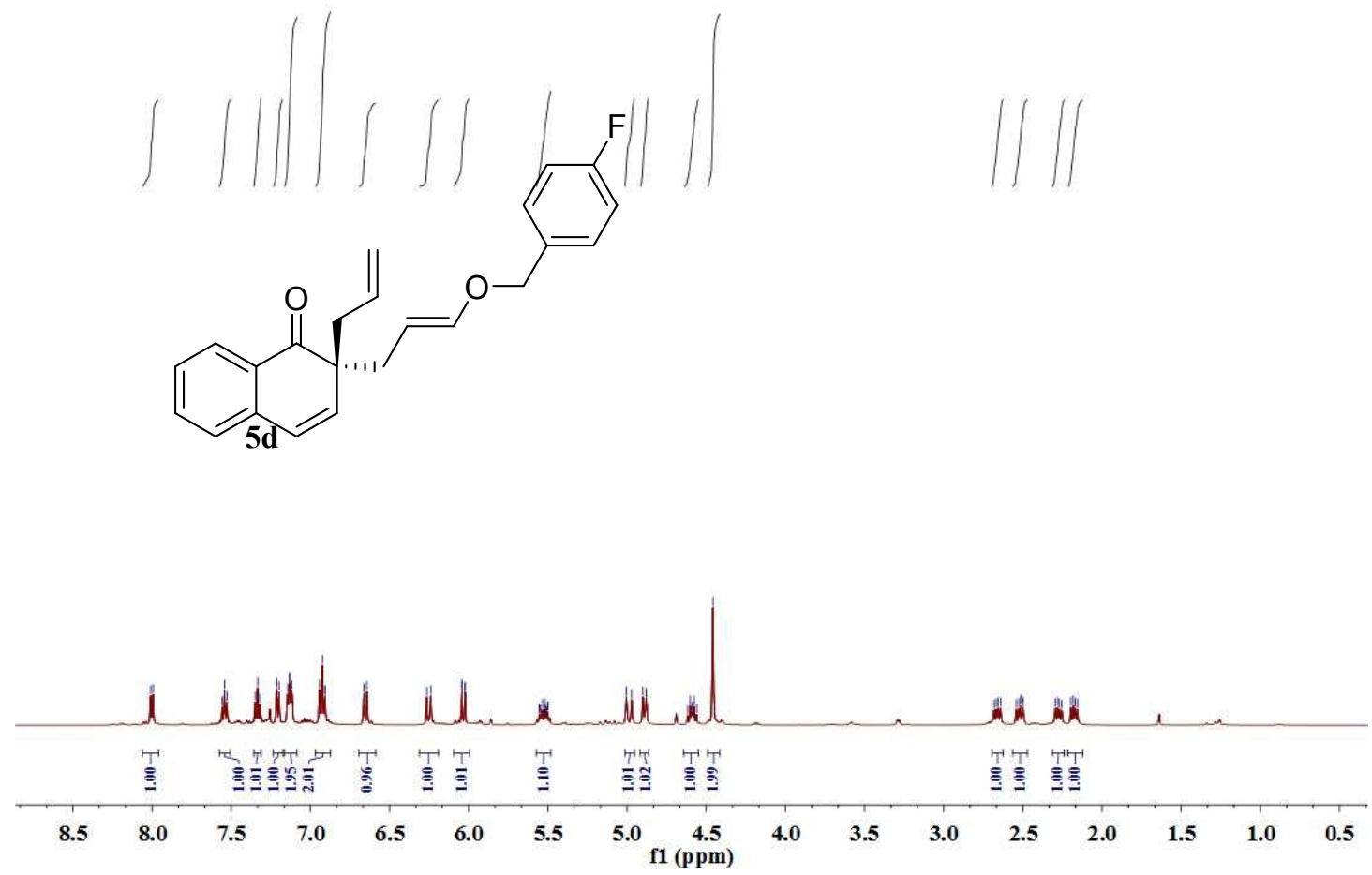

${ }^{1} \mathrm{H}$ NMR Spectra of $\mathbf{5 d}\left(500 \mathrm{MHz}, \mathrm{CDCl}_{3}\right)$

₹

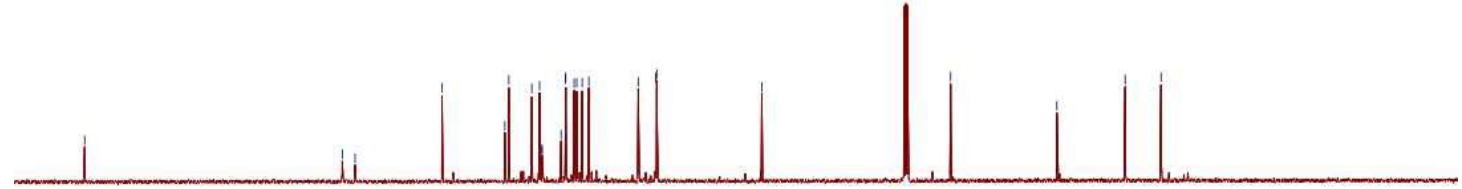

$\begin{array}{llllllllllllllllllllll}210 & 200 & 190 & 180 & 170 & 160 & 150 & 140 & 130 & 120 & 110 & 100 & 90 & 80 & 70 & 60 & 50 & 40 & 30 & 20 & 10 & 0\end{array}$

${ }^{13} \mathrm{C}$ NMR Spectra of $\mathbf{5} \mathbf{d}\left(125 \mathrm{MHz}, \mathrm{CDCl}_{3}\right)$ 


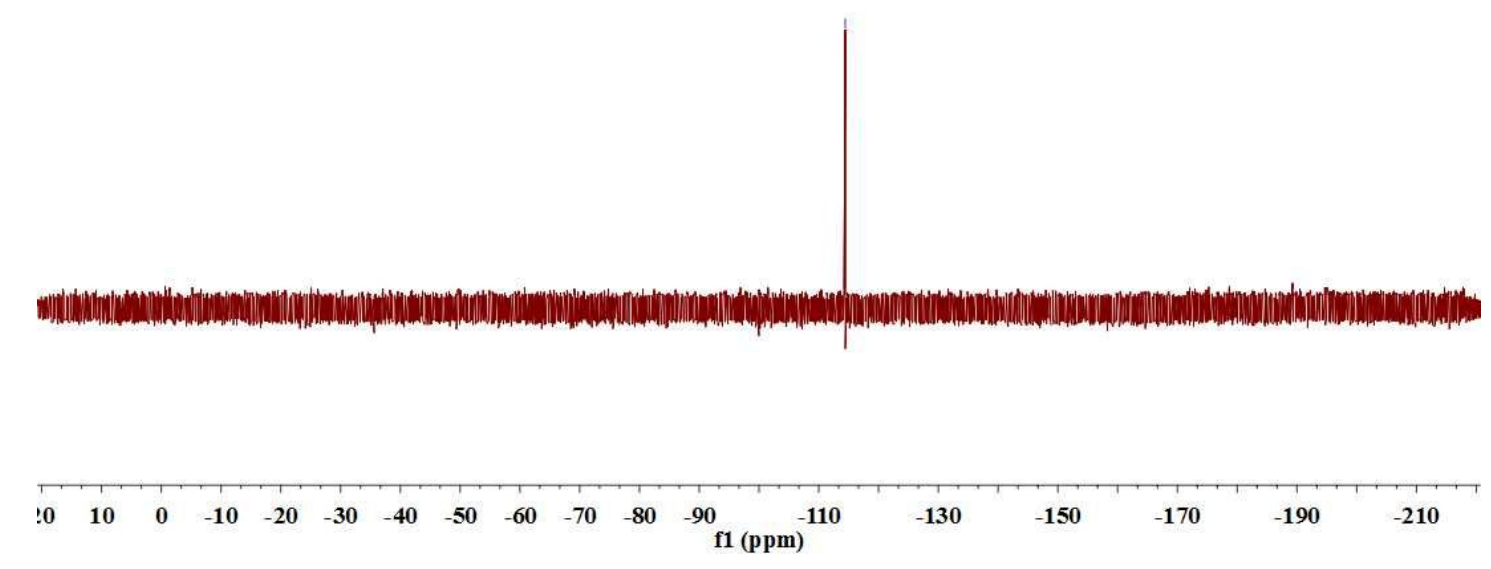

${ }^{19} \mathrm{~F}$ NMR Spectra of $\mathbf{5 d}\left(470 \mathrm{MHz}, \mathrm{CDCl}_{3}\right)$

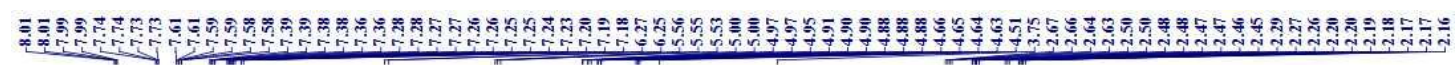

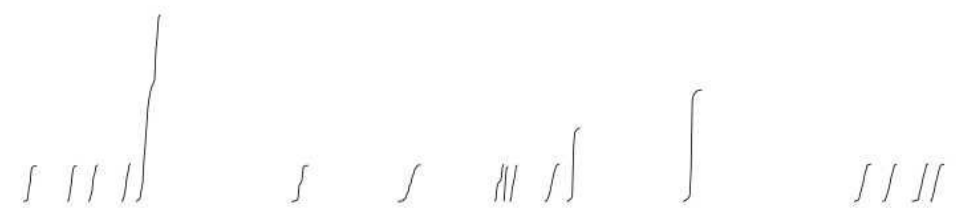<smiles>C=CC[C@]1(C/C=C/OCc2ccccc2)C=C(OC)c2ccccc2C1=O</smiles>

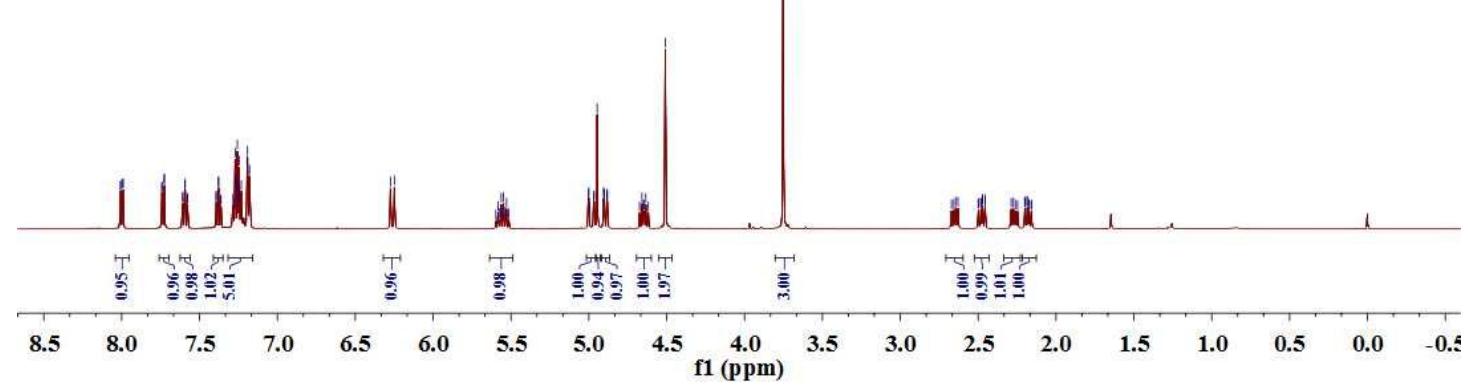

${ }^{1} \mathrm{H}$ NMR Spectra of $5 \mathbf{e}\left(500 \mathrm{MHz}, \mathrm{CDCl}_{3}\right)$ 


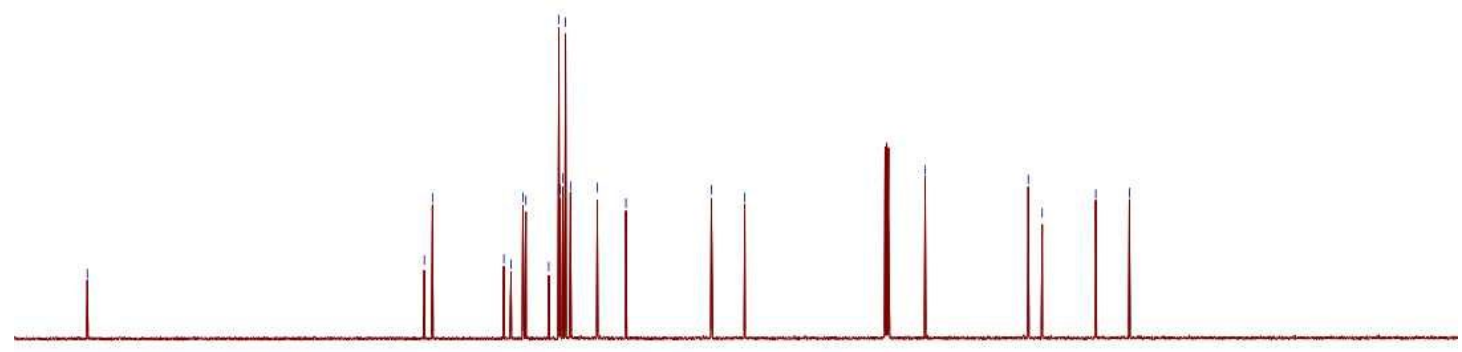

$\begin{array}{lllllllllllllllllllllll}210 & 200 & 190 & 180 & 170 & 160 & 150 & 140 & 130 & 120 & \begin{array}{c}110 \\ \mathrm{f} 1(\mathrm{ppm})\end{array} & \begin{array}{ll}100 \\ (\mathrm{ppm}\end{array} & 80 & 70 & 60 & 50 & 40 & 30 & 20 & 10 & 0 & -10\end{array}$ ${ }^{13} \mathrm{C}$ NMR Spectra of 5 e $\left(125 \mathrm{MHz}, \mathrm{CDCl}_{3}\right)$

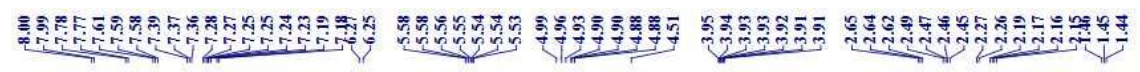

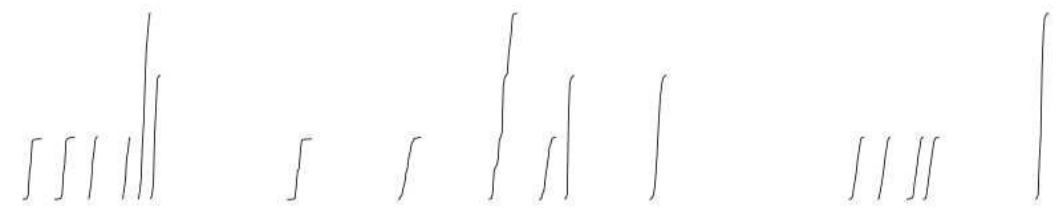<smiles>C=CC[C@]1(C/C=C/COCCCC)C=C(OCC)c2[c]cccc2C1=O</smiles>

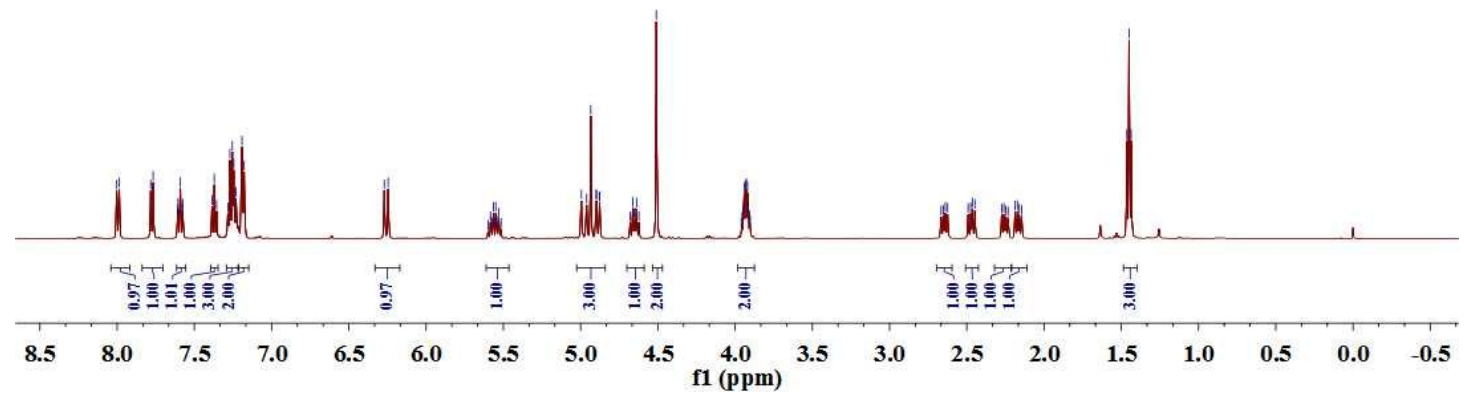

${ }^{1} \mathrm{H}$ NMR Spectra of $\mathbf{5 f}\left(500 \mathrm{MHz}, \mathrm{CDCl}_{3}\right)$ 


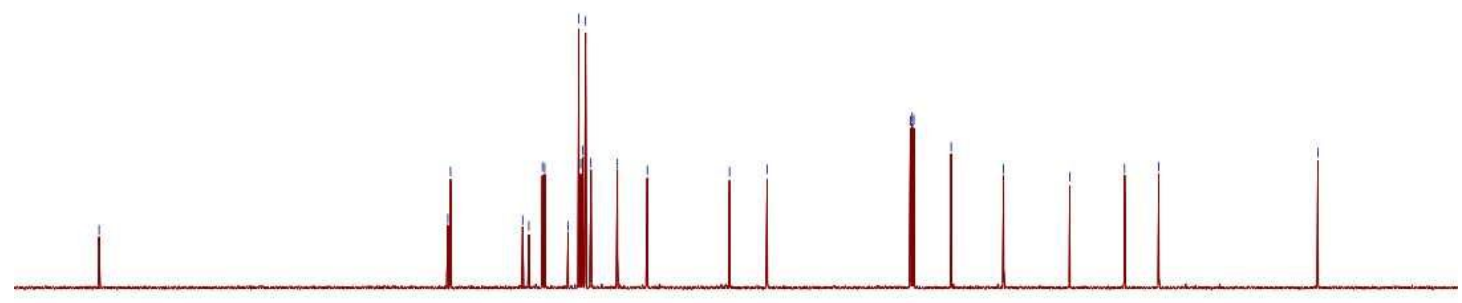

$\begin{array}{llllllllllllllllllllll}210 & 200 & 190 & 180 & 170 & 160 & 150 & 140 & 130 & 120 & \underset{110}{110} \begin{array}{c}100 \\ \text { f1 pm })\end{array} & 90 & 80 & 70 & 60 & 50 & 40 & 30 & 20 & 10 & 0\end{array}$

${ }^{13} \mathrm{C}$ NMR Spectra of $\mathbf{5} \mathbf{f}\left(125 \mathrm{MHz}, \mathrm{CDCl}_{3}\right)$

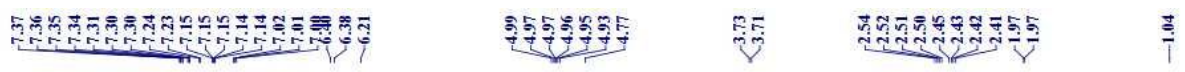<smiles>CC1=Cc2ccccc2[C@@](C)(CC/C=C/I=C/COc2ccccc2)[C@@H]1O</smiles>

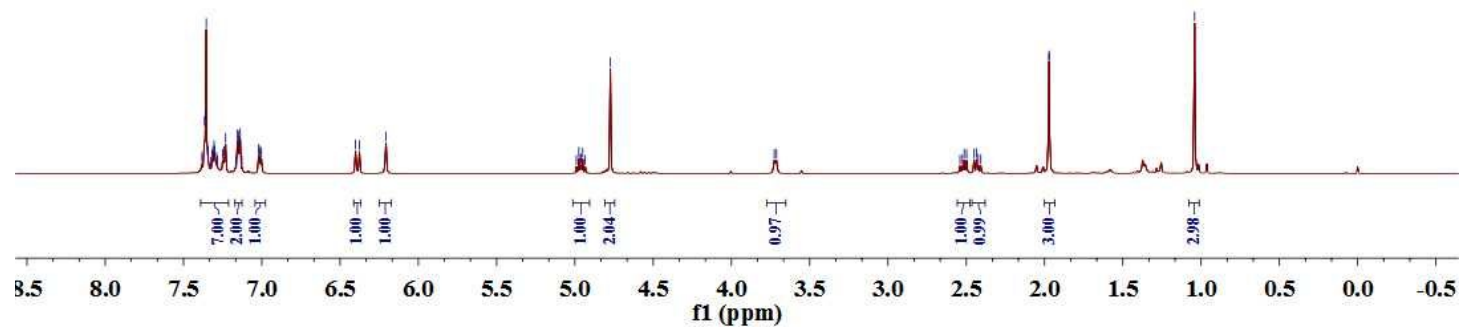

${ }^{1} \mathrm{H}$ NMR Spectra of $\mathbf{3 a b}\left(500 \mathrm{MHz}, \mathrm{CDCl}_{3}\right)$ 


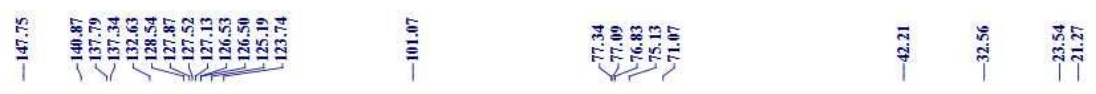

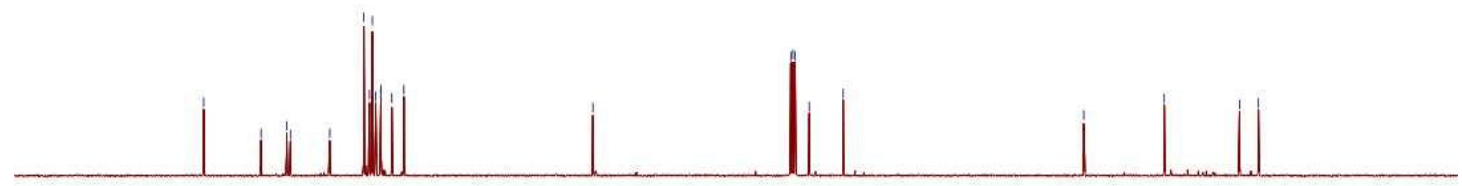

$\begin{array}{llllllllllllllllll}70 & 160 & 150 & 140 & 130 & 120 & 110 & 100 & \begin{array}{r}90 \\ \mathrm{f} 1(\mathrm{ppm})\end{array} & 70 & 60 & 50 & 40 & 30 & 20 & 10 & 0\end{array}$

${ }^{13} \mathrm{C}$ NMR Spectra of $3 a b\left(125 \mathrm{MHz}, \mathrm{CDCl}_{3}\right)$ 

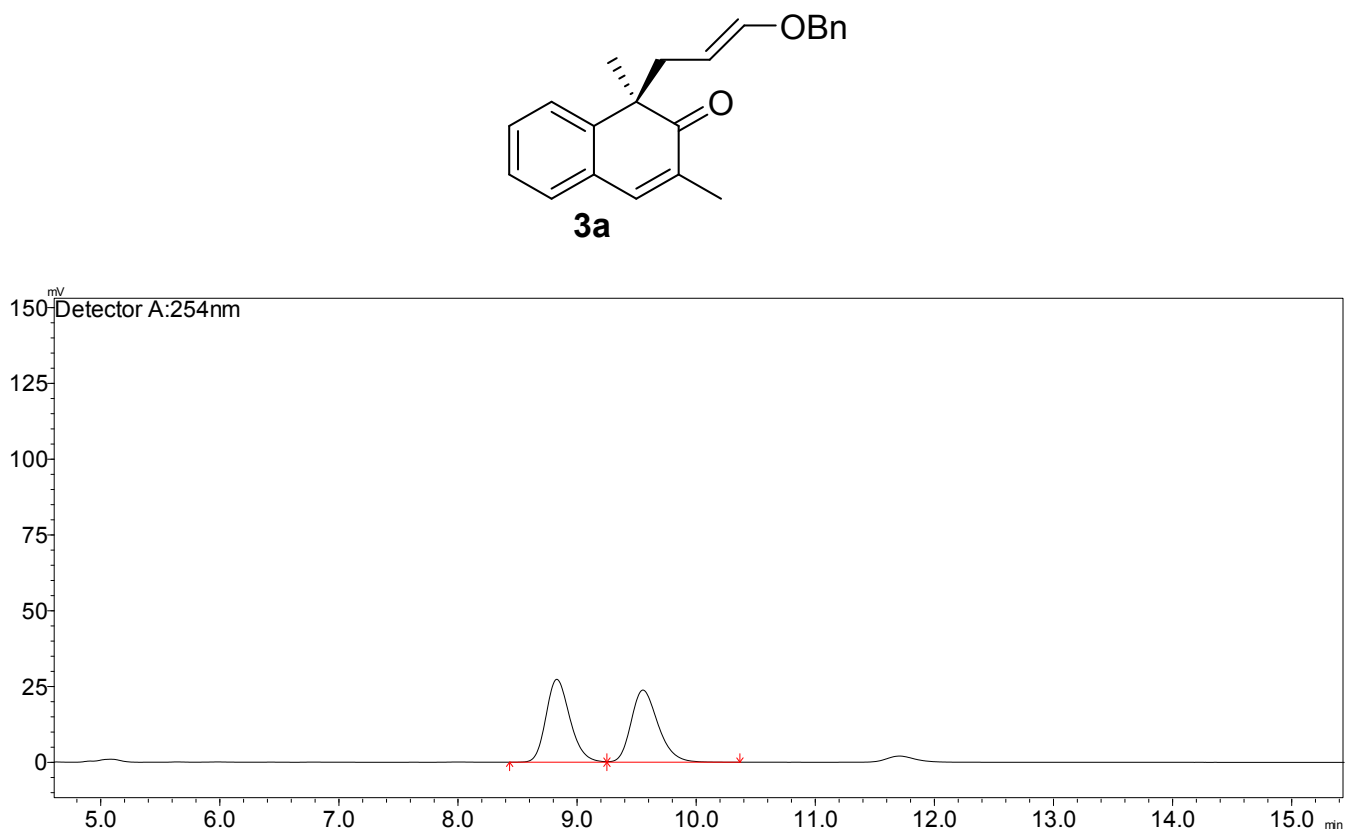

\begin{tabular}{|r|r|r|r|r|r|}
\hline Peak\# & Ret. Time & Area & Height & Area \% & Height \% \\
\hline 1 & 8.825 & 382591 & 27336 & 50.456 & 53.465 \\
\hline 2 & 9.549 & 375673 & 23793 & 49.544 & 46.535 \\
\hline Total & & 758264 & 51129 & 100.000 & 100.000 \\
\hline
\end{tabular}

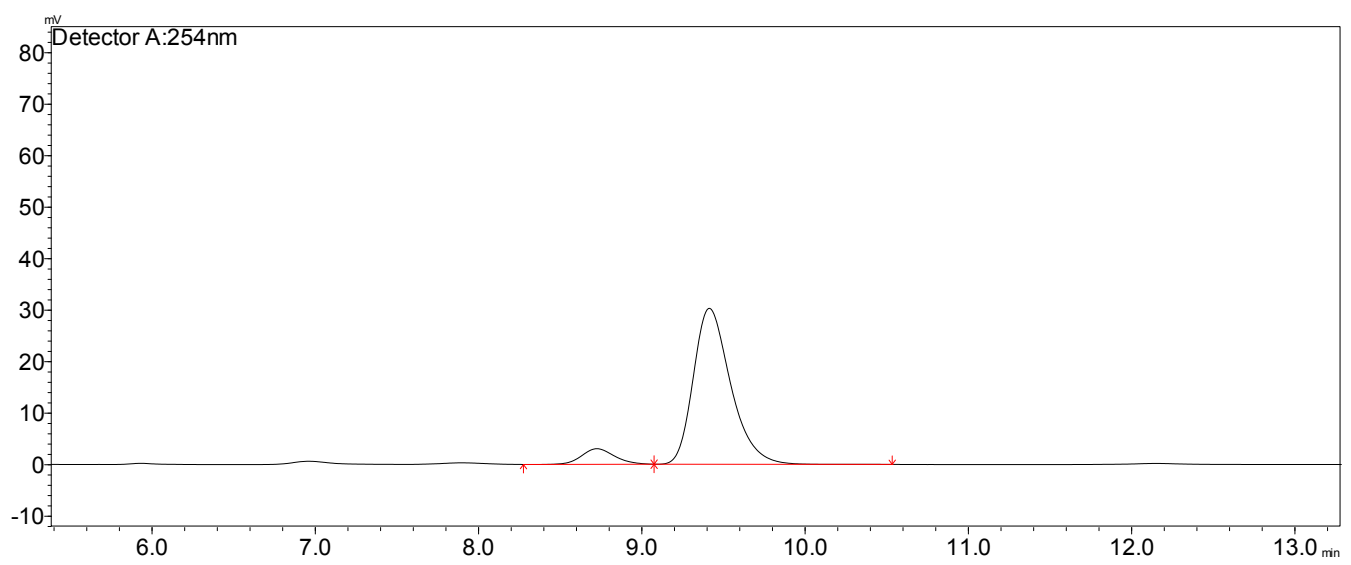

\begin{tabular}{|c|c|c|c|c|c|}
\hline Peak\# & Ret. Time & Area & Height & Area \% & Height \% \\
\hline 1 & 8.72 & 42403 & 3036 & 8.1 & 9.113 \\
\hline 2 & 9.409 & 481075 & 30278 & 91.9 & 90.887 \\
\hline Total & & 523478 & 33314 & 100 & 100 \\
\hline
\end{tabular}



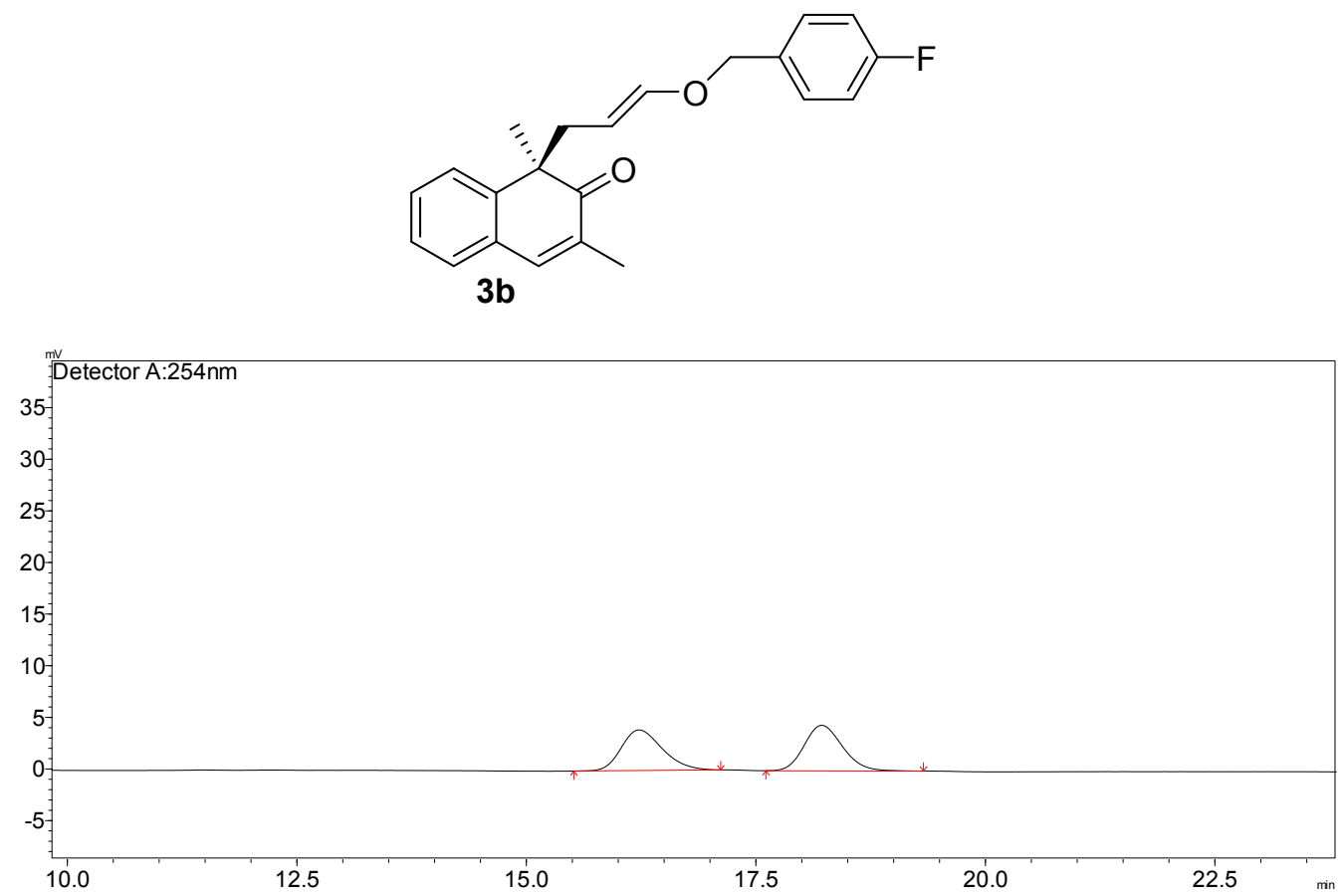

\begin{tabular}{|c|c|c|c|c|c|}
\hline Peak\# & Ret. Time & Area & Height & Area \% & Height \% \\
\hline 1 & 16.224 & 122224 & 3933 & 48.835 & 47.139 \\
\hline 2 & 18.213 & 128054 & 4411 & 51.165 & 52.861 \\
\hline Total & & 250277 & 8344 & 100 & 100 \\
\hline
\end{tabular}

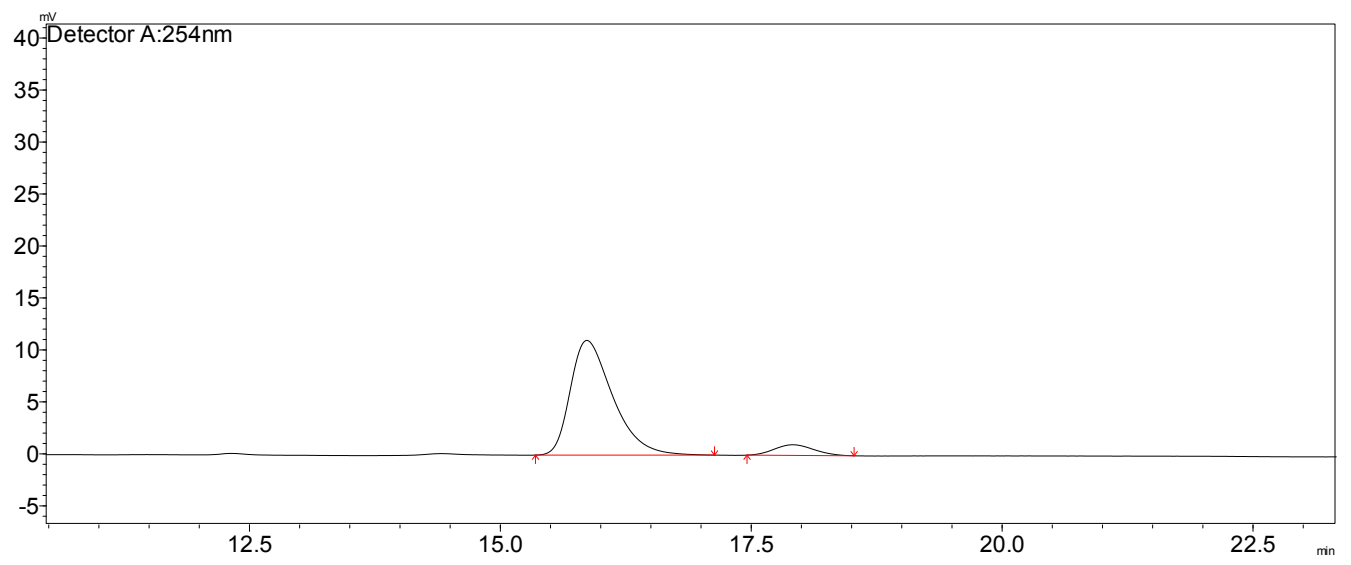

\begin{tabular}{|c|c|c|c|c|c|}
\hline Peak\# & Ret. Time & Area & Height & Area \% & Height \% \\
\hline 1 & 15.858 & 324569 & 11030 & 91.985 & 91.498 \\
\hline 2 & 17.91 & 28282 & 1025 & 8.015 & 8.502 \\
\hline Total & & 352851 & 12055 & 100 & 100 \\
\hline
\end{tabular}



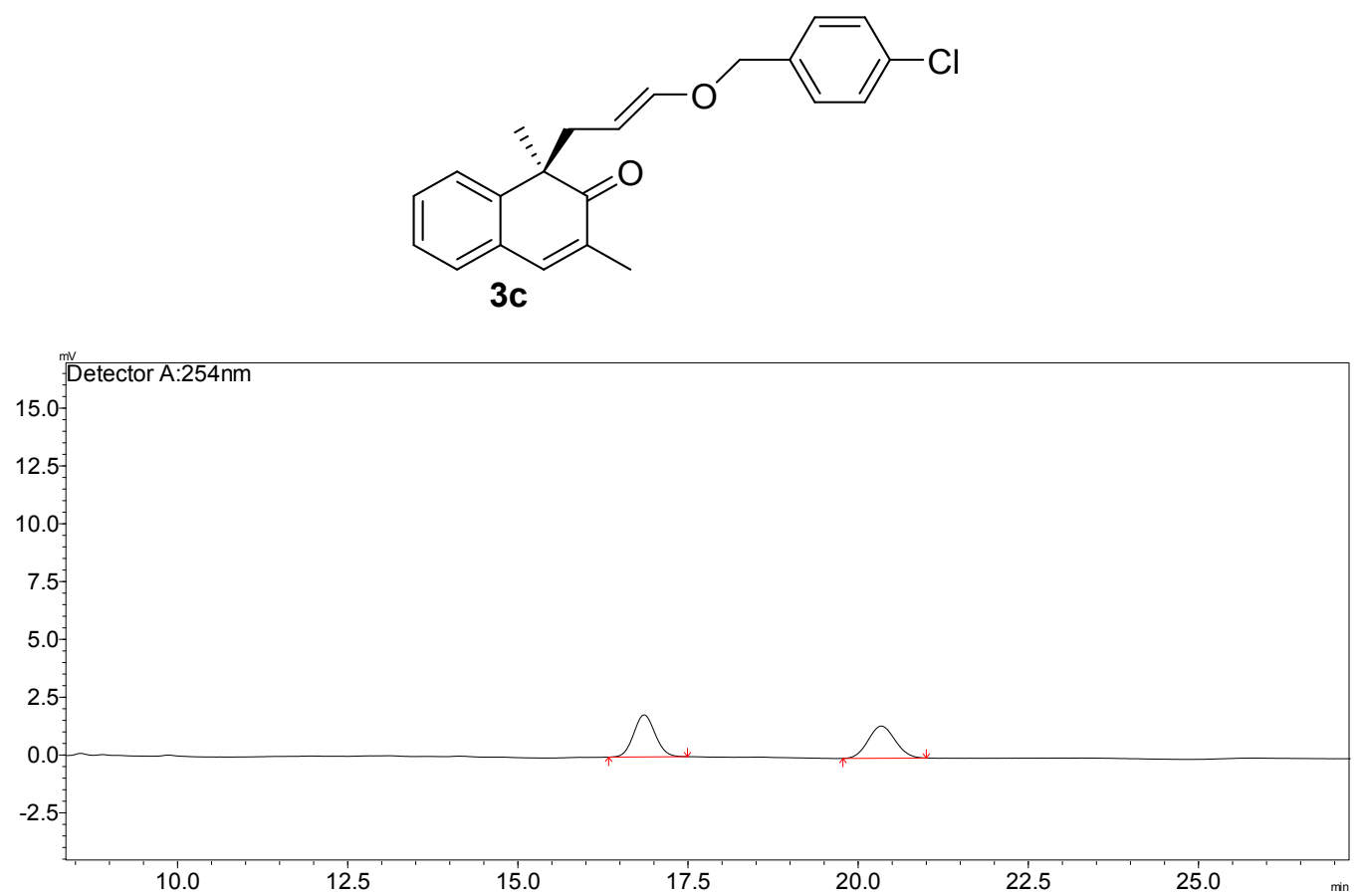

\begin{tabular}{|c|c|c|c|c|c|}
\hline Peak\# & Ret. Time & Area & Height & Area \% & Height \% \\
\hline 1 & 16.85 & 39975 & 1817 & 50.869 & 56.626 \\
\hline 2 & 20.333 & 38609 & 1392 & 49.131 & 43.374 \\
\hline Total & & 78584 & 3209 & 100 & 100 \\
\hline
\end{tabular}

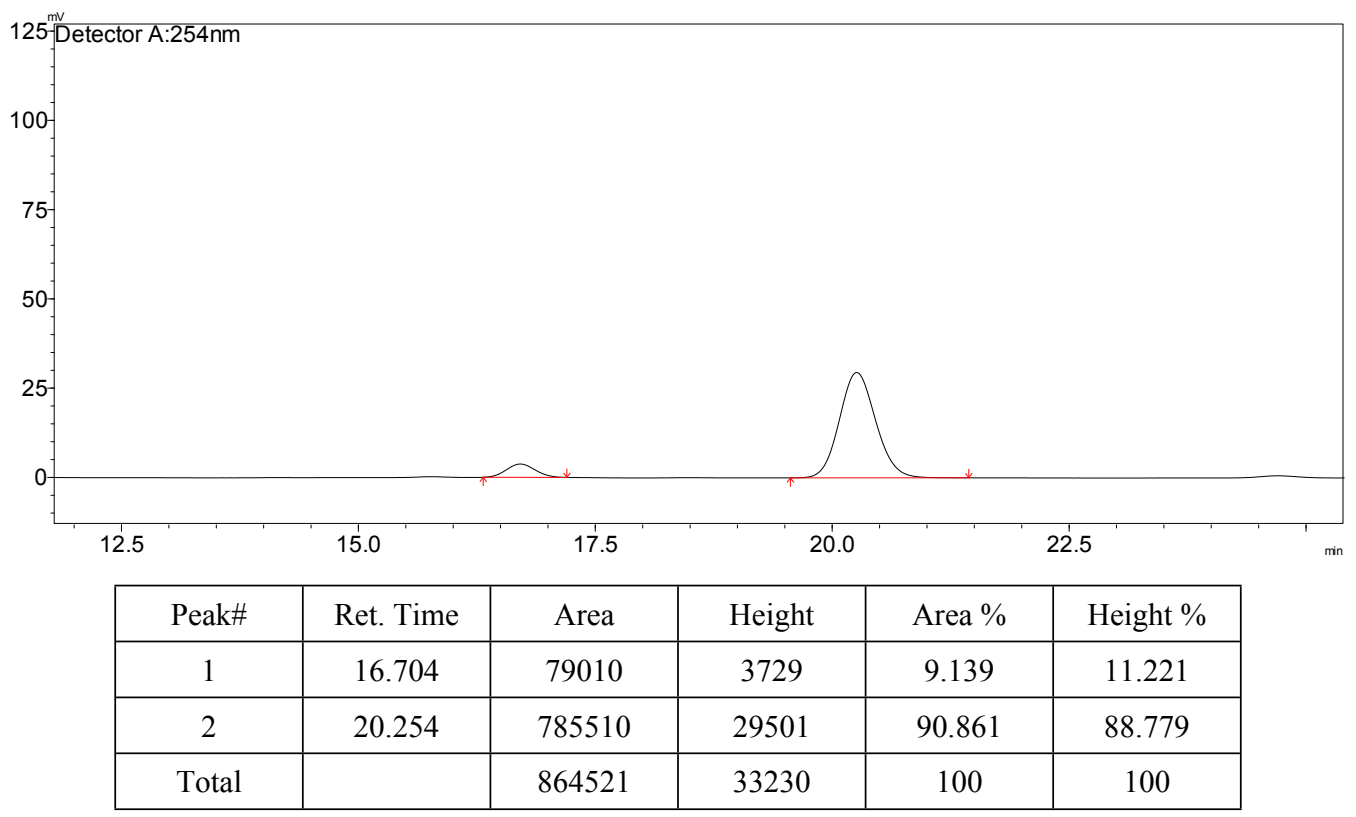



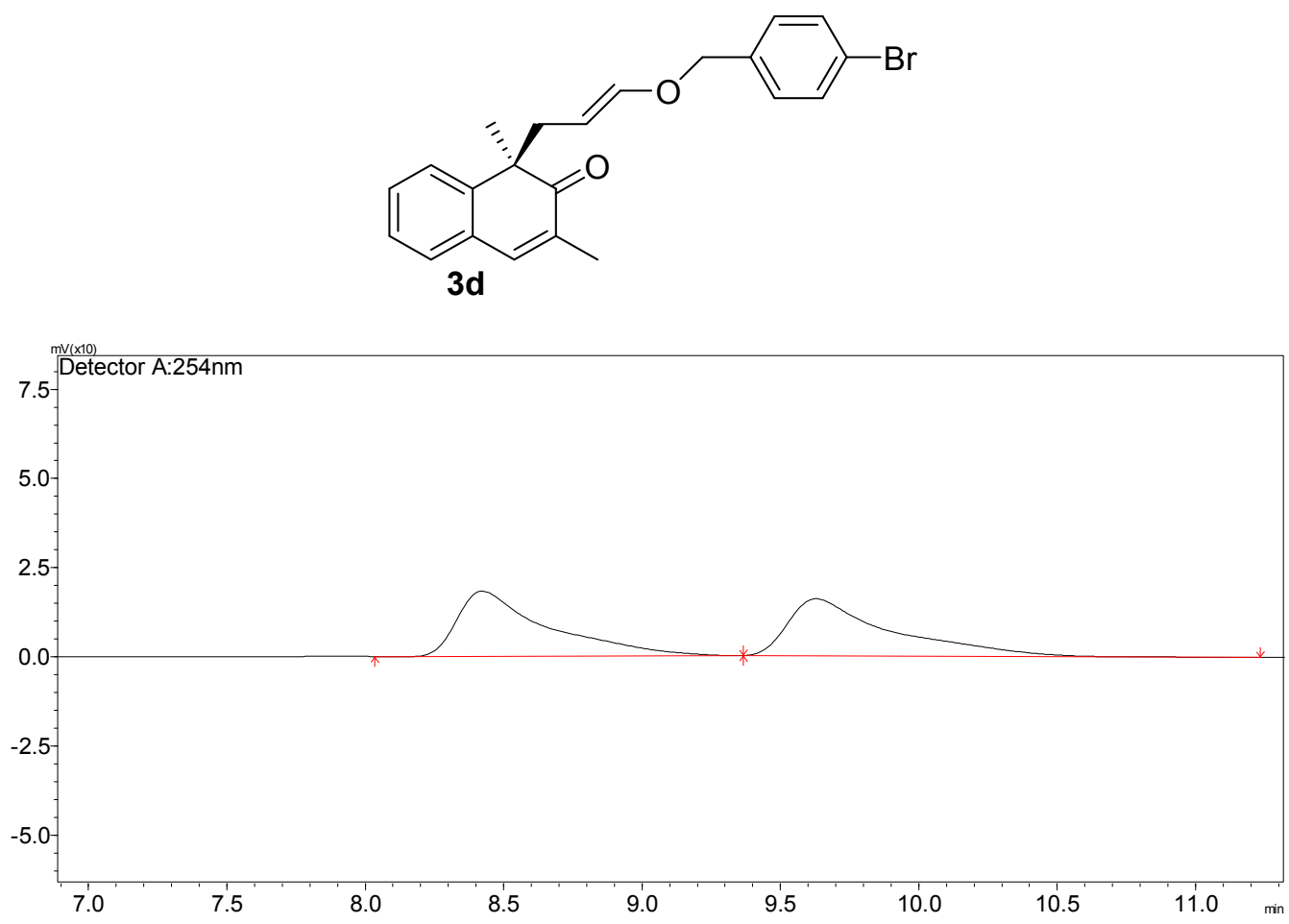

\begin{tabular}{|c|c|c|c|c|c|}
\hline Peak\# & Ret. Time & Area & Height & Area \% & Height \% \\
\hline 1 & 8.42 & 410028 & 18244 & 50.206 & 53.364 \\
\hline 2 & 9.625 & 406658 & 15944 & 49.794 & 46.636 \\
\hline Total & & 816686 & 34188 & 100 & 100 \\
\hline
\end{tabular}

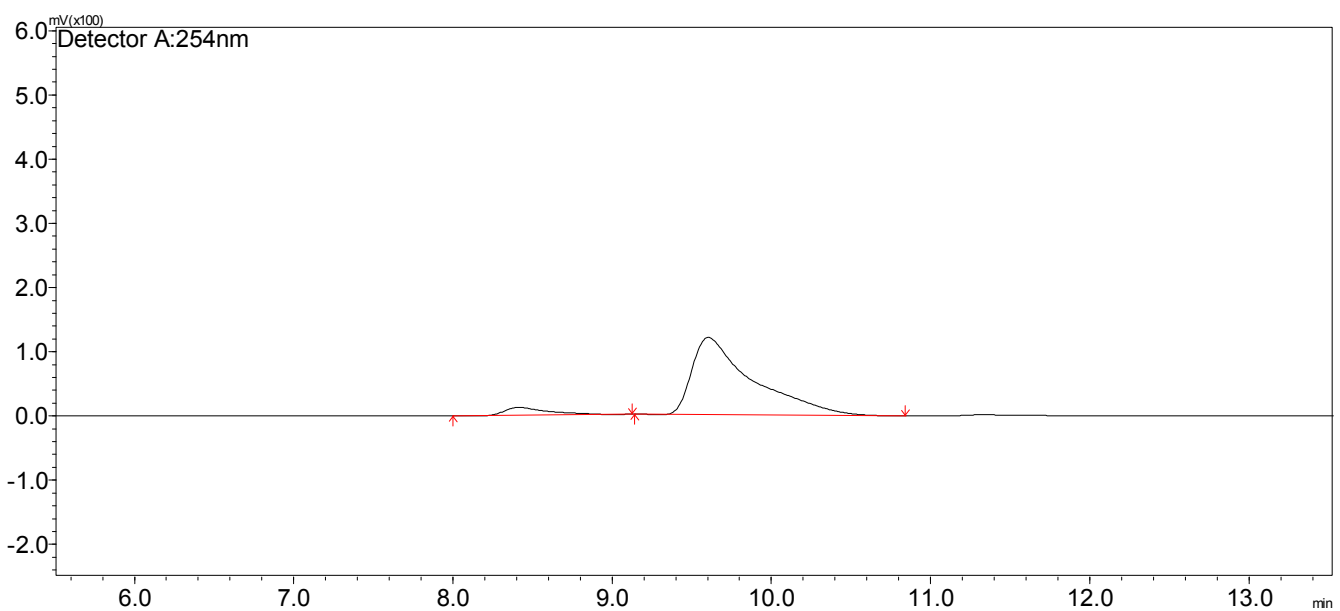

\begin{tabular}{|c|c|c|c|c|c|}
\hline Peak\# & Ret. Time & Area & Height & Area \% & Height \% \\
\hline 1 & 8.408 & 215822 & 12193 & 6.38 & 9.226 \\
\hline 2 & 9.6 & 3167032 & 119965 & 93.62 & 90.774 \\
\hline Total & & 3382854 & 132157 & 100 & 100 \\
\hline
\end{tabular}



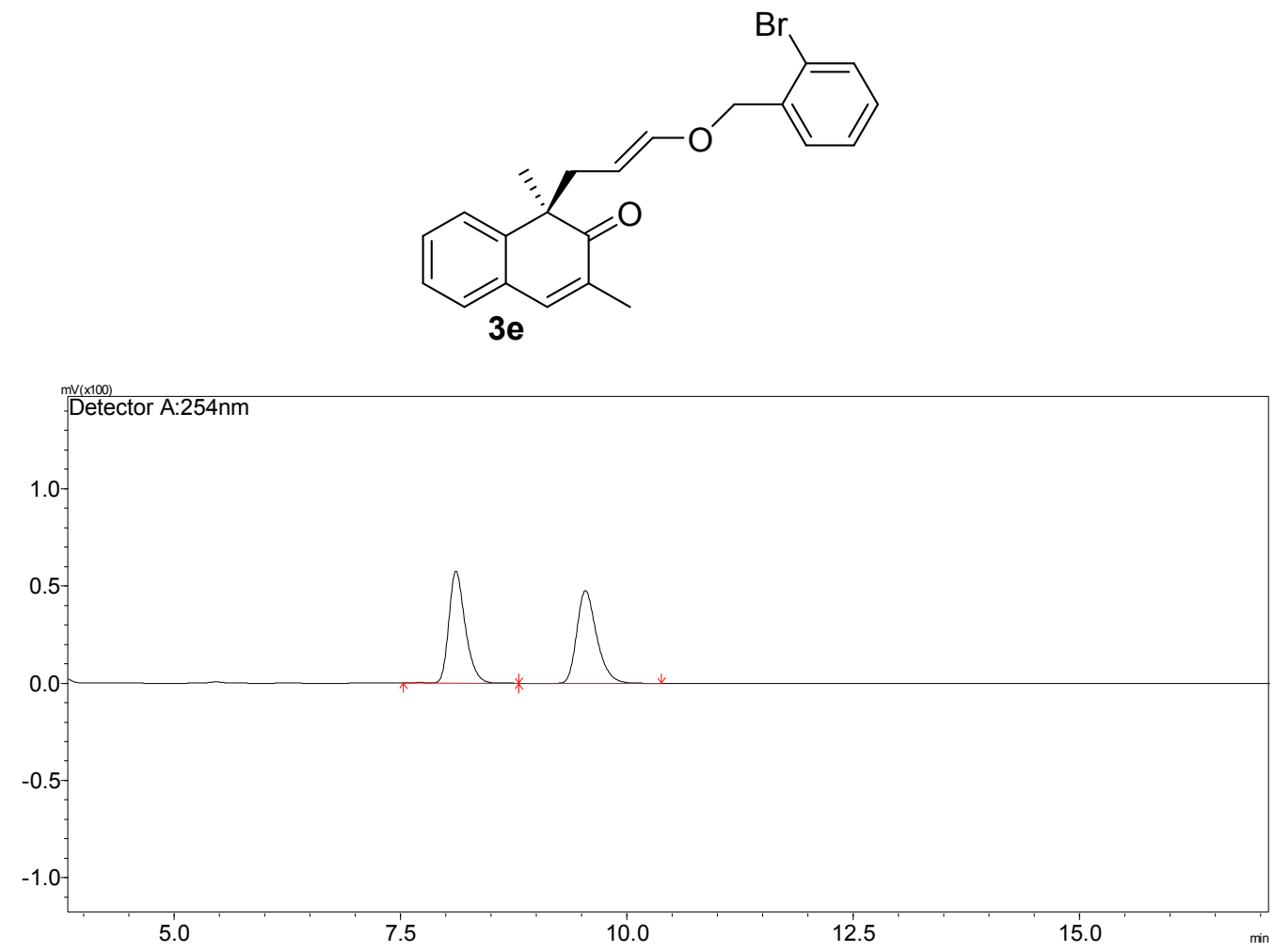

\begin{tabular}{|c|c|c|c|c|c|}
\hline Peak\# & Ret. Time & Area & Height & Area \% & Height \% \\
\hline 1 & 8.108 & 729839 & 57593 & 50.075 & 54.721 \\
\hline 2 & 9.539 & 727644 & 47656 & 49.925 & 45.279 \\
\hline Total & & 1457482 & 105249 & 100 & 100 \\
\hline
\end{tabular}

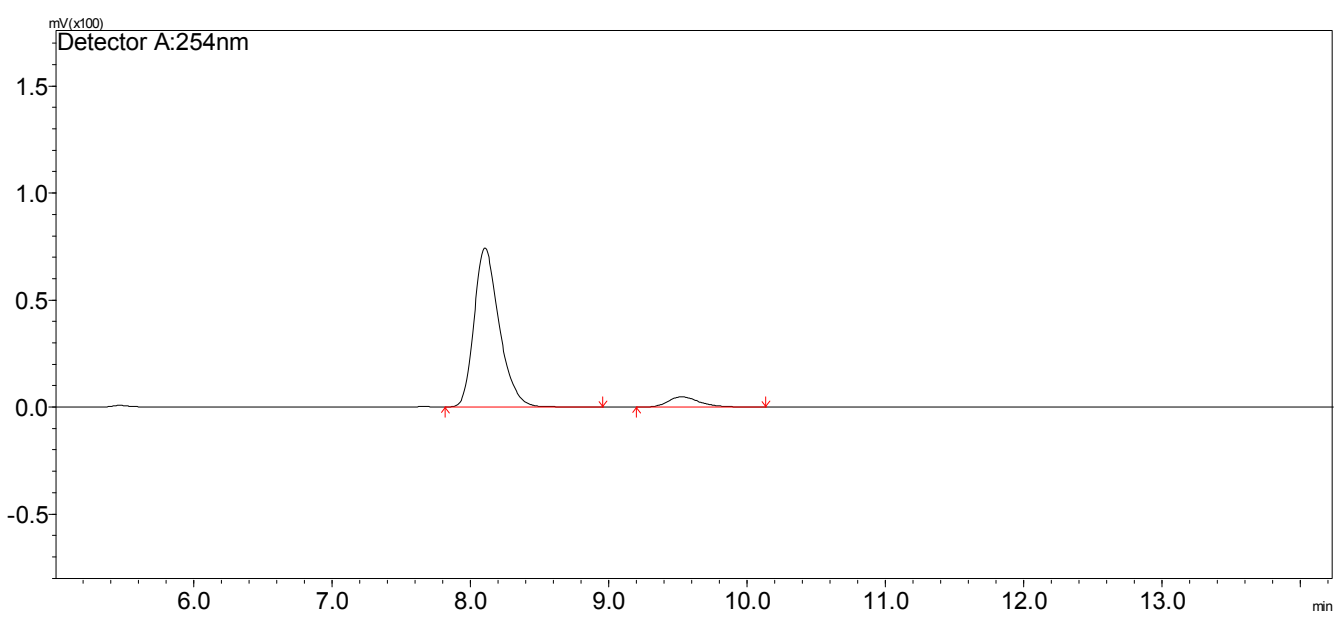

\begin{tabular}{|c|c|c|c|c|c|}
\hline Peak\# & Ret. Time & Area & Height & Area \% & Height \% \\
\hline 1 & 8.101 & 936785 & 74220 & 92.548 & 93.876 \\
\hline 2 & 9.524 & 75433 & 4842 & 7.452 & 6.124 \\
\hline Total & & 1012218 & 79062 & 100 & 100 \\
\hline
\end{tabular}



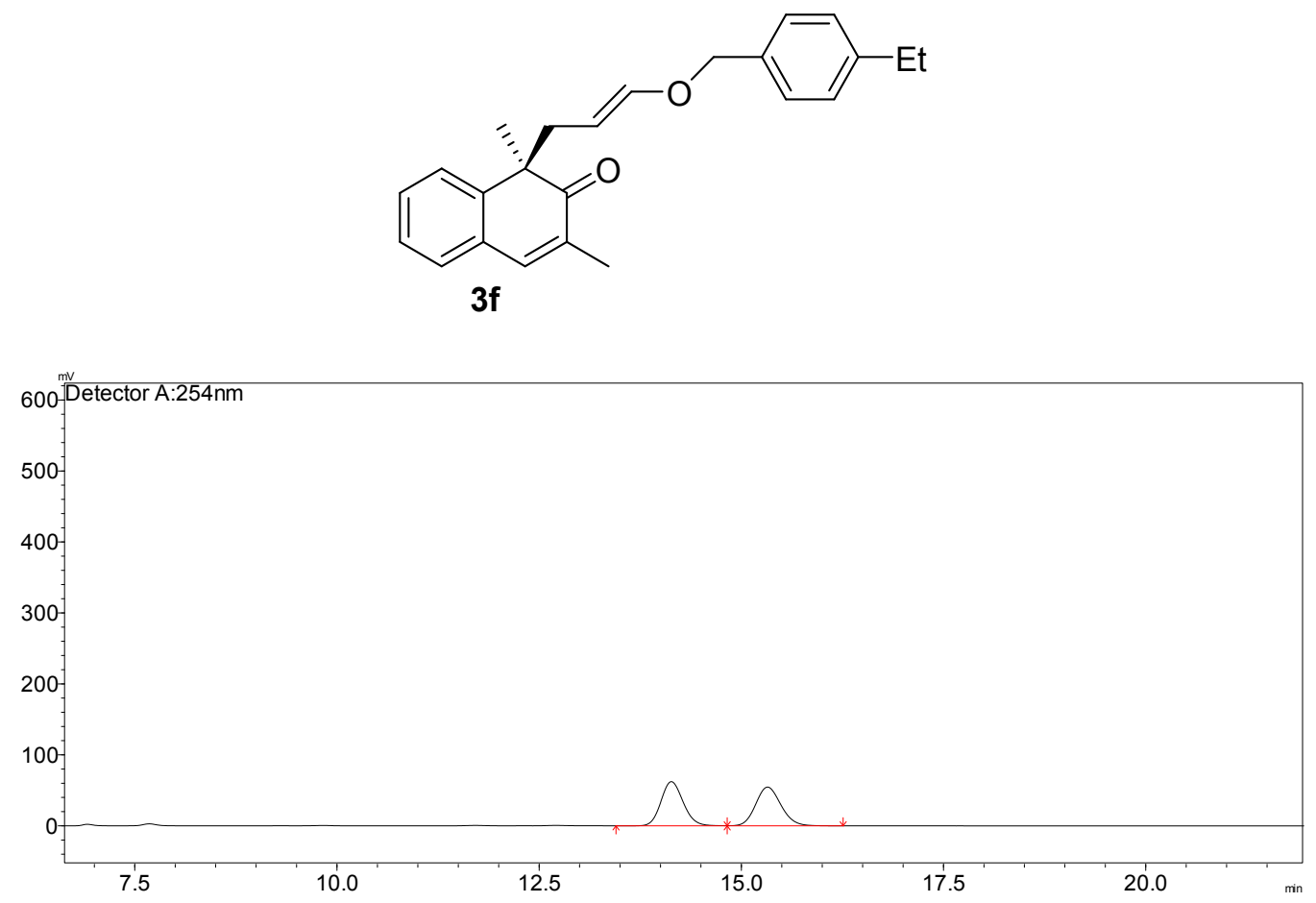

\begin{tabular}{|c|c|c|c|c|c|}
\hline Peak\# & Ret. Time & Area & Height & Area \% & Height \% \\
\hline 1 & 14.13 & 1191550 & 62296 & 50.052 & 53.269 \\
\hline 2 & 15.319 & 1189088 & 54650 & 49.948 & 46.731 \\
\hline Total & & 2380639 & 116946 & 100 & 100 \\
\hline
\end{tabular}

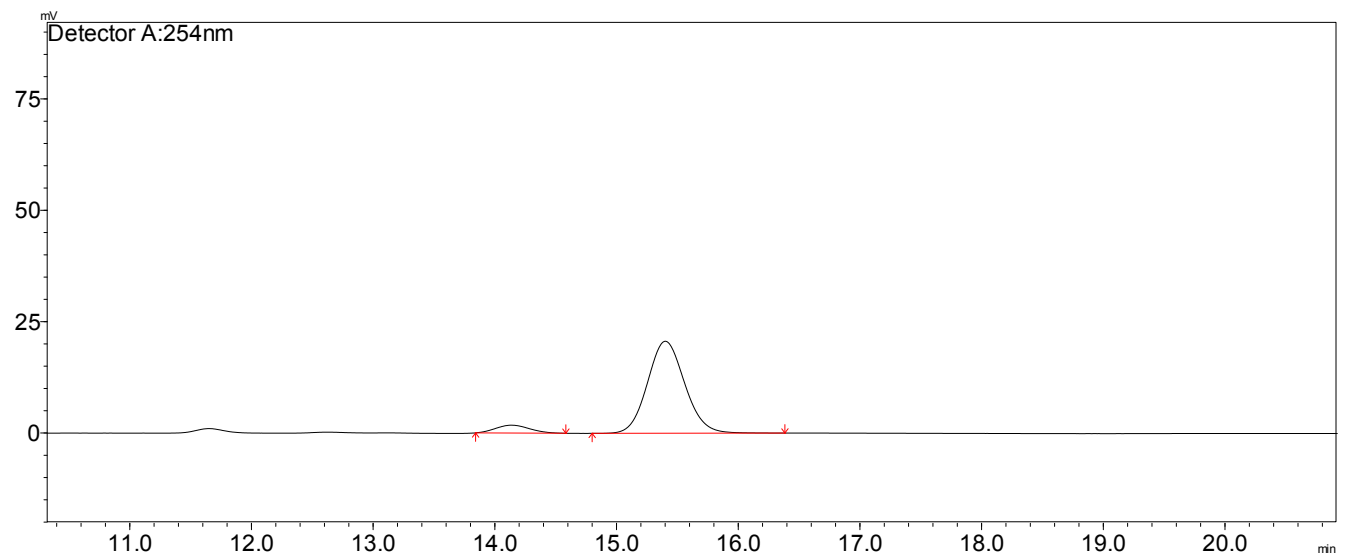

\begin{tabular}{|c|c|c|c|c|c|}
\hline Peak\# & Ret. Time & Area & Height & Area \% & Height \% \\
\hline 1 & 14.133 & 34051 & 1757 & 7.191 & 7.839 \\
\hline 2 & 15.397 & 439473 & 20659 & 92.809 & 92.161 \\
\hline Total & & 473524 & 22416 & 100 & 100 \\
\hline
\end{tabular}



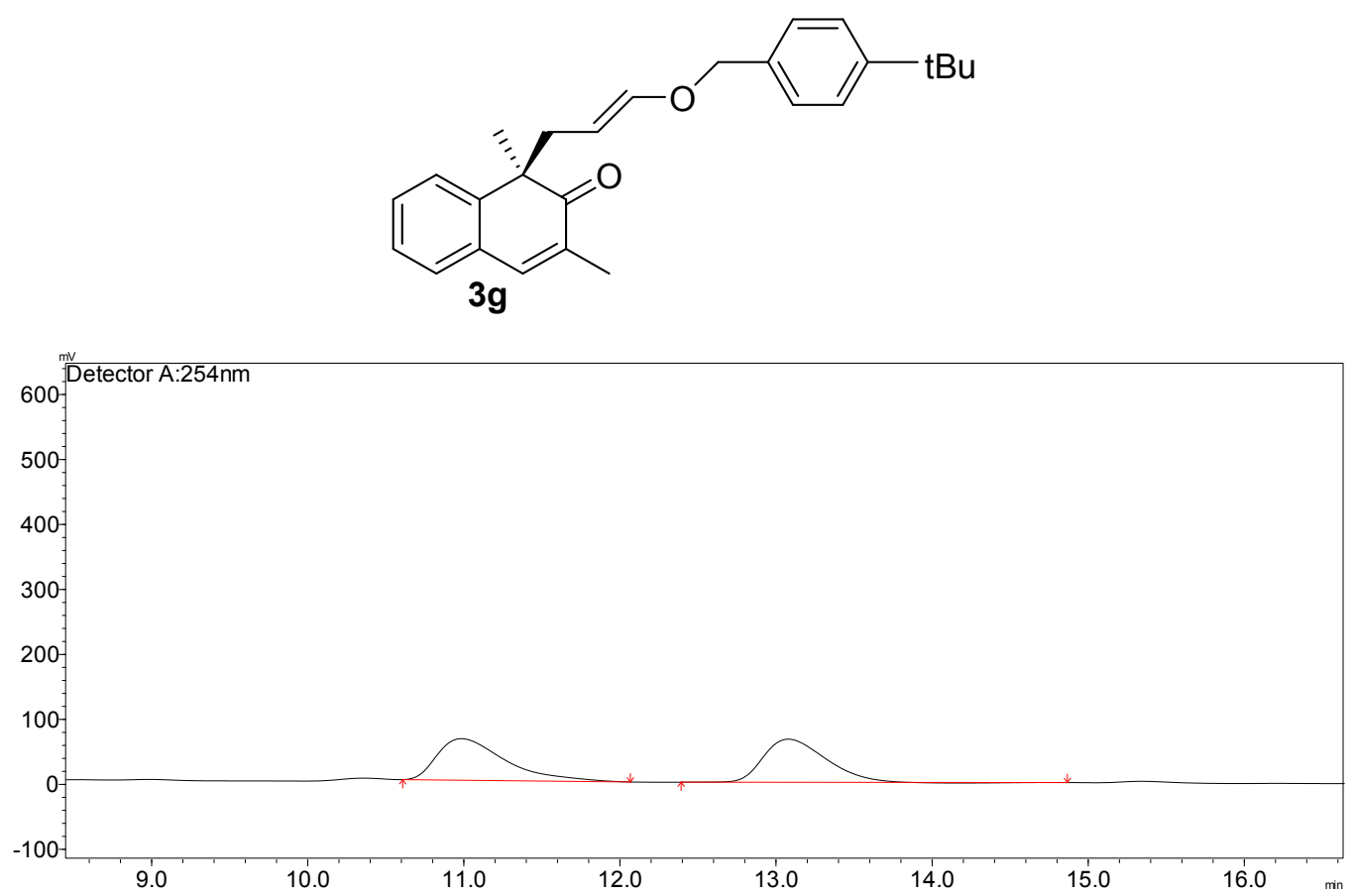

\begin{tabular}{|c|c|c|c|c|c|}
\hline Peak\# & Ret. Time & Area & Height & Area \% & Height \% \\
\hline 1 & 10.98 & 1939112 & 63928 & 51.471 & 49.08 \\
\hline 2 & 13.074 & 1828239 & 66325 & 48.529 & 50.92 \\
\hline Total & & 3767351 & 130253 & 100 & 100 \\
\hline
\end{tabular}

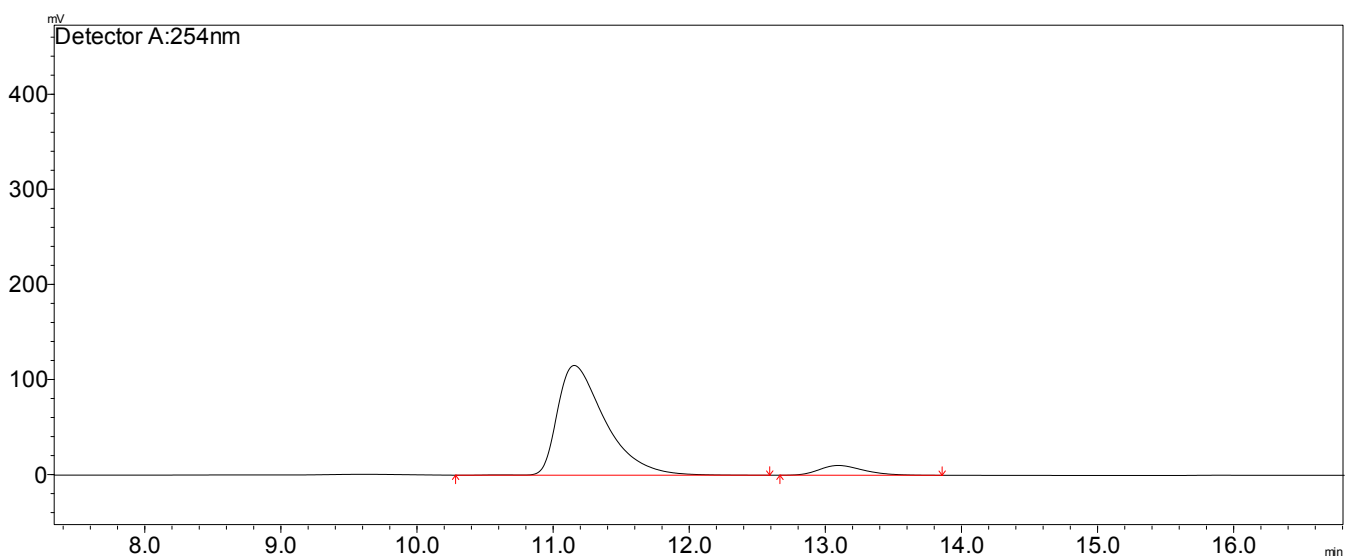

\begin{tabular}{|c|c|c|c|c|c|}
\hline Peak\# & Ret. Time & Area & Height & Area \% & Height $\%$ \\
\hline 1 & 11.151 & 2805685 & 115422 & 92.328 & 91.82 \\
\hline 2 & 13.091 & 233151 & 10283 & 7.672 & 8.18 \\
\hline Total & & 3038836 & 125705 & 100 & 100 \\
\hline
\end{tabular}



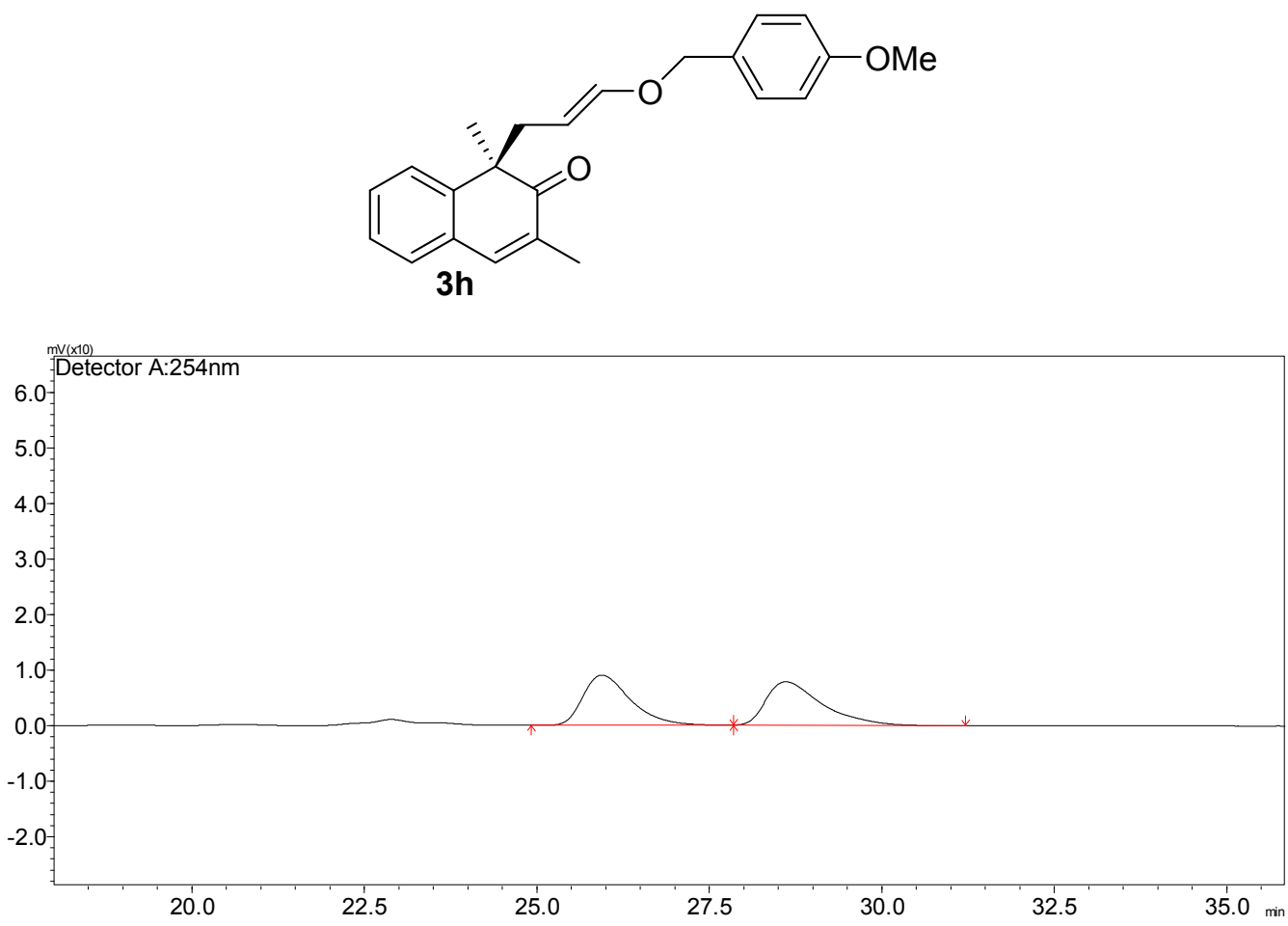

\begin{tabular}{|c|c|c|c|c|c|}
\hline Peak\# & Ret. Time & Area & Height & Area \% & Height \% \\
\hline 1 & 25.934 & 431442 & 9032 & 49.323 & 53.534 \\
\hline 2 & 28.605 & 443283 & 7839 & 50.677 & 46.466 \\
\hline Total & & 874725 & 16871 & 100 & 100 \\
\hline
\end{tabular}

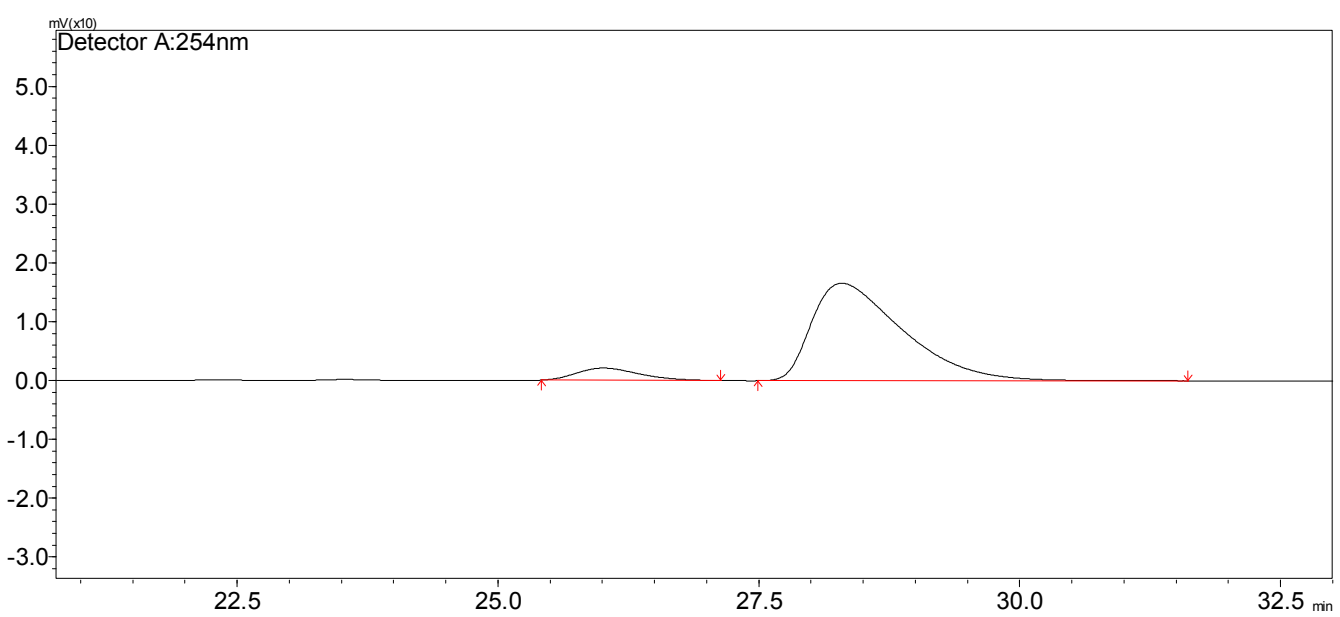

\begin{tabular}{|c|c|c|c|c|c|}
\hline Peak\# & Ret. Time & Area & Height & Area \% & Height \% \\
\hline 1 & 26.003 & 86184 & 2076 & 7.899 & 11.128 \\
\hline 2 & 28.29 & 1004889 & 16581 & 92.101 & 88.872 \\
\hline Total & & 1091072 & 18657 & 100 & 100 \\
\hline
\end{tabular}



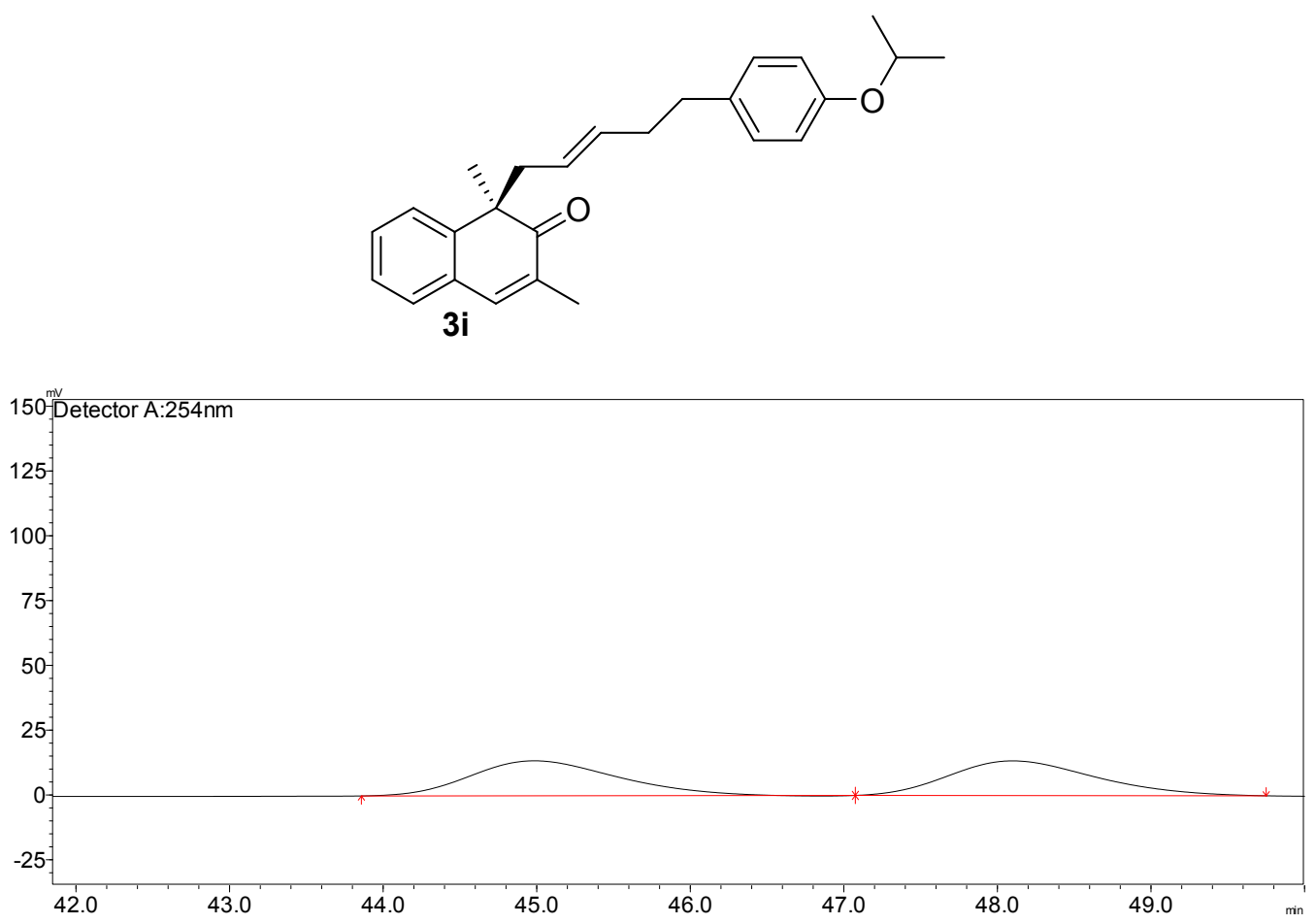

\begin{tabular}{|c|c|c|c|c|c|}
\hline Peak\# & Ret. Time & Area & Height & Area \% & Height \% \\
\hline 1 & 44.98 & 890548 & 13497 & 50.108 & 50.204 \\
\hline 2 & 48.096 & 886693 & 13388 & 49.892 & 49.796 \\
\hline Total & & 1777241 & 26885 & 100 & 100 \\
\hline
\end{tabular}

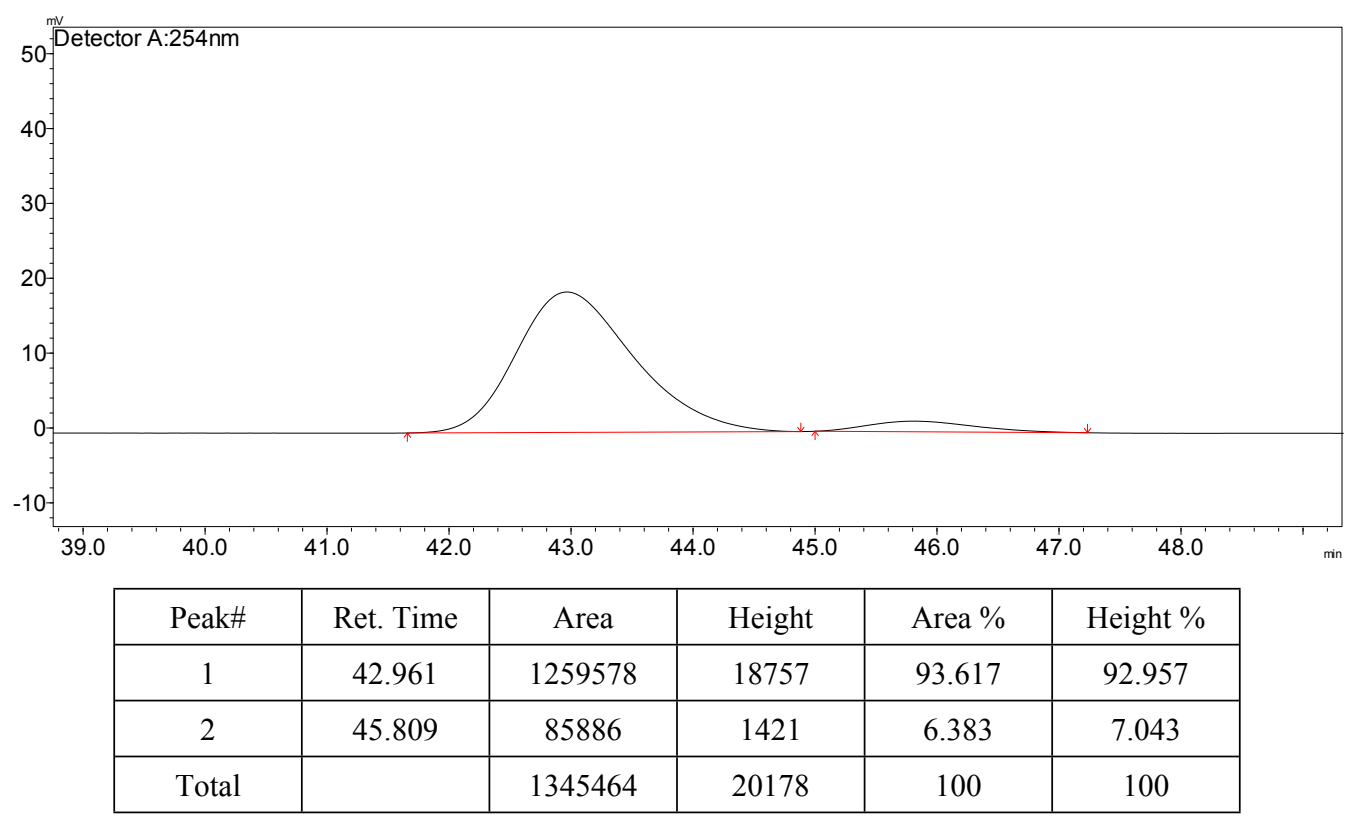



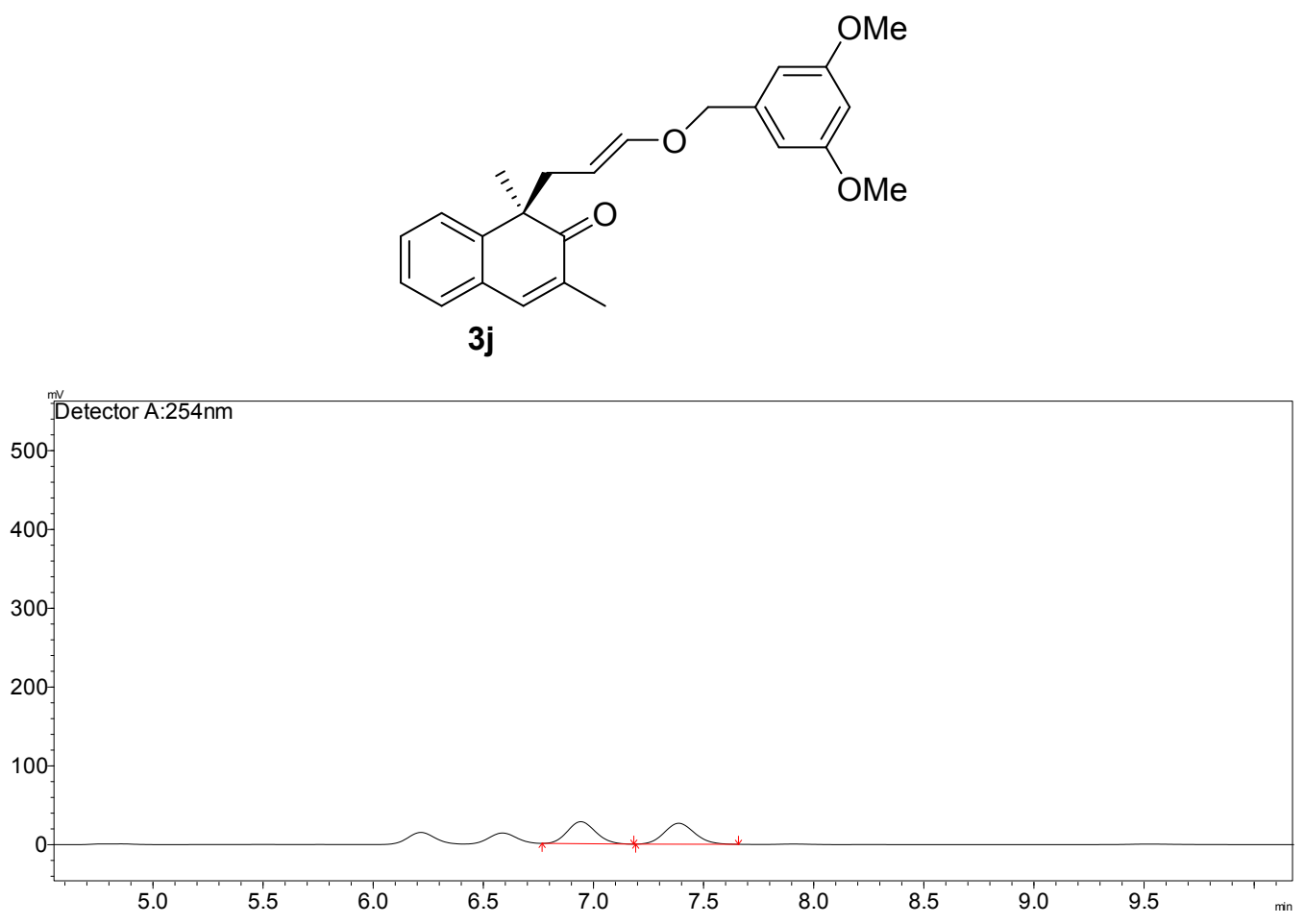

\begin{tabular}{|c|c|c|c|c|c|}
\hline Peak\# & Ret. Time & Area & Height & Area \% & Height \% \\
\hline 1 & 6.938 & 248557 & 27974 & 49.391 & 51.325 \\
\hline 2 & 7.383 & 254686 & 26529 & 50.609 & 48.675 \\
\hline Total & & 503243 & 54503 & 100 & 100 \\
\hline
\end{tabular}

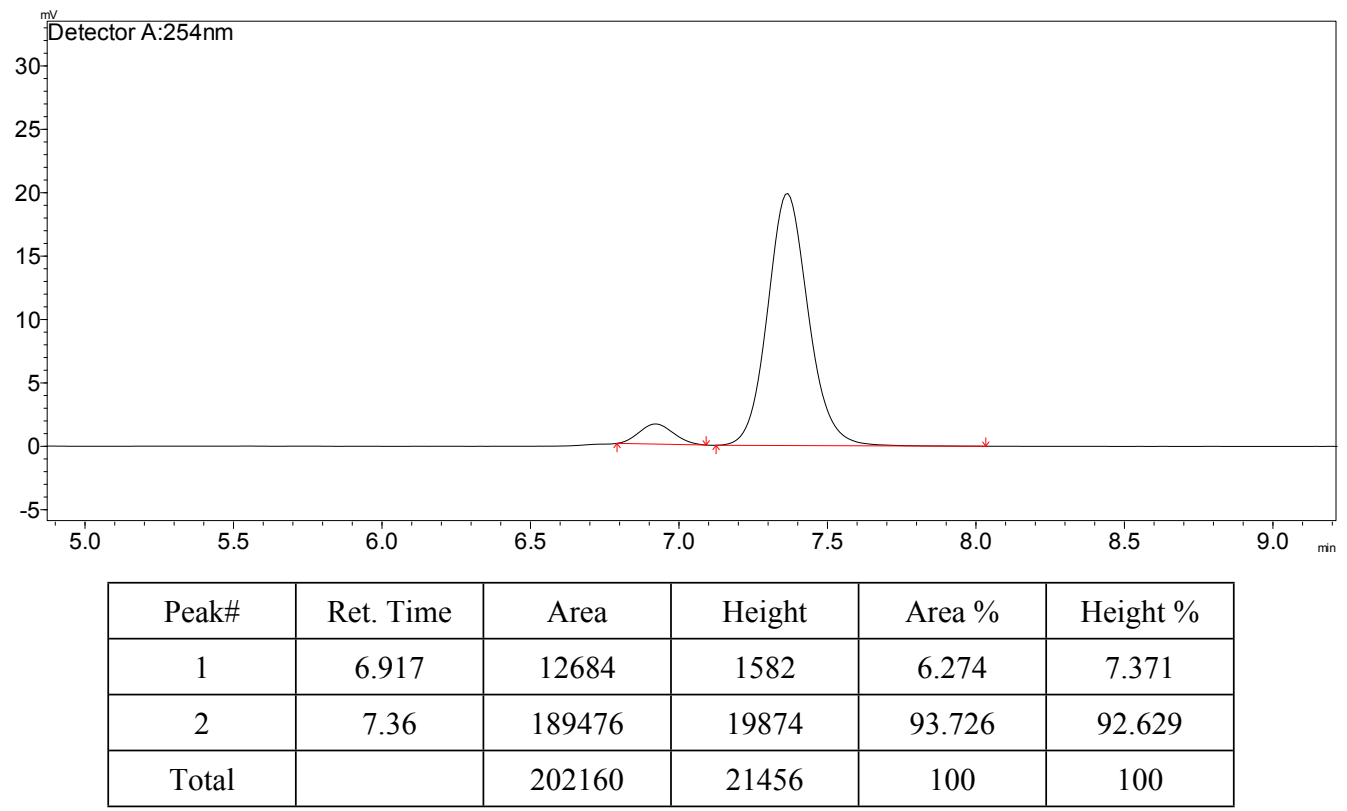



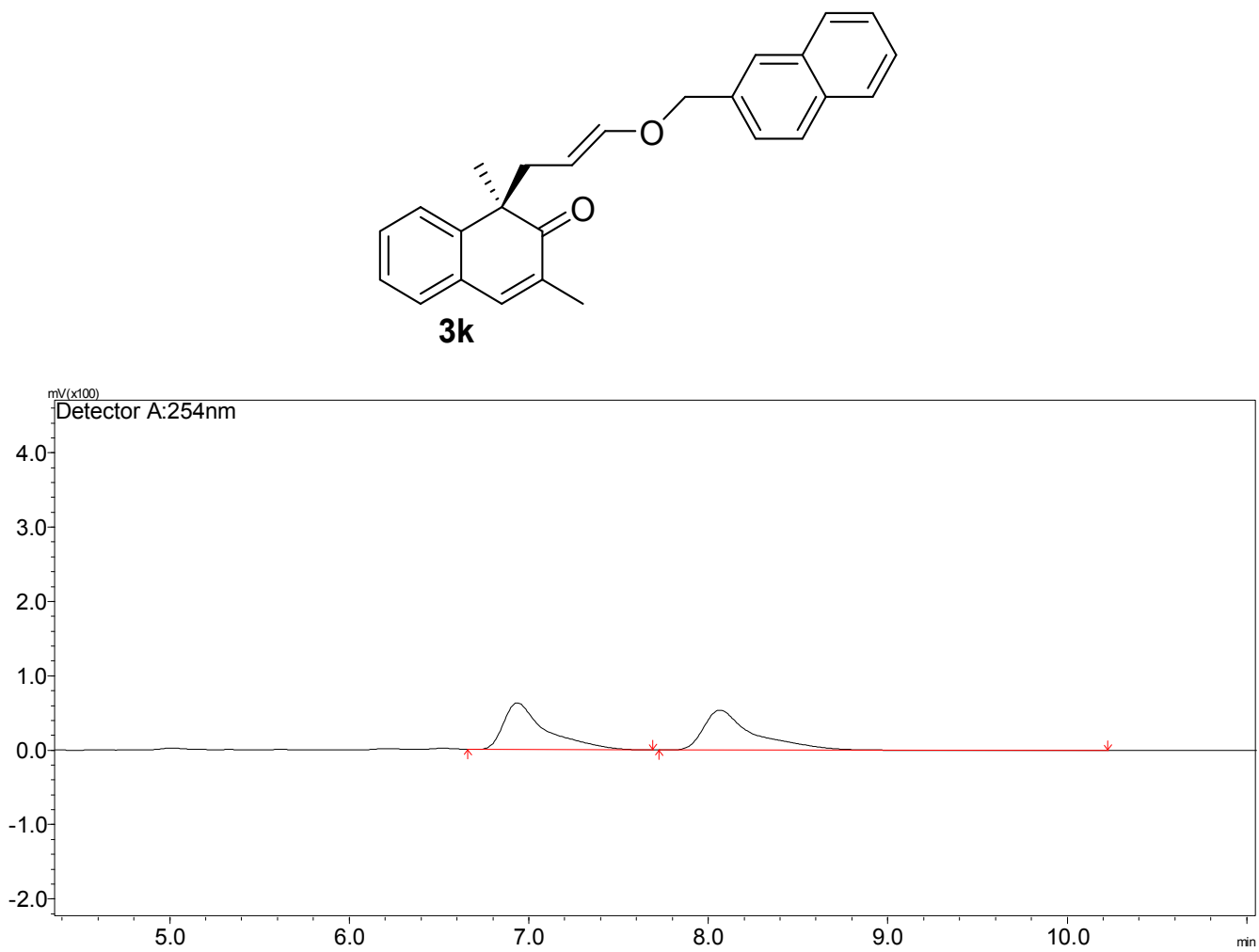

\begin{tabular}{|c|c|c|c|c|c|}
\hline Peak\# & Ret. Time & Area & Height & Area \% & Height \% \\
\hline 1 & 6.932 & 1031760 & 62893 & 49.793 & 53.928 \\
\hline 2 & 8.06 & 1040343 & 53730 & 50.207 & 46.072 \\
\hline Total & & 2072103 & 116622 & 100 & 100 \\
\hline
\end{tabular}

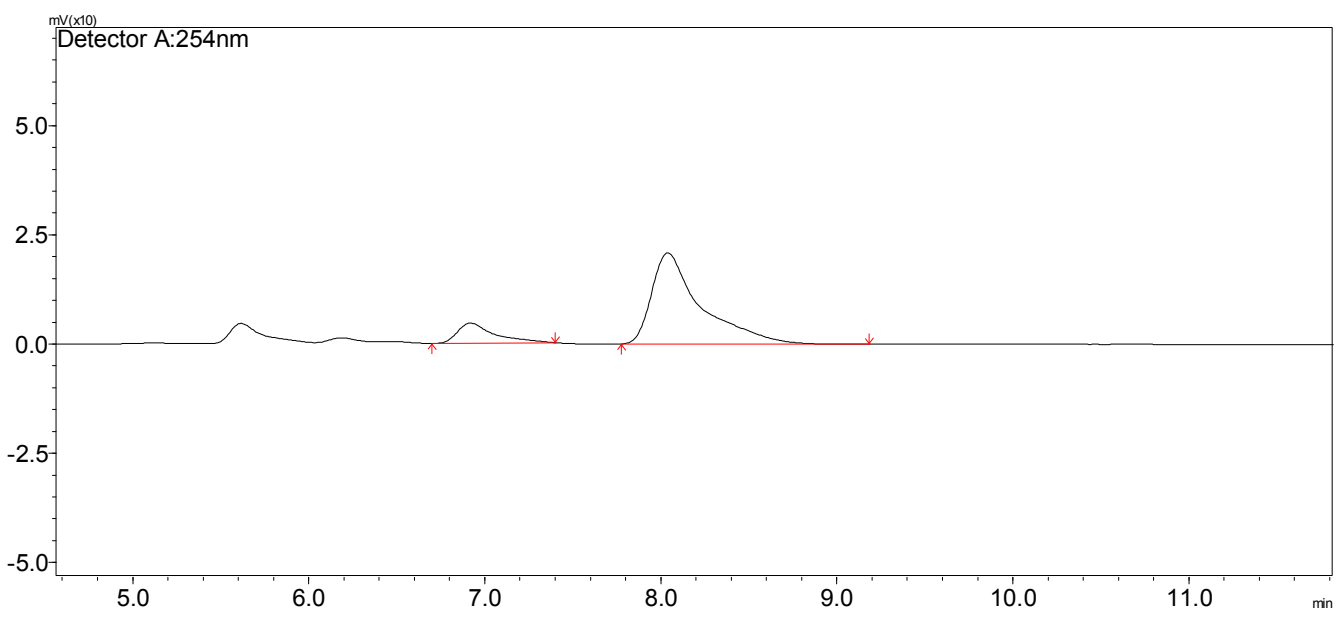

\begin{tabular}{|c|c|c|c|c|c|}
\hline Peak\# & Ret. Time & Area & Height & Area \% & Height \% \\
\hline 1 & 6.915 & 40993 & 3888 & 9.197 & 15.725 \\
\hline 2 & 8.035 & 404705 & 20838 & 90.803 & 84.275 \\
\hline Total & & 445698 & 24727 & 100 & 100 \\
\hline
\end{tabular}



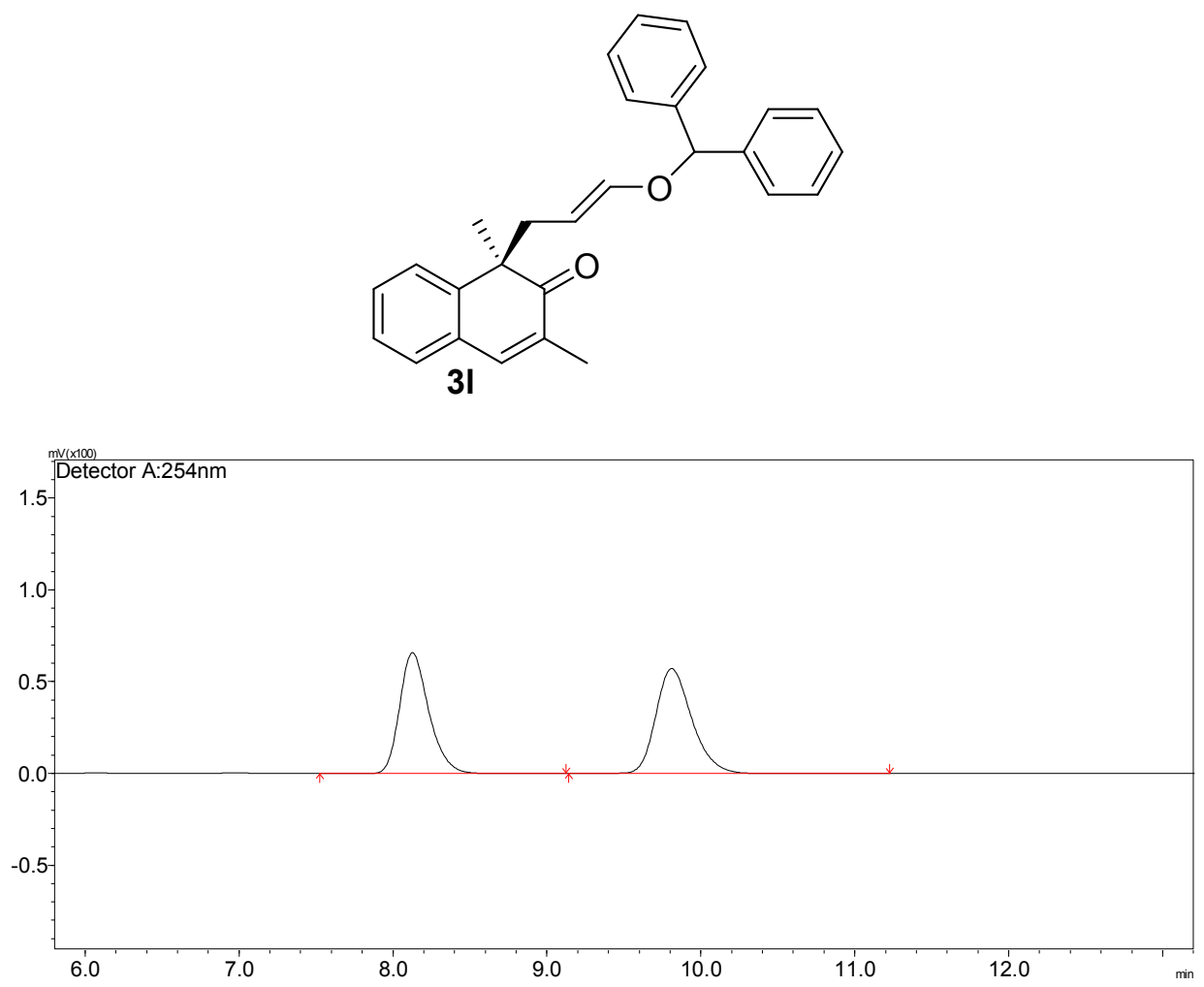

\begin{tabular}{|c|c|c|c|c|c|}
\hline Peak\# & Ret. Time & Area & Height & Area \% & Height \% \\
\hline 1 & 8.122 & 869487 & 65806 & 48.301 & 53.501 \\
\hline 2 & 9.806 & 930645 & 57192 & 51.699 & 46.499 \\
\hline Total & & 1800132 & 122998 & 100 & 100 \\
\hline
\end{tabular}

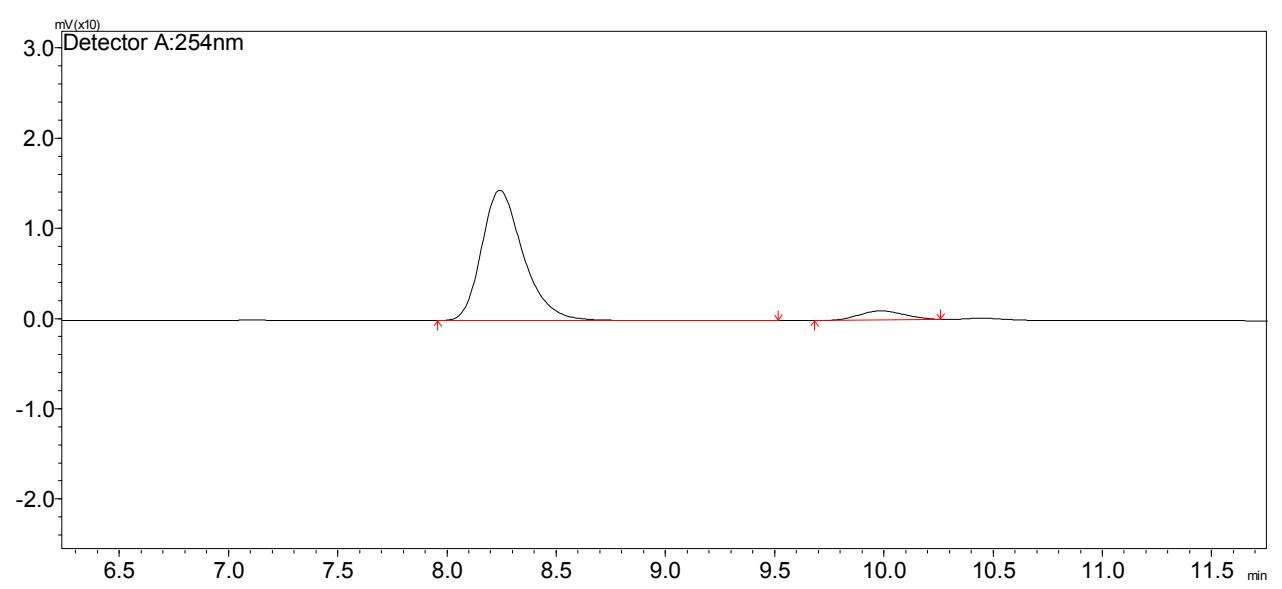

\begin{tabular}{|c|c|c|c|c|c|}
\hline Peak\# & Ret. Time & Area & Height & Area \% & Height \% \\
\hline 1 & 8.237 & 192673 & 14469 & 92.903 & 93.46 \\
\hline 2 & 9.982 & 14718 & 1012 & 7.097 & 6.54 \\
\hline Total & & 207391 & 15481 & 100 & 100 \\
\hline
\end{tabular}



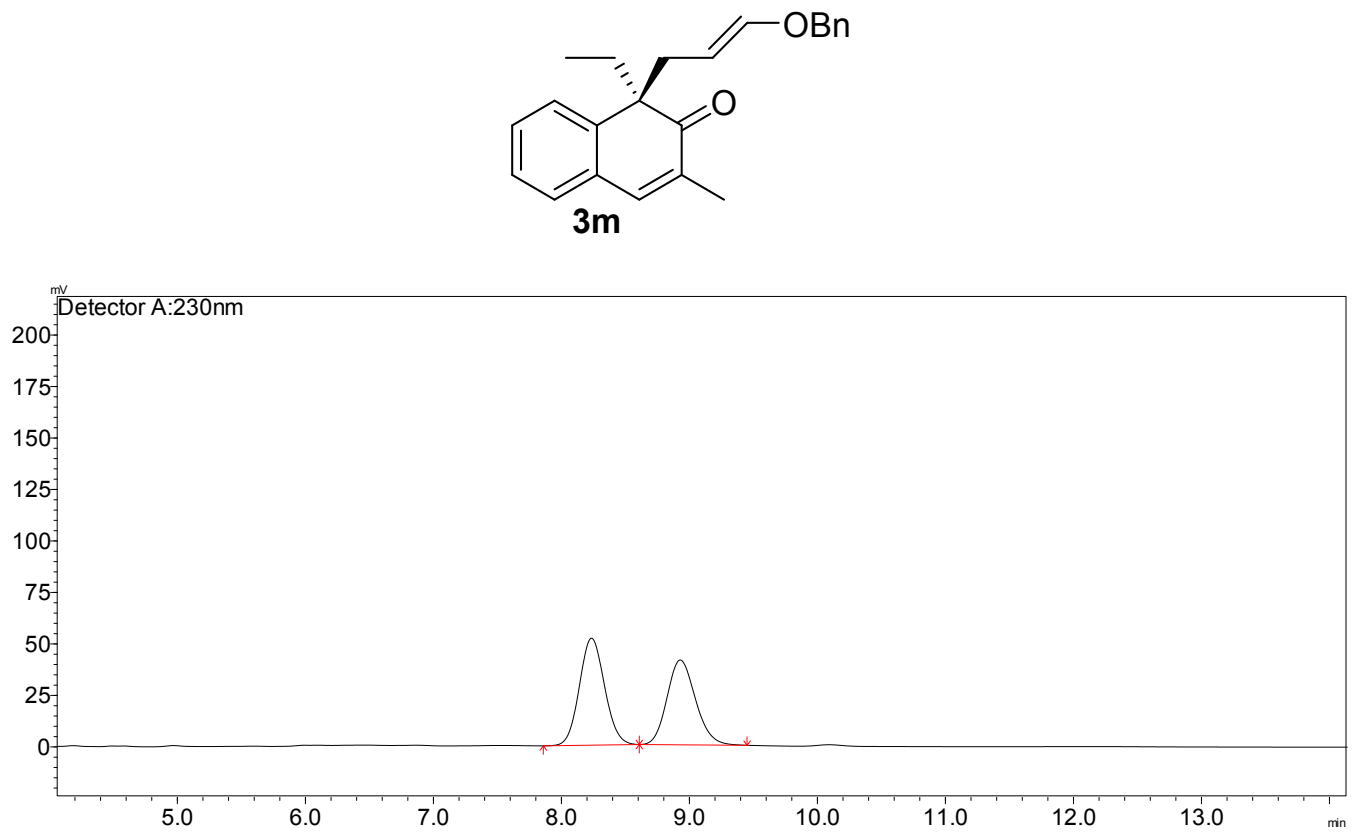

\begin{tabular}{|c|c|c|c|c|c|}
\hline Peak\# & Ret. Time & Area & Height & Area \% & Height \% \\
\hline 1 & 8.231 & 711582 & 51976 & 52.775 & 55.793 \\
\hline 2 & 8.924 & 636741 & 41183 & 47.225 & 44.207 \\
\hline Total & & 1348323 & 93159 & 100 & 100 \\
\hline
\end{tabular}

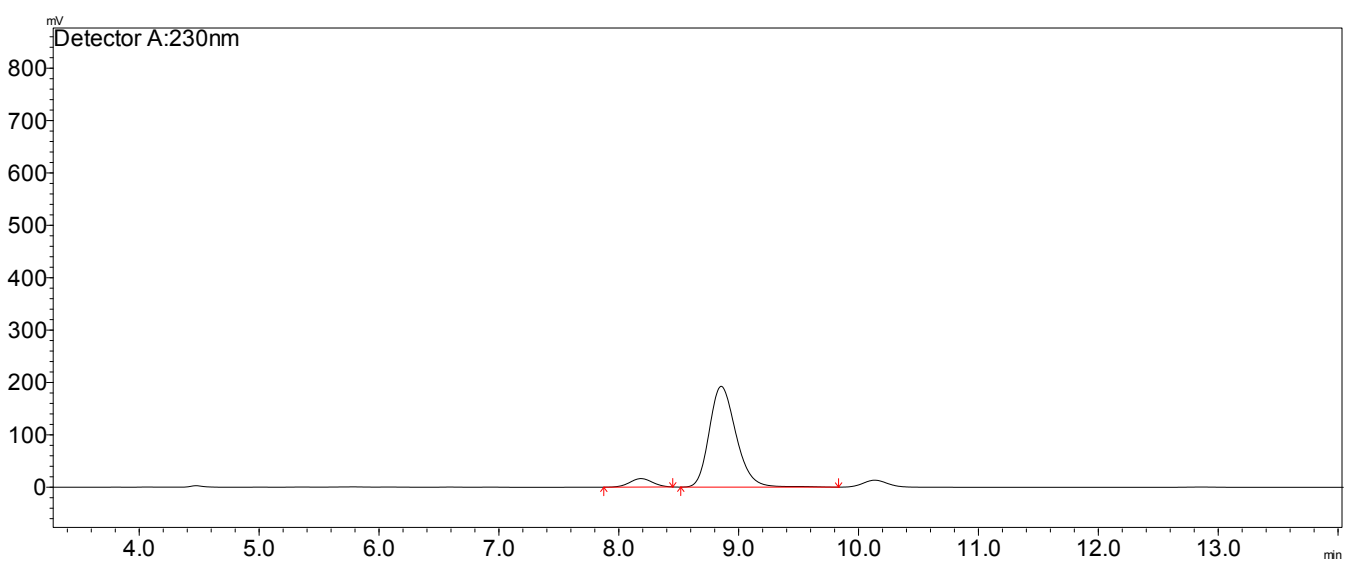

\begin{tabular}{|c|c|c|c|c|c|}
\hline Peak\# & Ret. Time & Area & Height & Area \% & Height \% \\
\hline 1 & 8.181 & 213235 & 16147 & 6.656 & 7.746 \\
\hline 2 & 8.85 & 2990506 & 192310 & 93.344 & 92.254 \\
\hline Total & & 3203742 & 208457 & 100 & 100 \\
\hline
\end{tabular}



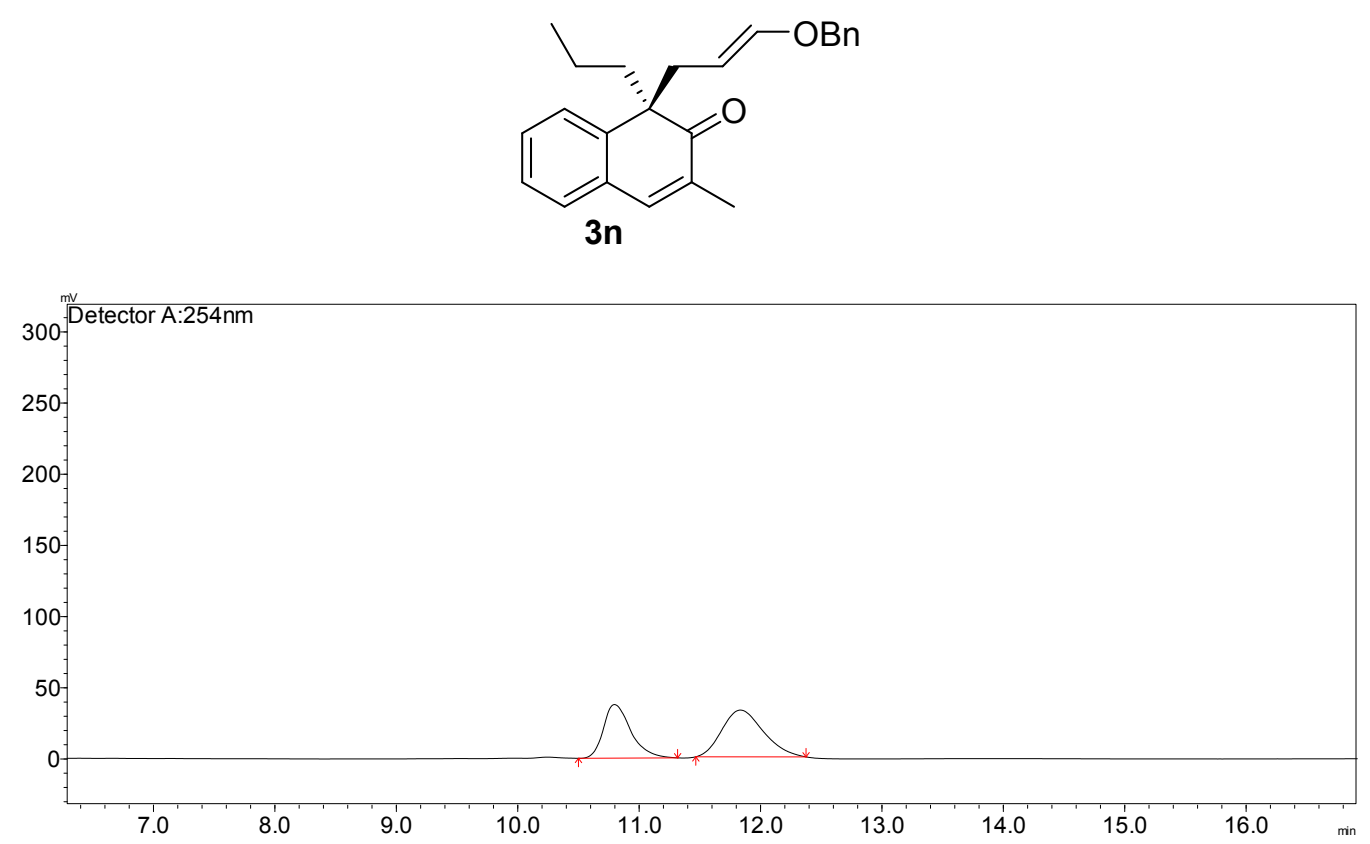

\begin{tabular}{|c|c|c|c|c|c|}
\hline Peak\# & Ret. Time & Area & Height & Area \% & Height \% \\
\hline 1 & 10.793 & 591570 & 37630 & 49.97 & 56.585 \\
\hline 2 & 11.83 & 592291 & 28872 & 50.03 & 43.415 \\
\hline Total & & 1183861 & 66503 & 100 & 100 \\
\hline
\end{tabular}

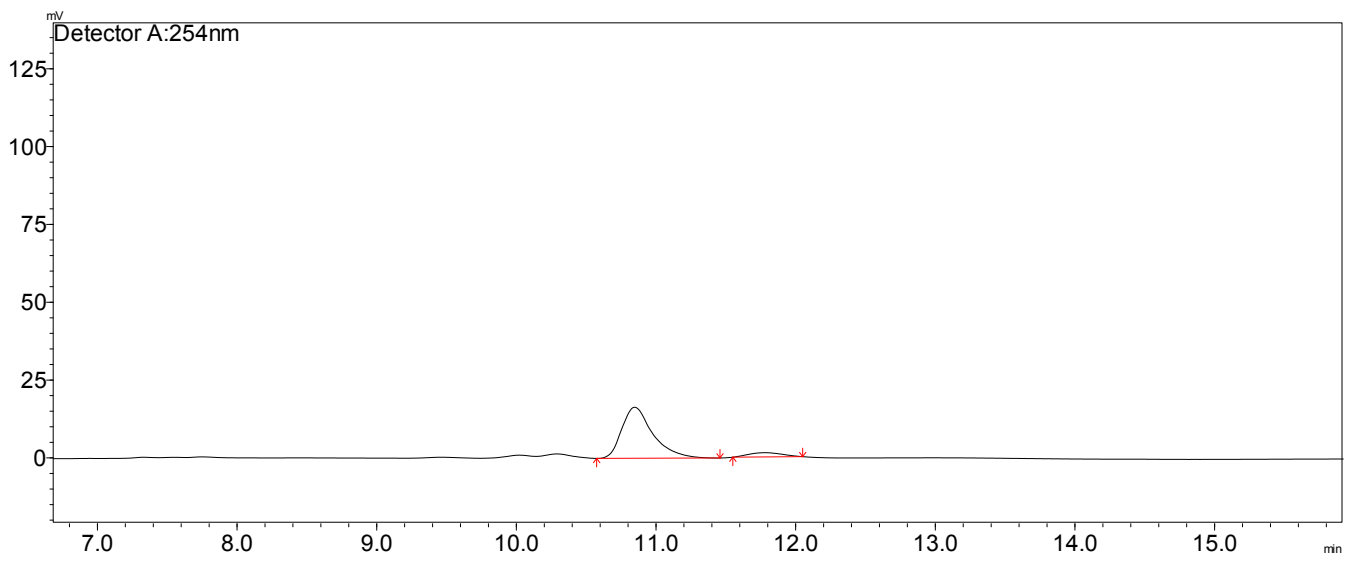

\begin{tabular}{|c|c|c|c|c|c|}
\hline Peak\# & Ret. Time & Area & Height & Area \% & Height \% \\
\hline 1 & 10.842 & 253268 & 16386 & 91.676 & 92.276 \\
\hline 2 & 11.773 & 22995 & 1372 & 8.324 & 7.724 \\
\hline Total & & 276263 & 17757 & 100 & 100 \\
\hline
\end{tabular}



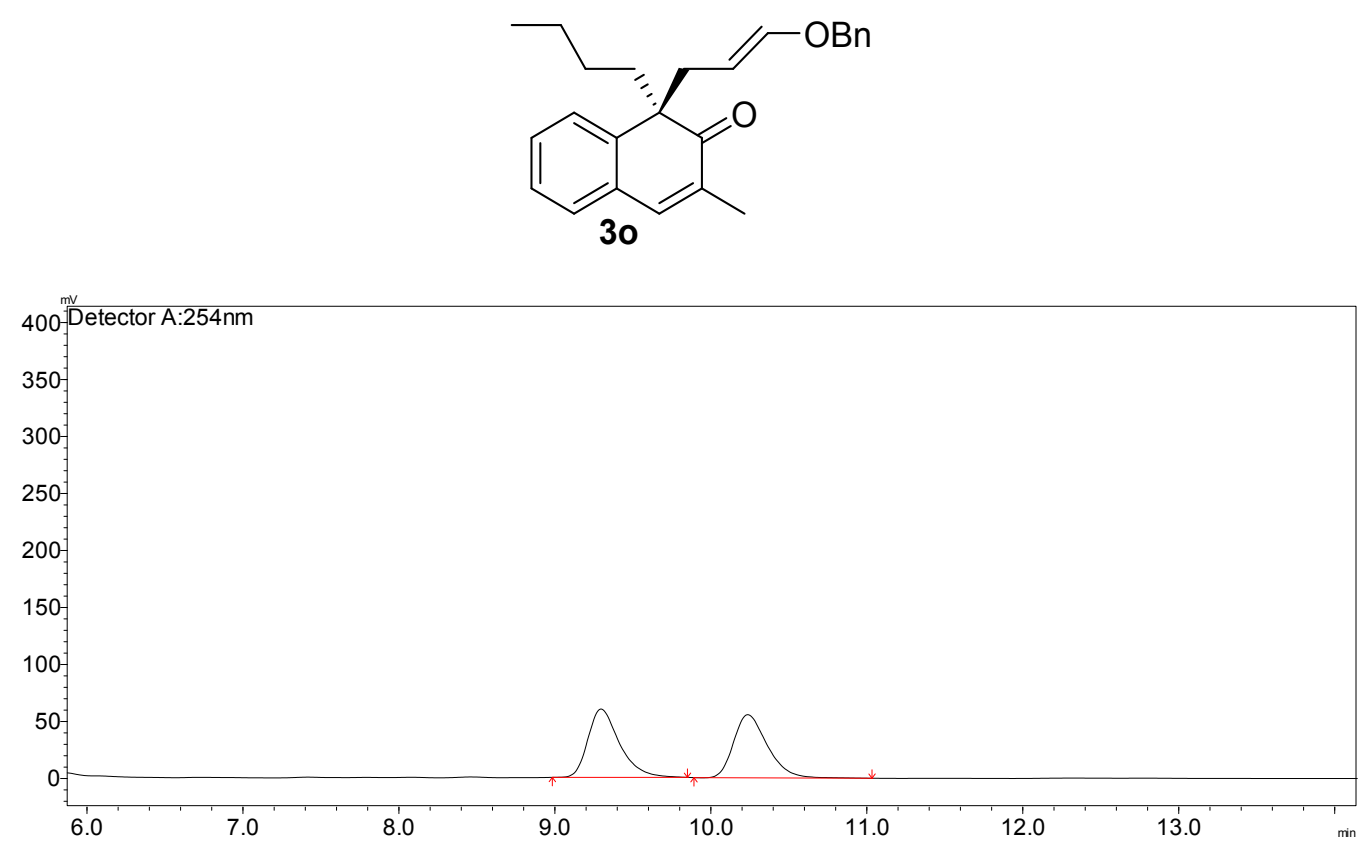

\begin{tabular}{|c|c|c|c|c|c|}
\hline Peak\# & Ret. Time & Area & Height & Area \% & Height \% \\
\hline 1 & 9.291 & 843785 & 59877 & 50.389 & 51.952 \\
\hline 2 & 10.232 & 830752 & 55378 & 49.611 & 48.048 \\
\hline Total & & 1674537 & 115255 & 100 & 100 \\
\hline
\end{tabular}

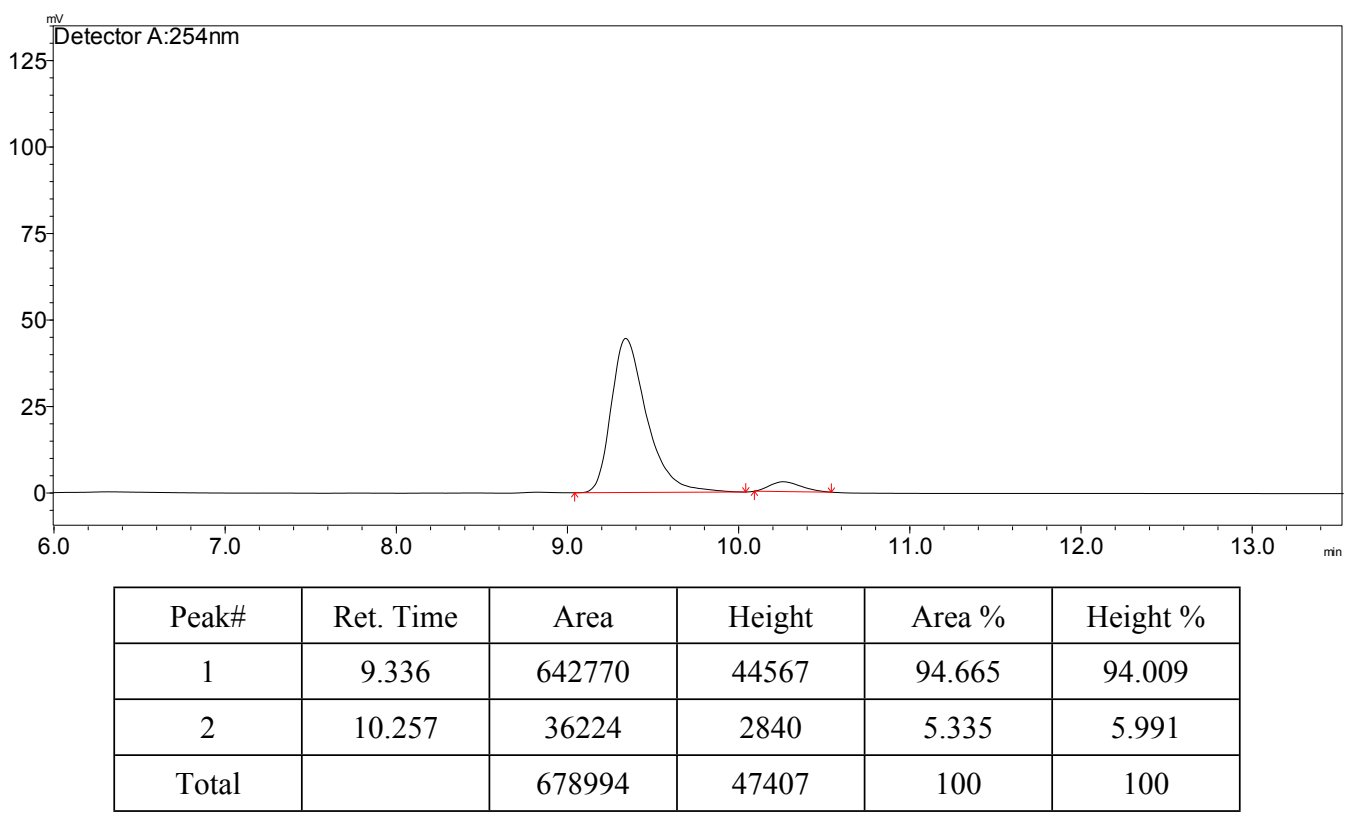



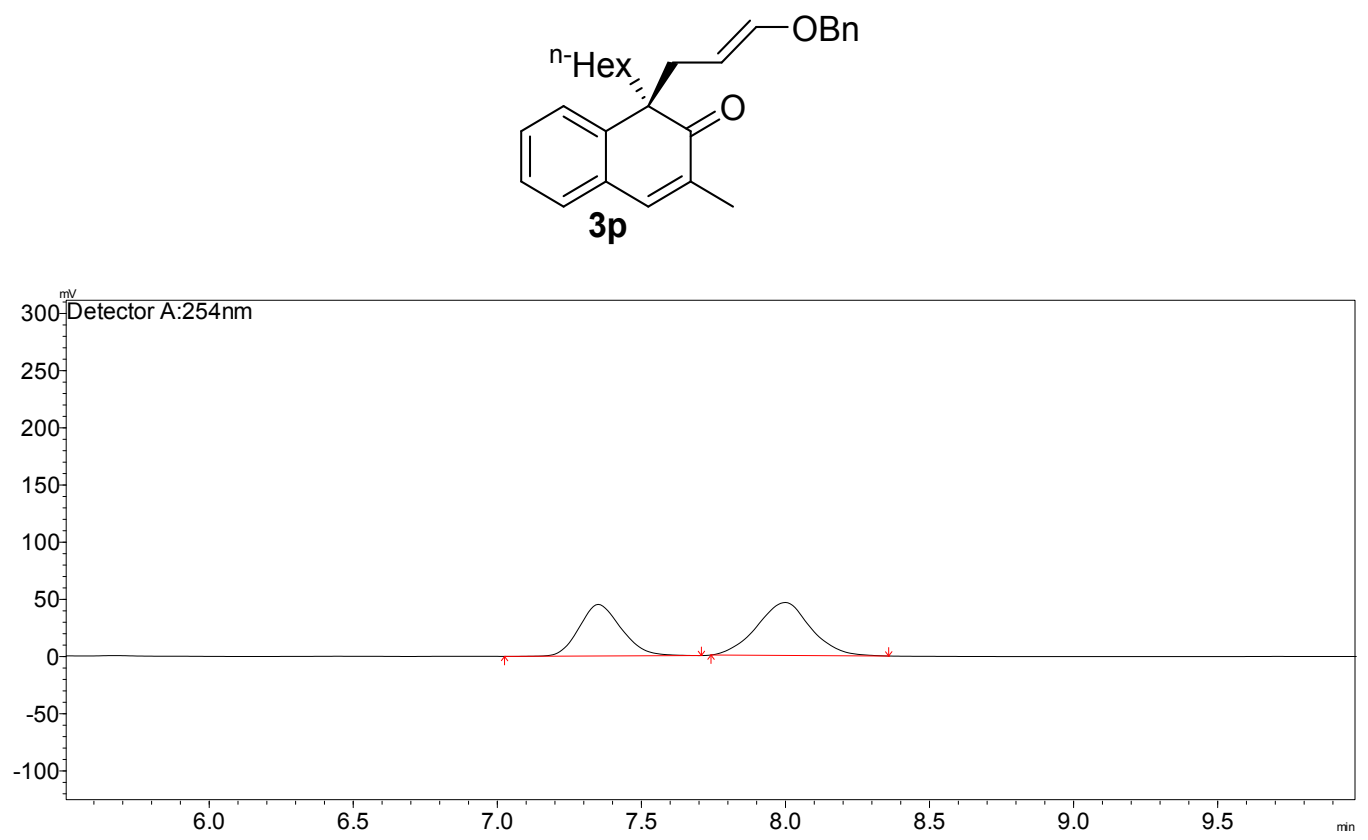

\begin{tabular}{|c|c|c|c|c|c|}
\hline Peak\# & Ret. Time & Area & Height & Area \% & Height \% \\
\hline 1 & 7.346 & 457201 & 45152 & 49.417 & 52.221 \\
\hline 2 & 7.995 & 467992 & 41311 & 50.583 & 47.779 \\
\hline Total & & 925193 & 86463 & 100 & 100 \\
\hline
\end{tabular}

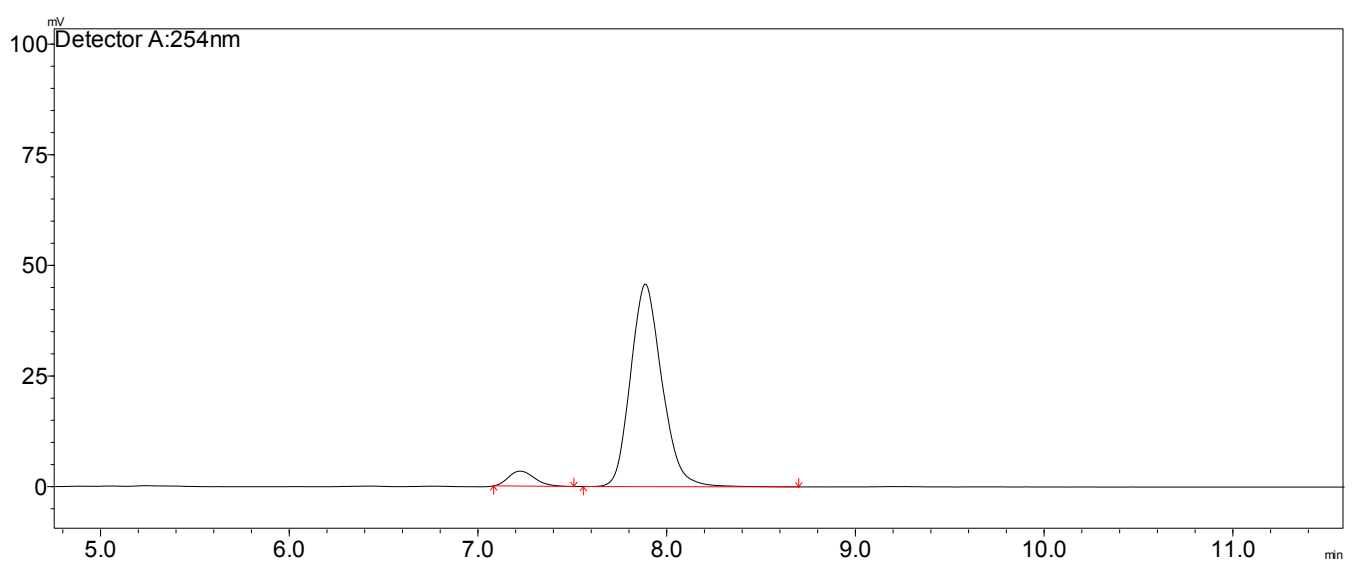

\begin{tabular}{|c|c|c|c|c|c|}
\hline Peak\# & Ret. Time & Area & Height & Area \% & Height \% \\
\hline 1 & 7.218 & 32459 & 3366 & 5.792 & 6.85 \\
\hline 2 & 7.883 & 527903 & 45769 & 94.208 & 93.15 \\
\hline Total & & 560361 & 49134 & 100 & 100 \\
\hline
\end{tabular}




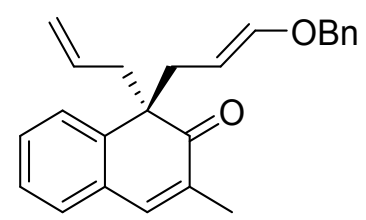

$3 q$

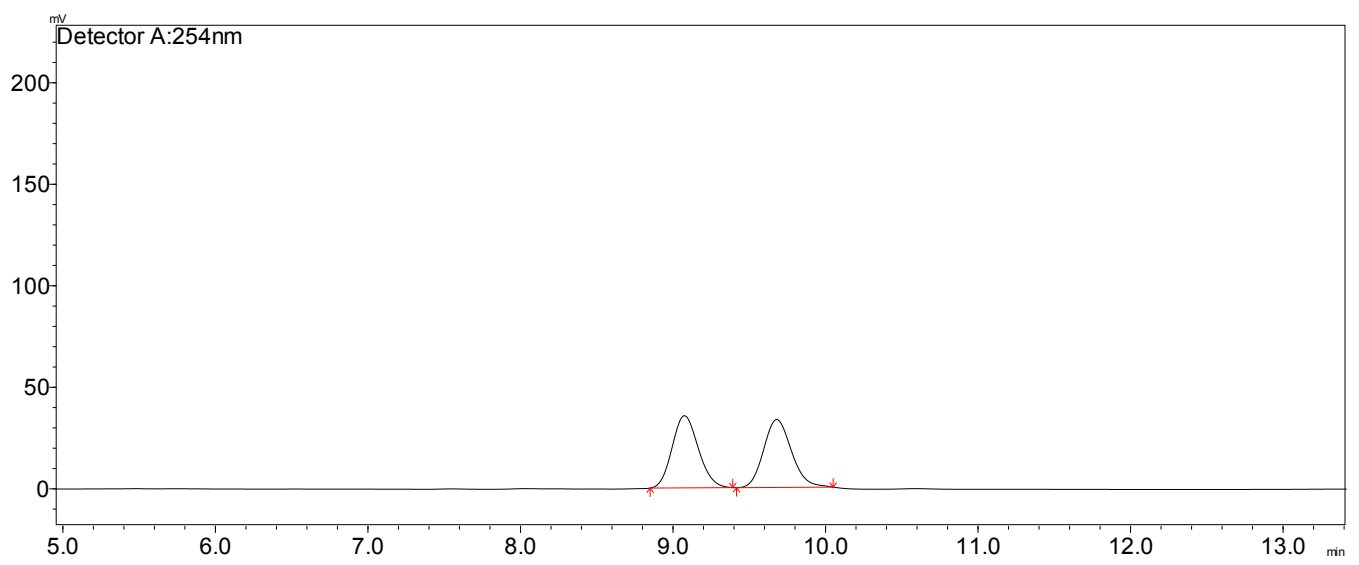

\begin{tabular}{|c|c|c|c|c|c|}
\hline Peak\# & Ret. Time & Area & Height & Area \% & Height \% \\
\hline 1 & 9.071 & 425996 & 35584 & 50.05 & 51.543 \\
\hline 2 & 9.676 & 425141 & 33454 & 49.95 & 48.457 \\
\hline Total & & 851138 & 69038 & 100 & 100 \\
\hline
\end{tabular}

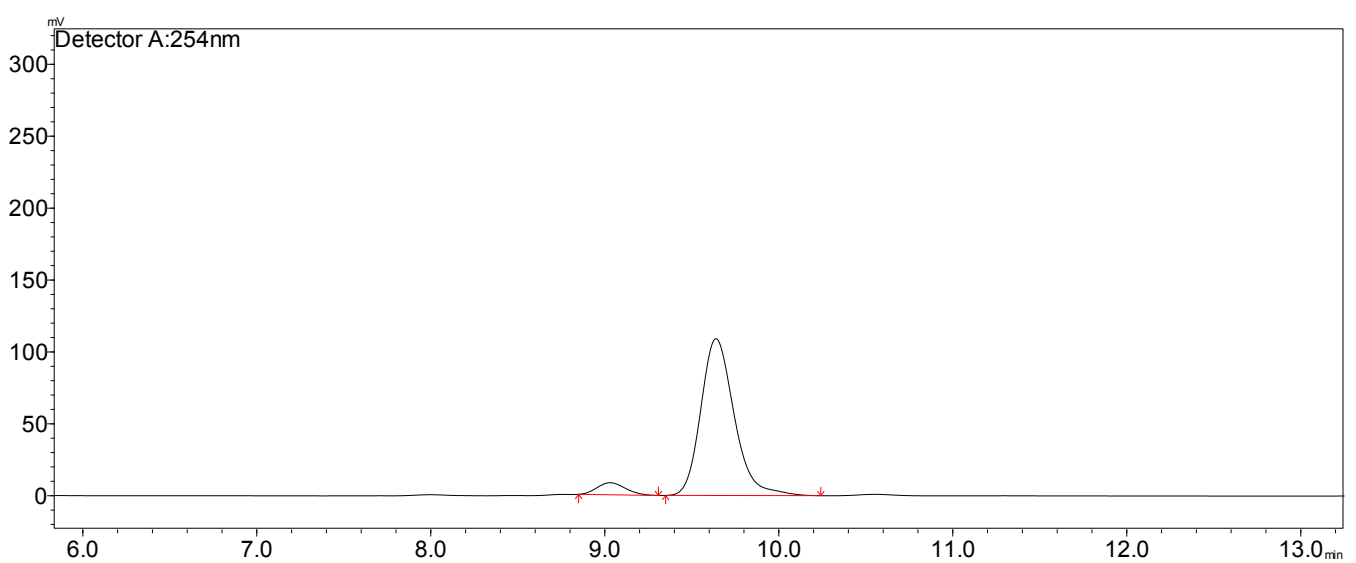

\begin{tabular}{|c|c|c|c|c|c|}
\hline Peak\# & Ret. Time & Area & Height & Area \% & Height \% \\
\hline 1 & 9.026 & 94770 & 8360 & 6.116 & 7.117 \\
\hline 2 & 9.635 & 1454865 & 109116 & 93.884 & 92.883 \\
\hline Total & & 1549635 & 117477 & 100 & 100 \\
\hline
\end{tabular}



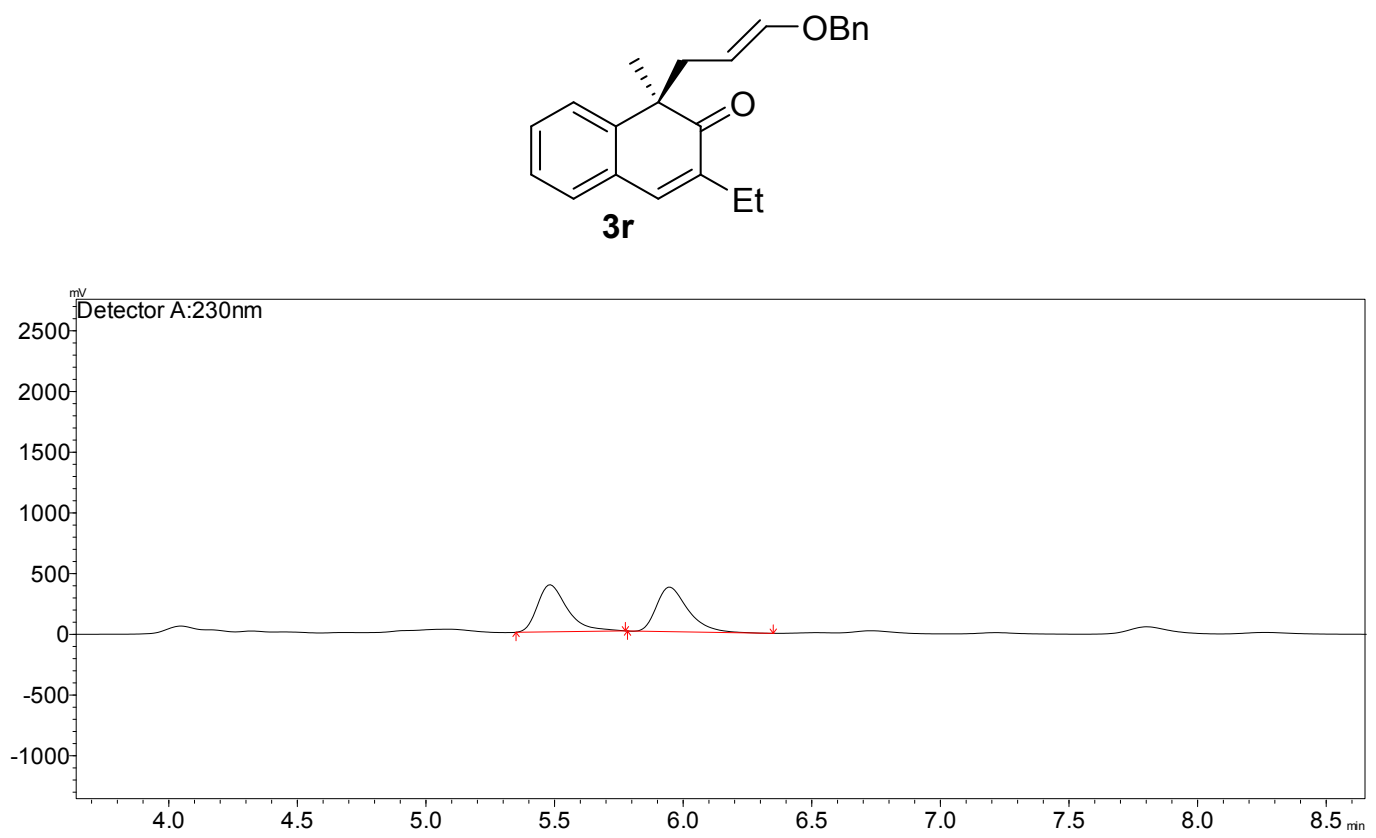

\begin{tabular}{|c|c|c|c|c|c|}
\hline Peak\# & Ret. Time & Area & Height & Area \% & Height \% \\
\hline 1 & 5.477 & 3130316 & 387655 & 49.98 & 51.412 \\
\hline 2 & 5.942 & 3132877 & 366360 & 50.02 & 48.588 \\
\hline Total & & 6263192 & 754015 & 100 & 100 \\
\hline
\end{tabular}

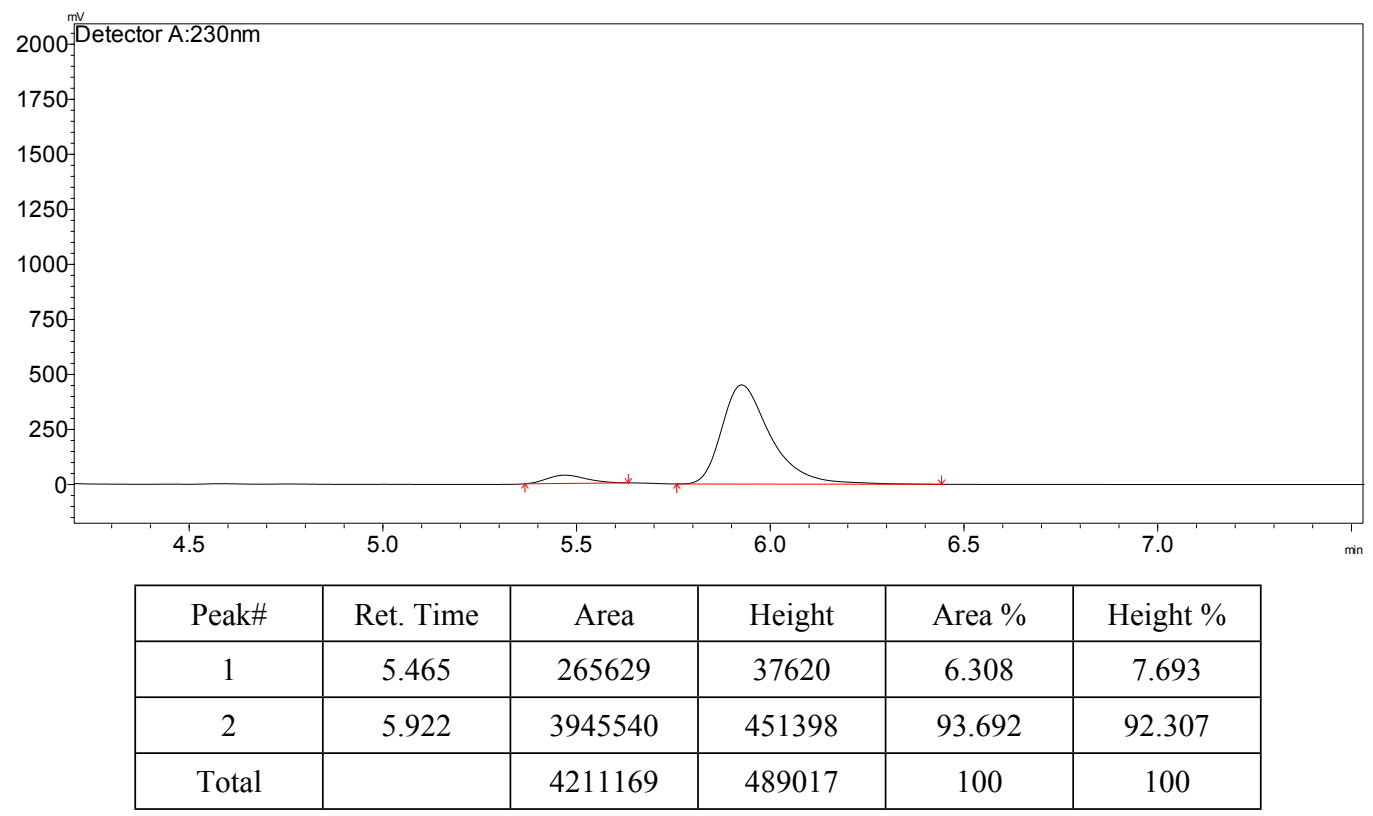



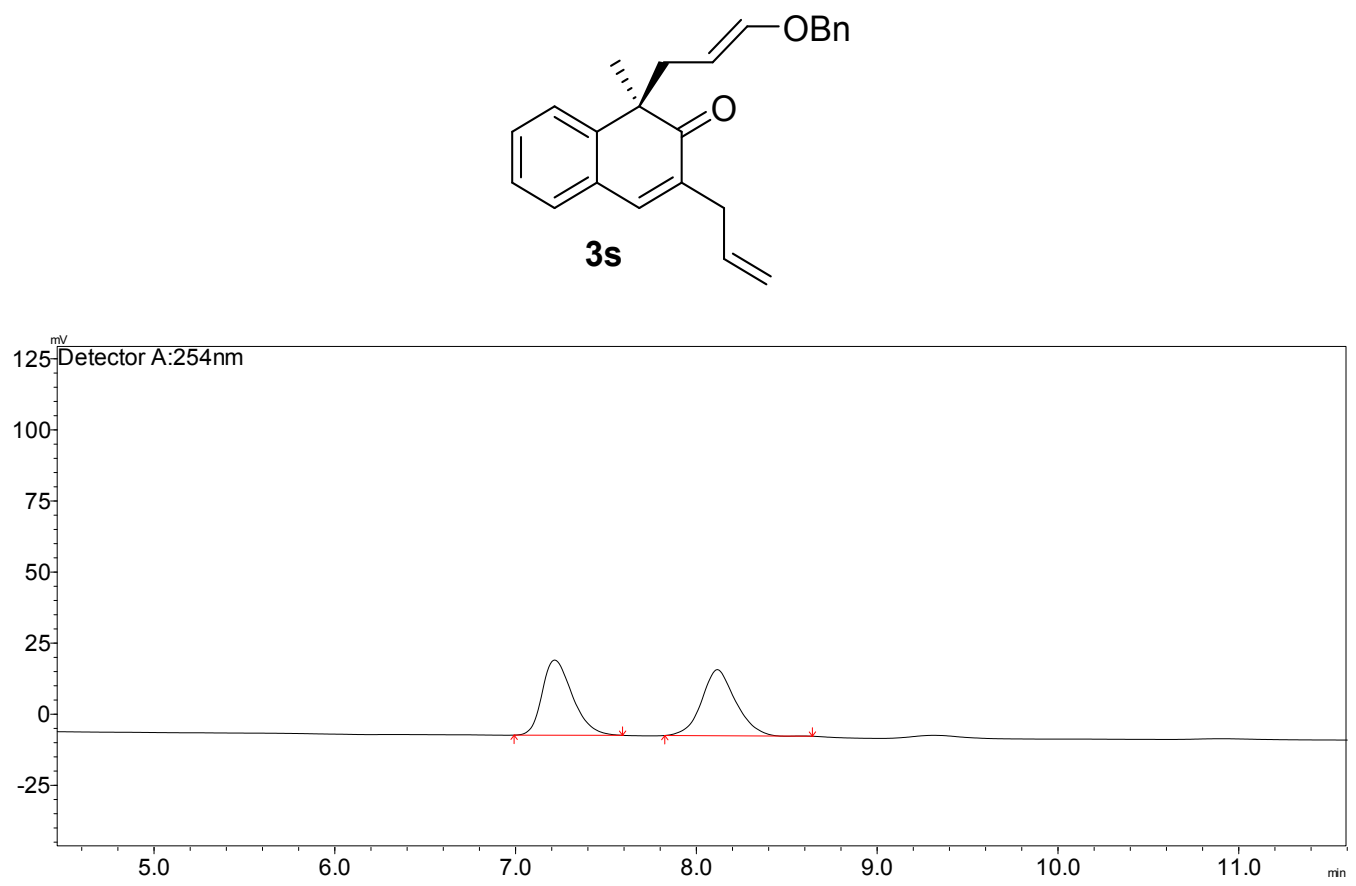

\begin{tabular}{|c|c|c|c|c|c|}
\hline Peak\# & Ret. Time & Area & Height & Area \% & Height \% \\
\hline 1 & 7.212 & 308088 & 26449 & 50.27 & 53.195 \\
\hline 2 & 8.112 & 304777 & 23272 & 49.73 & 46.805 \\
\hline Total & & 612865 & 49721 & 100 & 100 \\
\hline
\end{tabular}

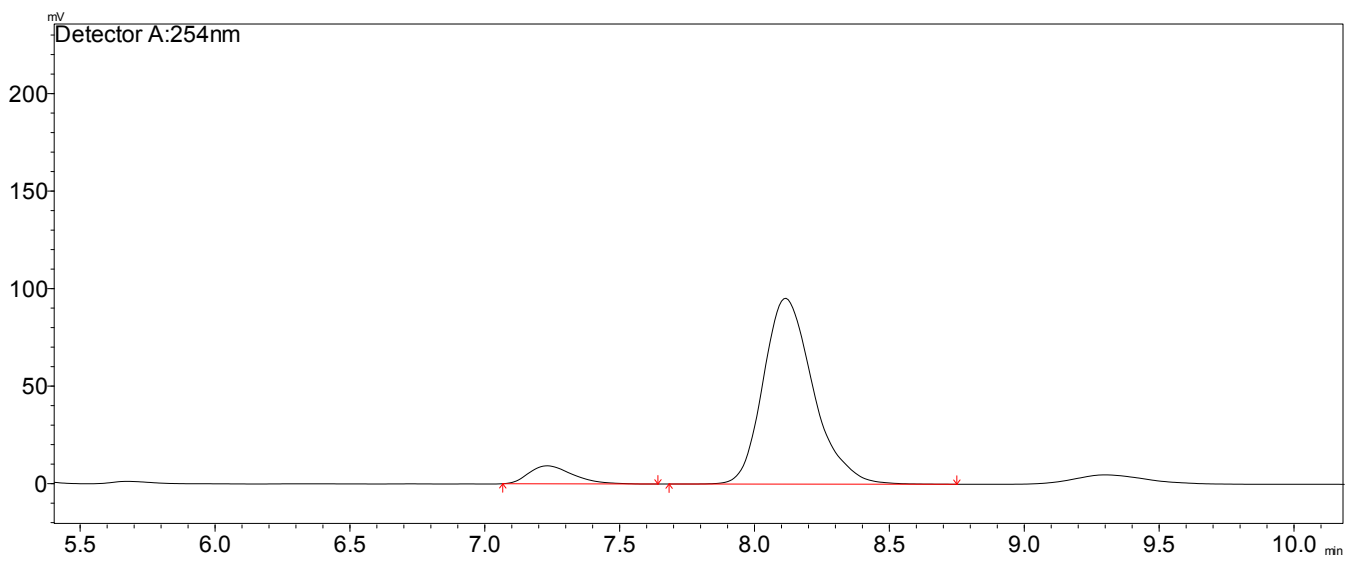

\begin{tabular}{|c|c|c|c|c|c|}
\hline Peak\# & Ret. Time & Area & Height & Area \% & Height \% \\
\hline 1 & 7.227 & 104404 & 9234 & 7.845 & 8.836 \\
\hline 2 & 8.11 & 1226410 & 95265 & 92.155 & 91.164 \\
\hline Total & & 1330813 & 104499 & 100 & 100 \\
\hline
\end{tabular}



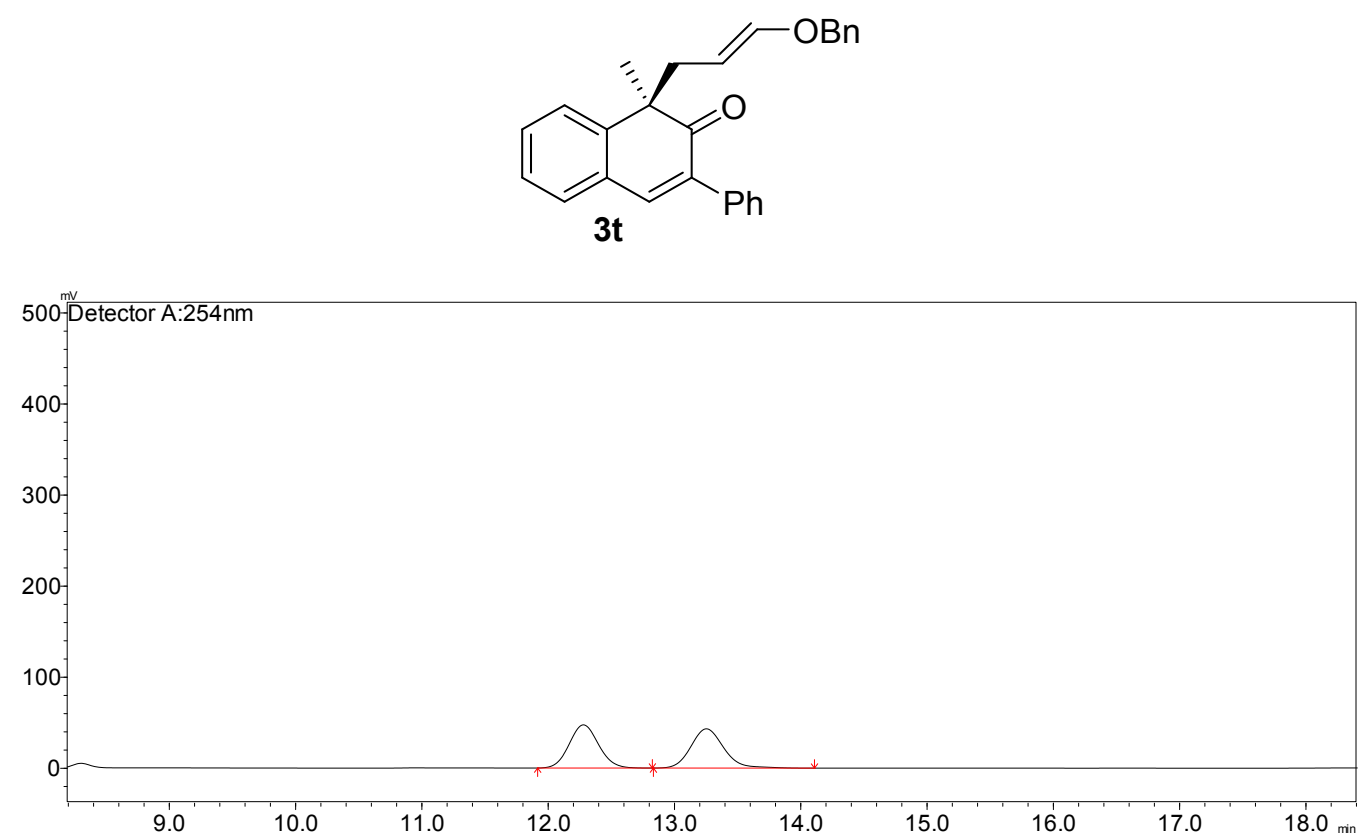

\begin{tabular}{|c|c|c|c|c|c|}
\hline Peak\# & Ret. Time & Area & Height & Area \% & Height \% \\
\hline 1 & 12.274 & 767534 & 47375 & 49.655 & 52.351 \\
\hline 2 & 13.248 & 778187 & 43119 & 50.345 & 47.649 \\
\hline Total & & 1545722 & 90494 & 100 & 100 \\
\hline
\end{tabular}

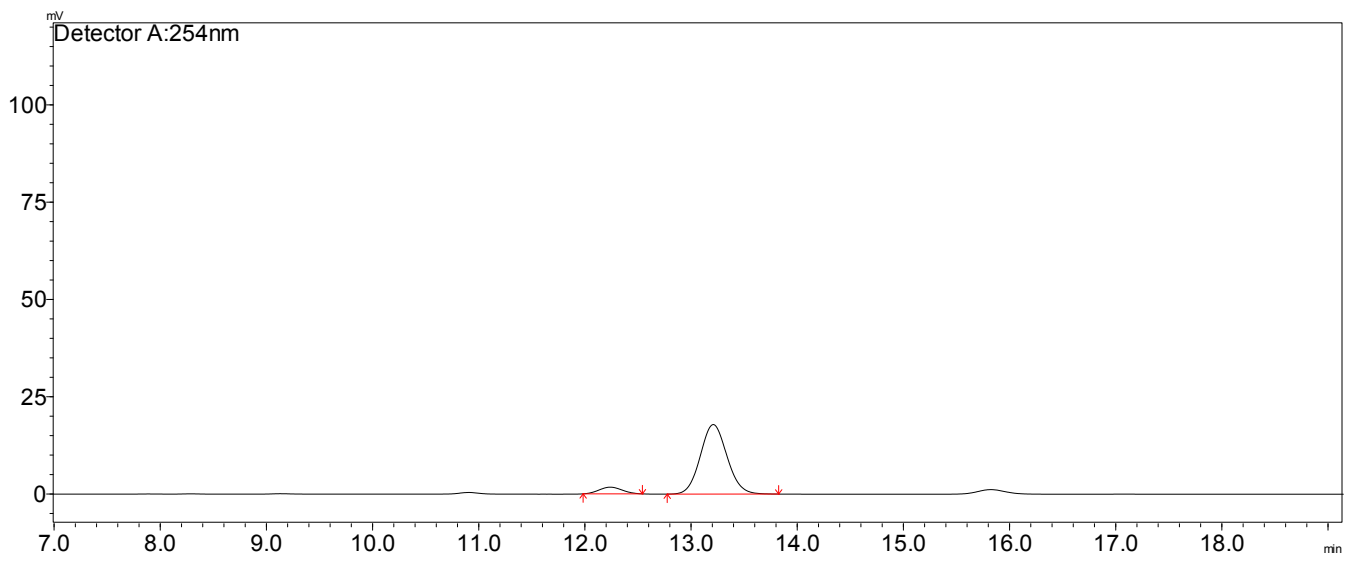

\begin{tabular}{|c|c|c|c|c|c|}
\hline Peak\# & Ret. Time & Area & Height & Area \% & Height \% \\
\hline 1 & 12.235 & 26122 & 1717 & 7.699 & 8.762 \\
\hline 2 & 13.205 & 313158 & 17882 & 92.301 & 91.238 \\
\hline Total & & 339281 & 19599 & 100 & 100 \\
\hline
\end{tabular}



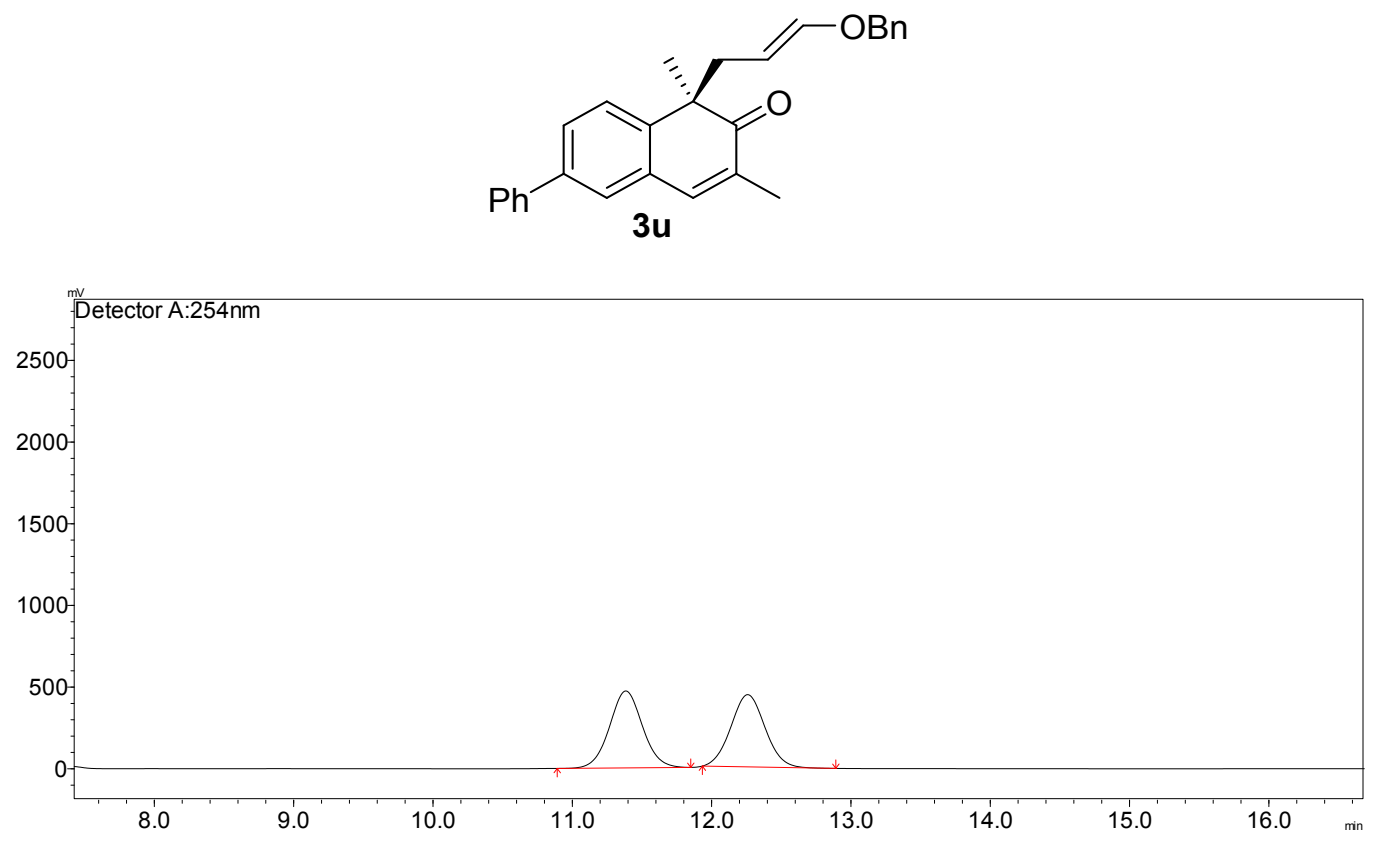

\begin{tabular}{|c|c|c|c|c|c|}
\hline Peak\# & Ret. Time & Area & Height & Area \% & Height \% \\
\hline 1 & 11.38 & 7757783 & 471265 & 50.732 & 51.591 \\
\hline 2 & 12.254 & 7533764 & 442203 & 49.268 & 48.409 \\
\hline Total & & 15291548 & 913468 & 100 & 100 \\
\hline
\end{tabular}

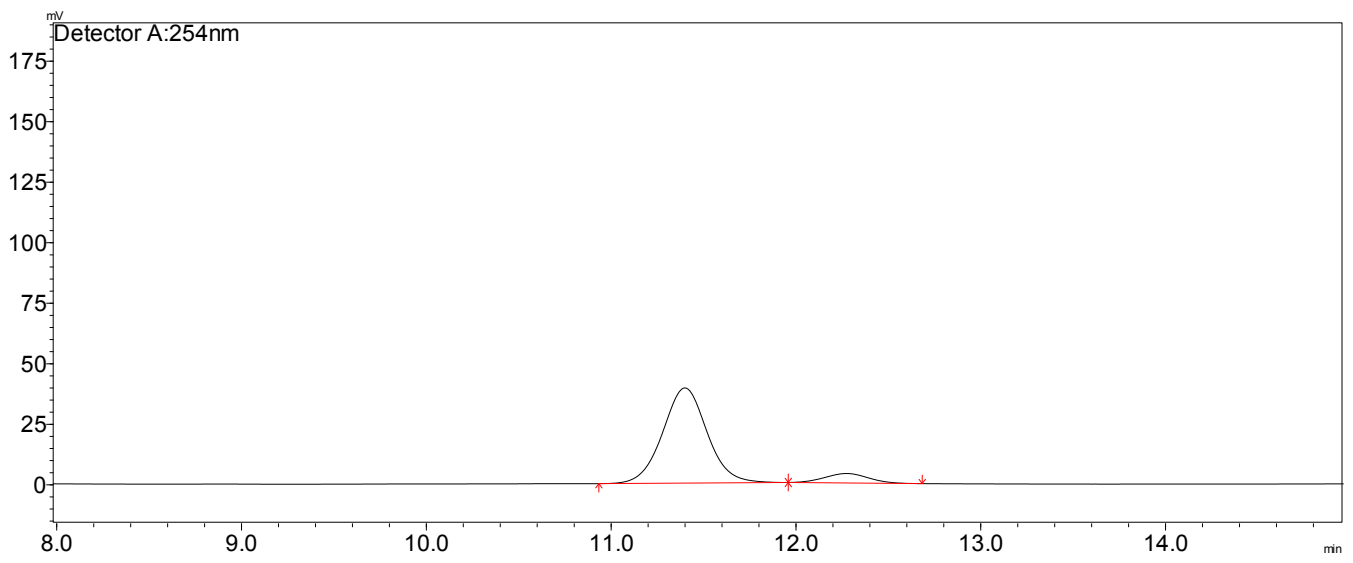

\begin{tabular}{|c|c|c|c|c|c|}
\hline Peak\# & Ret. Time & Area & Height & Area \% & Height \% \\
\hline 1 & 11.395 & 657434 & 39344 & 91.114 & 90.995 \\
\hline 2 & 12.27 & 64120 & 3893 & 8.886 & 9.005 \\
\hline Total & & 721554 & 43238 & 100 & 100 \\
\hline
\end{tabular}



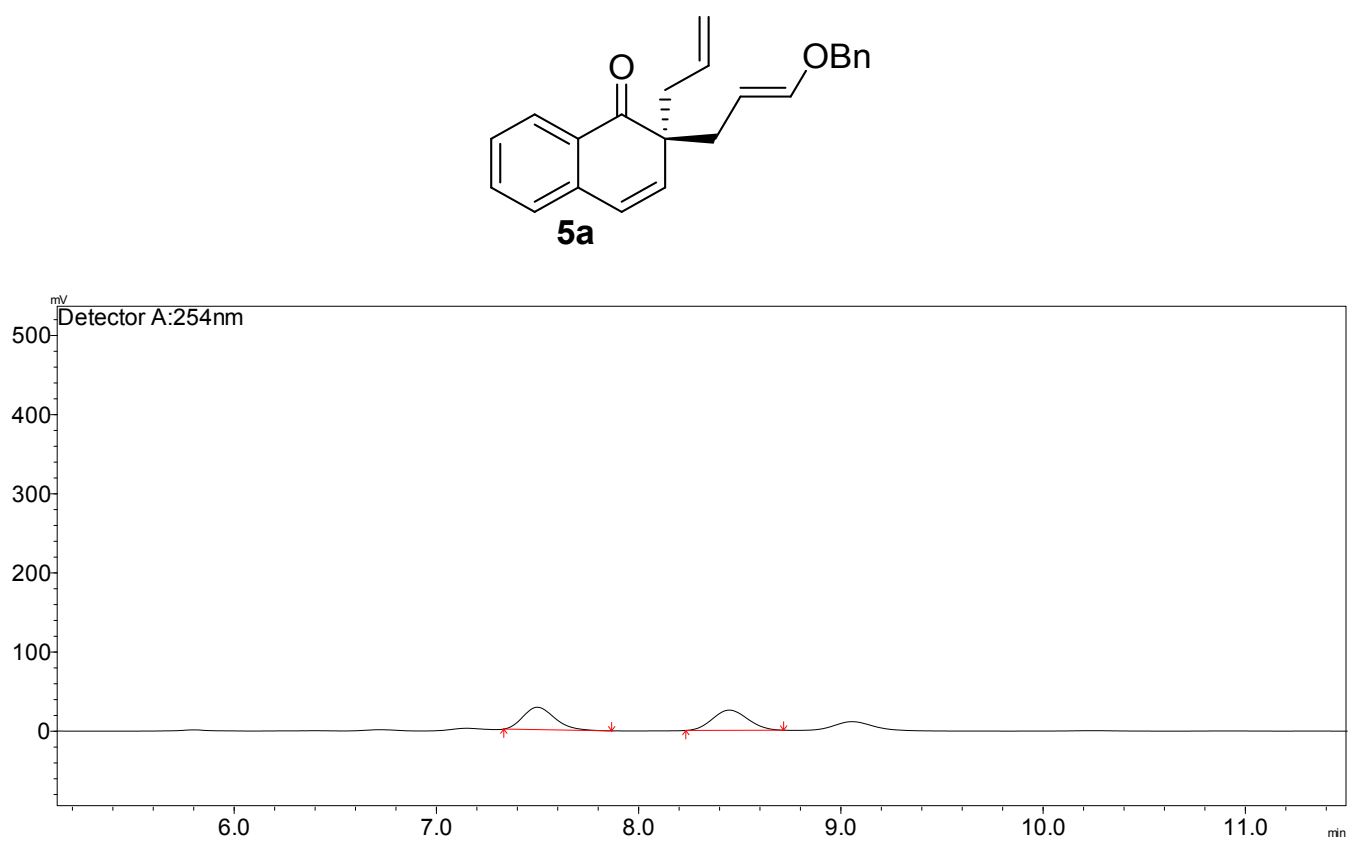

\begin{tabular}{|c|c|c|c|c|c|}
\hline Peak\# & Ret. Time & Area & Height & Area \% & Height \% \\
\hline 1 & 7.495 & 306197 & 28244 & 49.743 & 52.465 \\
\hline 2 & 8.444 & 309360 & 25590 & 50.257 & 47.535 \\
\hline Total & & 615557 & 53834 & 100 & 100 \\
\hline
\end{tabular}

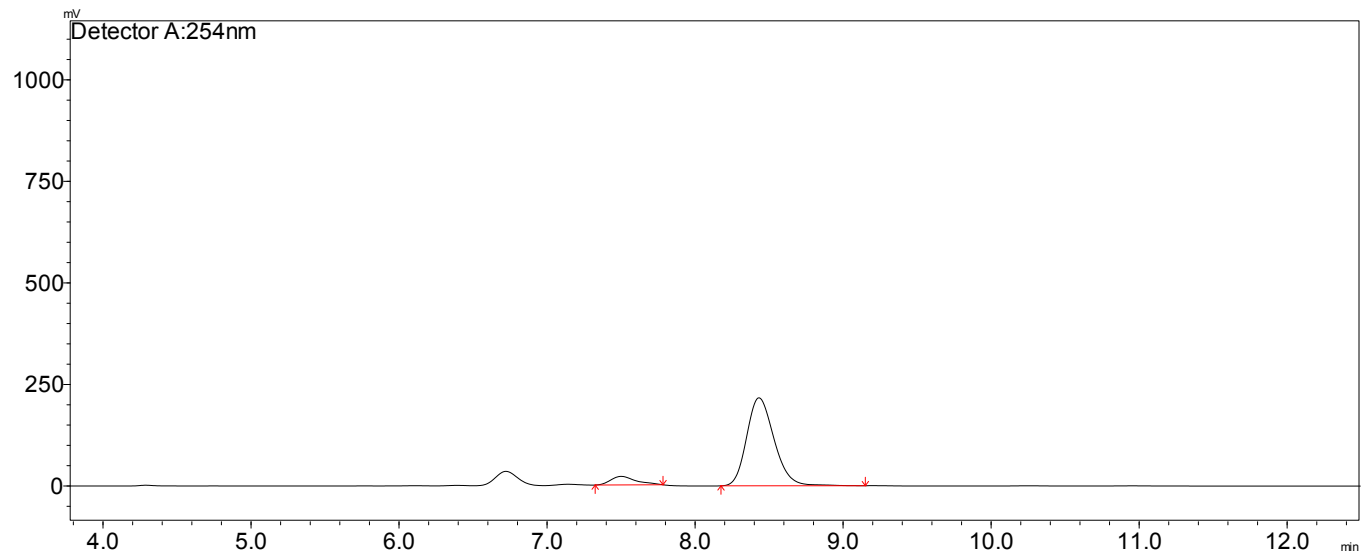

\begin{tabular}{|c|c|c|c|c|c|}
\hline Peak\# & Ret. Time & Area & Height & Area \% & Height $\%$ \\
\hline 1 & 7.497 & 252430 & 21149 & 8.289 & 8.892 \\
\hline 2 & 8.427 & 2793068 & 216708 & 91.711 & 91.108 \\
\hline Total & & 3045498 & 237857 & 100 & 100 \\
\hline
\end{tabular}



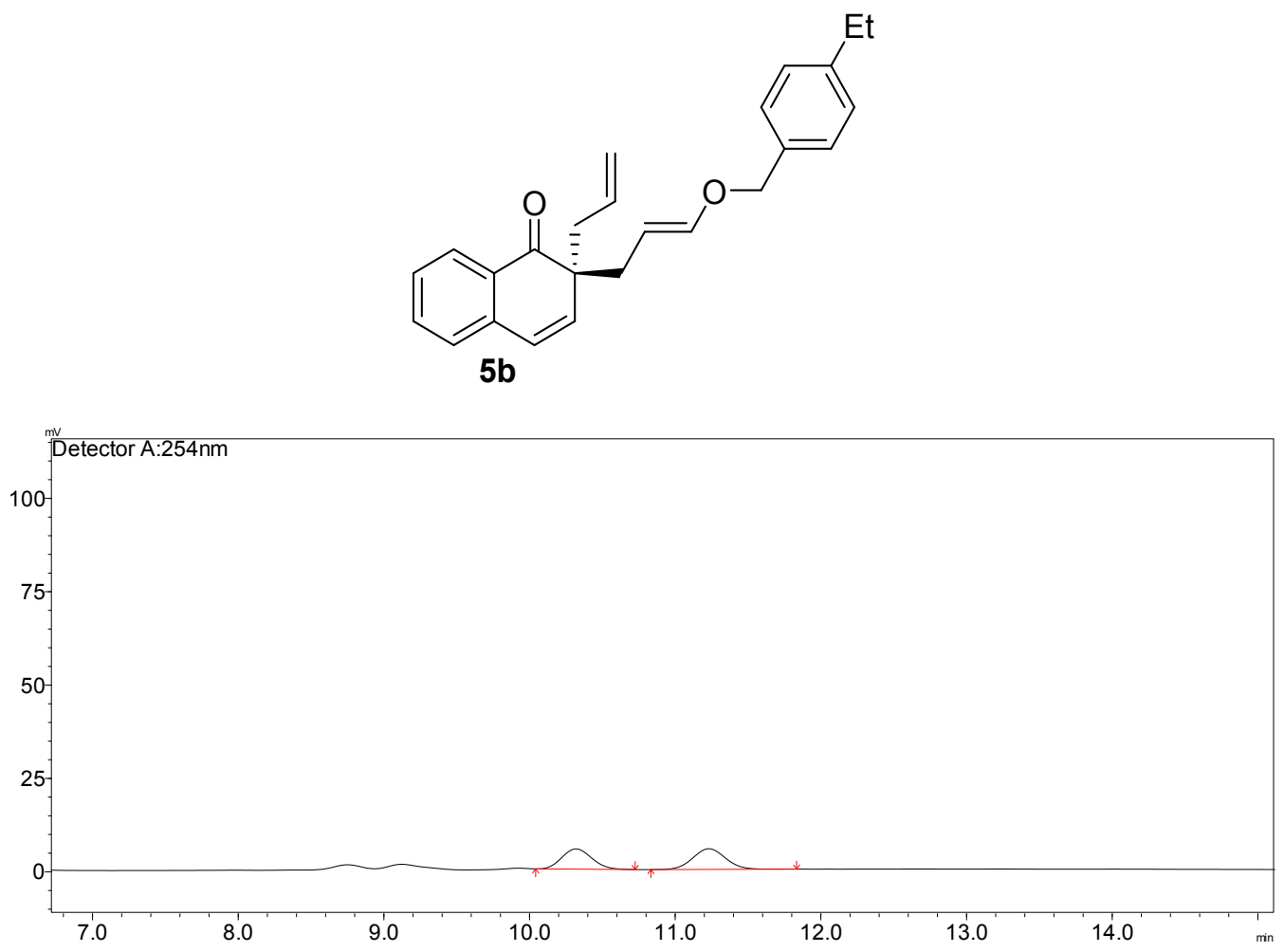

\begin{tabular}{|c|c|c|c|c|c|}
\hline Peak\# & Ret. Time & Area & Height & Area \% & Height \% \\
\hline 1 & 10.314 & 77427 & 5434 & 47.131 & 49.494 \\
\hline 2 & 11.227 & 86855 & 5545 & 52.869 & 50.506 \\
\hline Total & & 164282 & 10978 & 100 & 100 \\
\hline
\end{tabular}

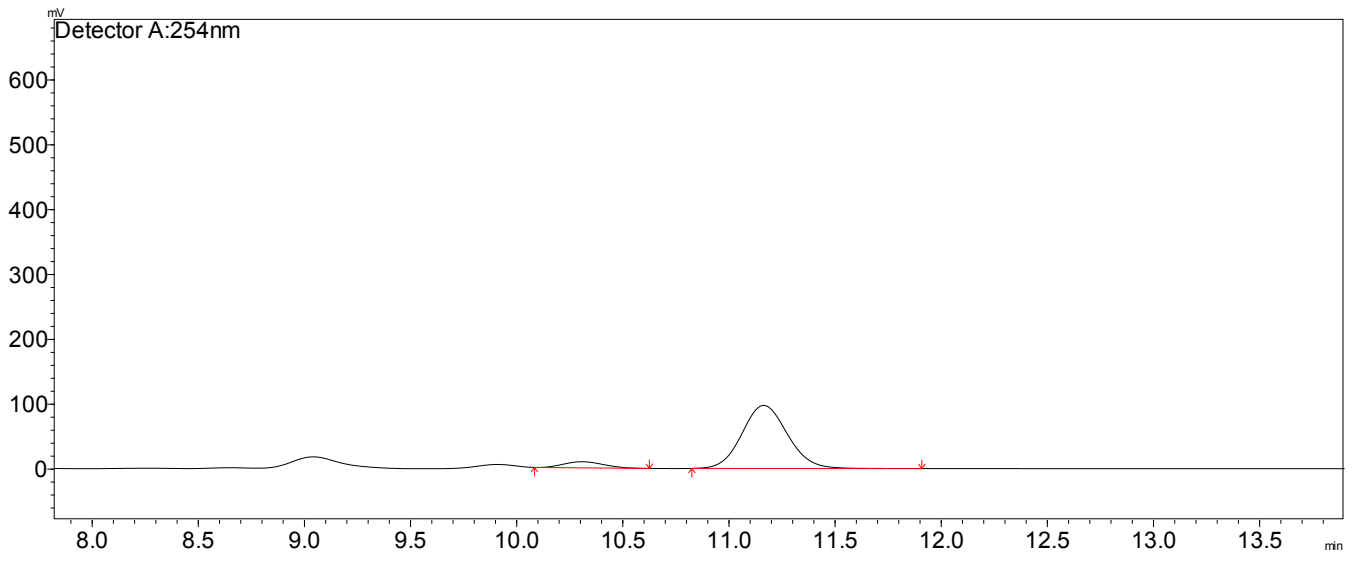

\begin{tabular}{|c|c|c|c|c|c|}
\hline Peak\# & Ret. Time & Area & Height & Area \% & Height \% \\
\hline 1 & 10.303 & 123727 & 9447 & 7.85 & 8.853 \\
\hline 2 & 11.159 & 1452485 & 97267 & 92.15 & 91.147 \\
\hline Total & & 1576212 & 106714 & 100 & 100 \\
\hline
\end{tabular}



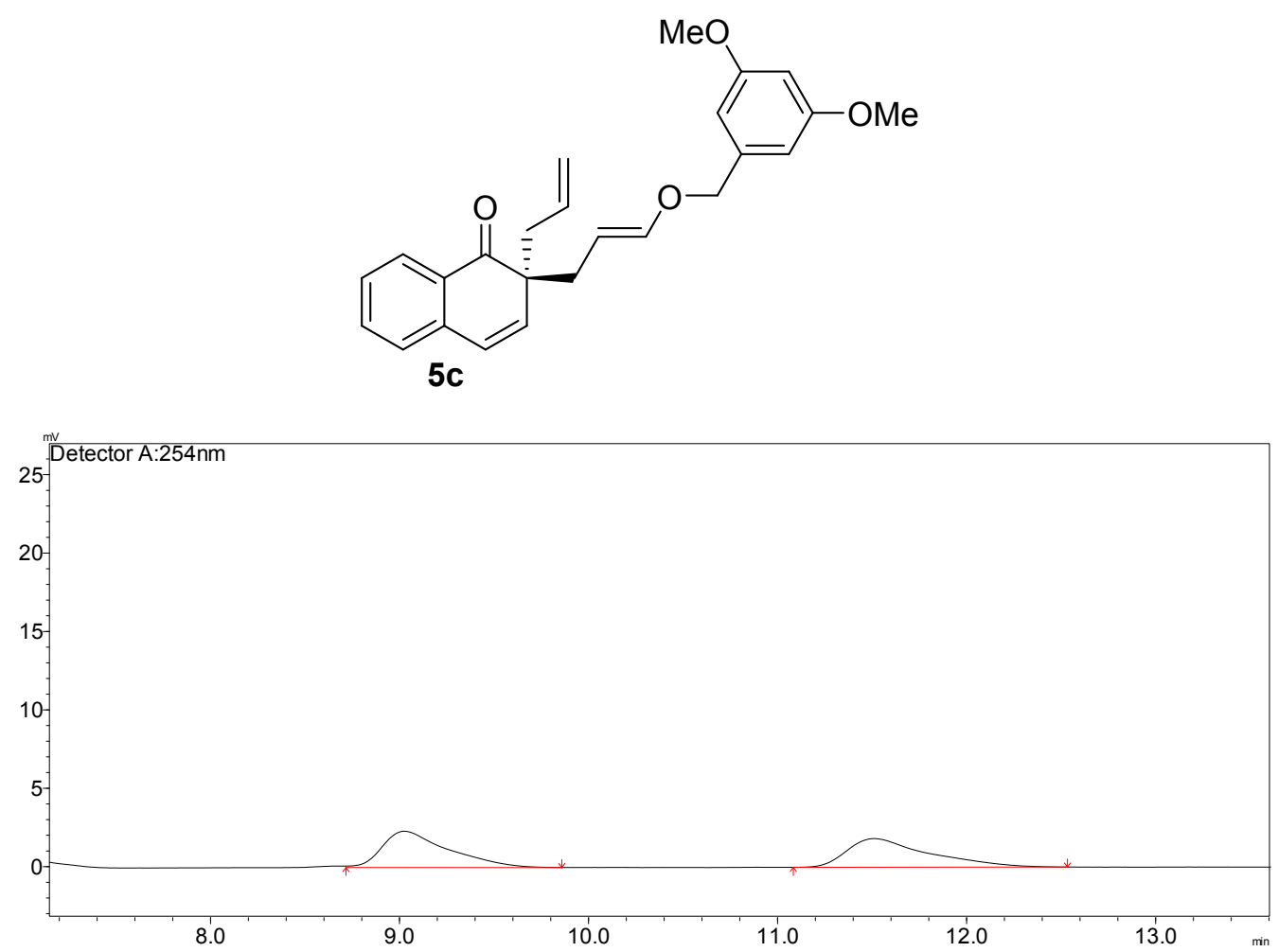

\begin{tabular}{|c|c|c|c|c|c|}
\hline Peak\# & Ret. Time & Area & Height & Area \% & Height \% \\
\hline 1 & 9.021 & 56076 & 2314 & 50.689 & 55.901 \\
\hline 2 & 11.506 & 54552 & 1825 & 49.311 & 44.099 \\
\hline Total & & 110628 & 4139 & 100 & 100 \\
\hline
\end{tabular}

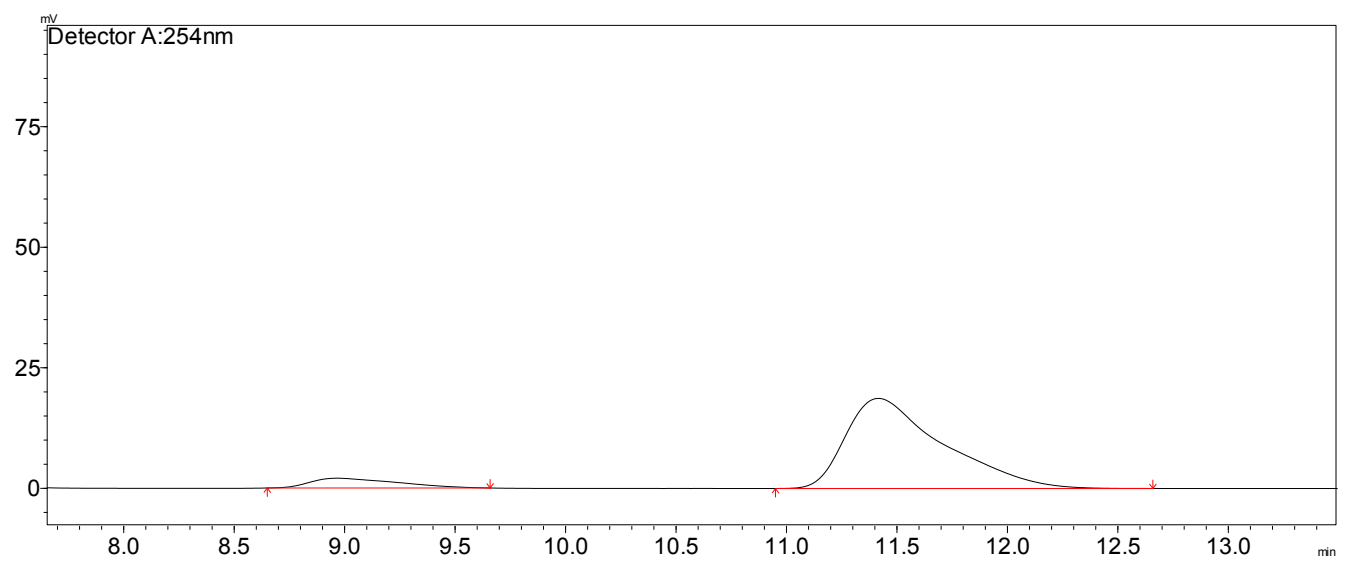

\begin{tabular}{|c|c|c|c|c|c|}
\hline Peak\# & Ret. Time & Area & Height & Area \% & Height \% \\
\hline 1 & 8.961 & 54797 & 2030 & 8.781 & 9.798 \\
\hline 2 & 11.412 & 569249 & 18687 & 91.219 & 90.202 \\
\hline Total & & 624046 & 20717 & 100 & 100 \\
\hline
\end{tabular}



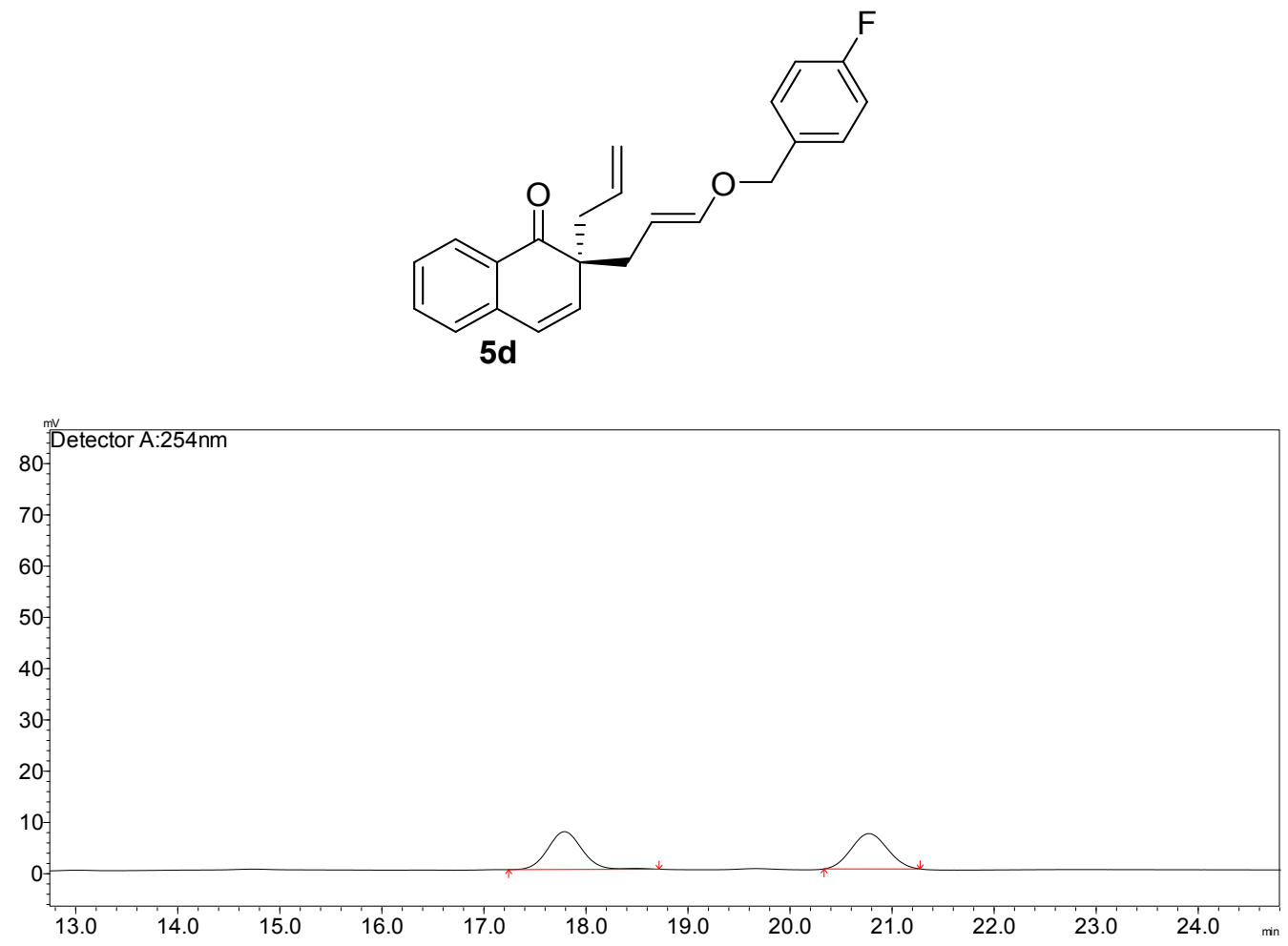

\begin{tabular}{|c|c|c|c|c|c|}
\hline Peak\# & Ret. Time & Area & Height & Area \% & Height \% \\
\hline 1 & 17.785 & 172522 & 7380 & 50.227 & 51.711 \\
\hline 2 & 20.769 & 170960 & 6892 & 49.773 & 48.289 \\
\hline Total & & 343481 & 14272 & 100 & 100 \\
\hline
\end{tabular}

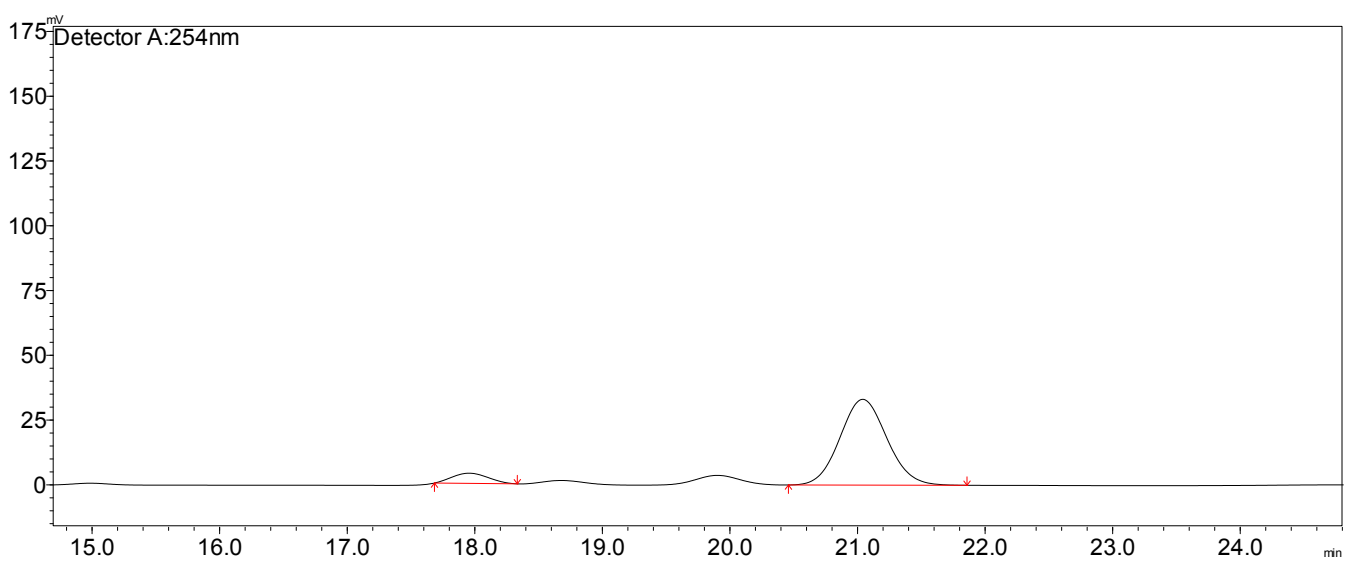

\begin{tabular}{|c|c|c|c|c|c|}
\hline Peak\# & Ret. Time & Area & Height & Area \% & Height \% \\
\hline 1 & 17.952 & 77290 & 3935 & 8.352 & 10.621 \\
\hline 2 & 21.036 & 848165 & 33113 & 91.648 & 89.379 \\
\hline Total & & 925455 & 37048 & 100 & 100 \\
\hline
\end{tabular}



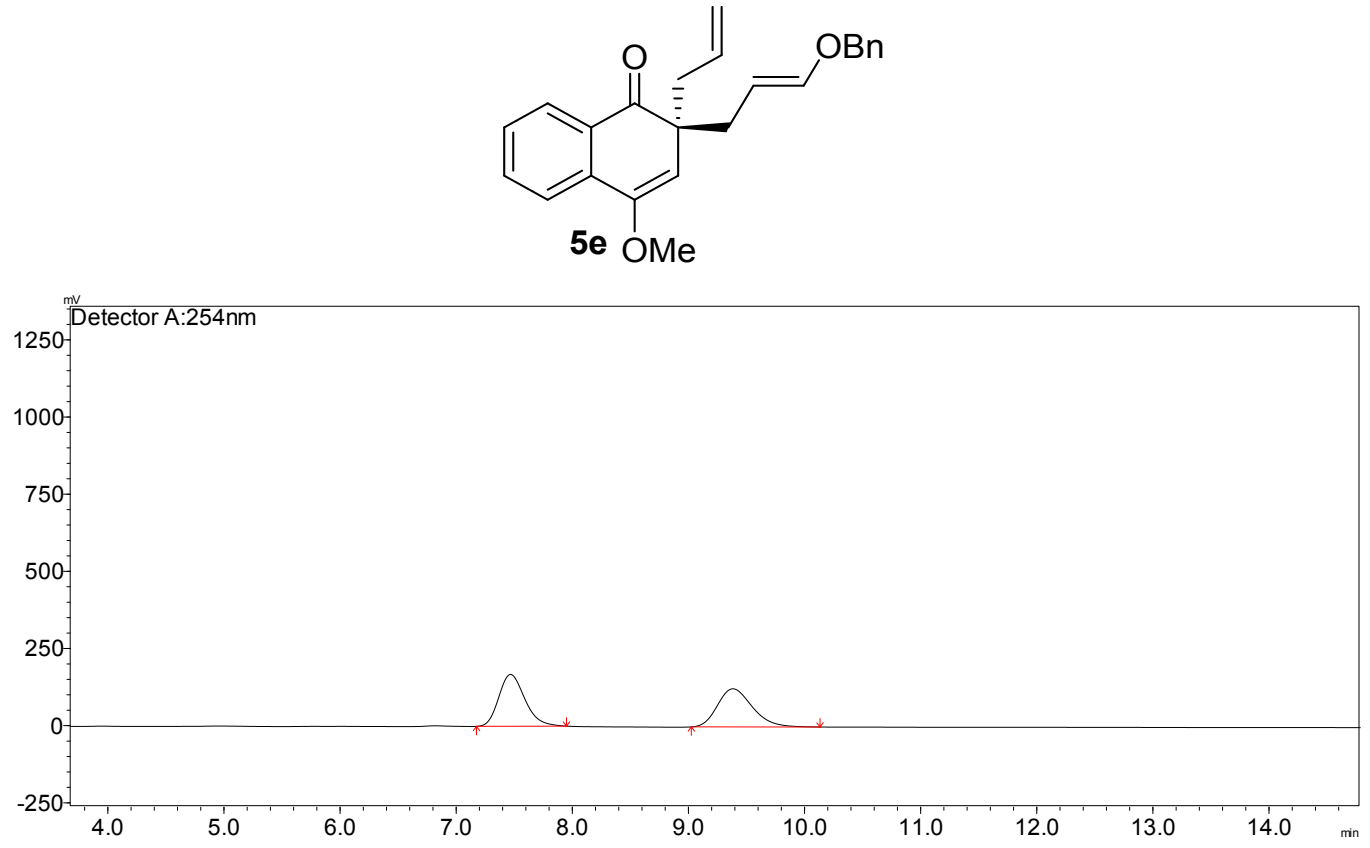

\begin{tabular}{|c|c|c|c|c|c|}
\hline Peak\# & Ret. Time & Area & Height & Area \% & Height \% \\
\hline 1 & 7.464 & 2620590 & 168007 & 50.435 & 57.625 \\
\hline 2 & 9.379 & 2575356 & 123546 & 49.565 & 42.375 \\
\hline Total & & 5195946 & 291553 & 100 & 100 \\
\hline
\end{tabular}

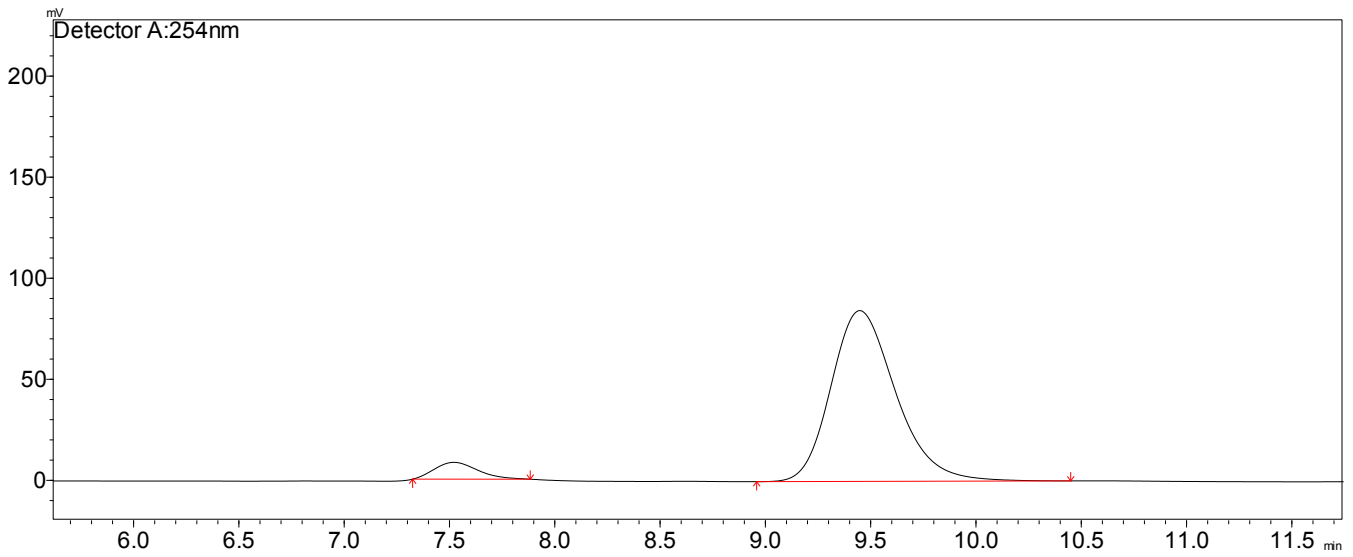

\begin{tabular}{|c|c|c|c|c|c|}
\hline Peak\# & Ret. Time & Area & Height & Area \% & Height \% \\
\hline 1 & 7.516 & 119562 & 8319 & 6.212 & 8.959 \\
\hline 2 & 9.444 & 1805171 & 84537 & 93.788 & 91.041 \\
\hline Total & & 1924733 & 92856 & 100 & 100 \\
\hline
\end{tabular}<smiles>C=CC[C@]1(C/C=C/OCc2ccccc2)C=C(OCC)c2ccccc2C1=O</smiles> 


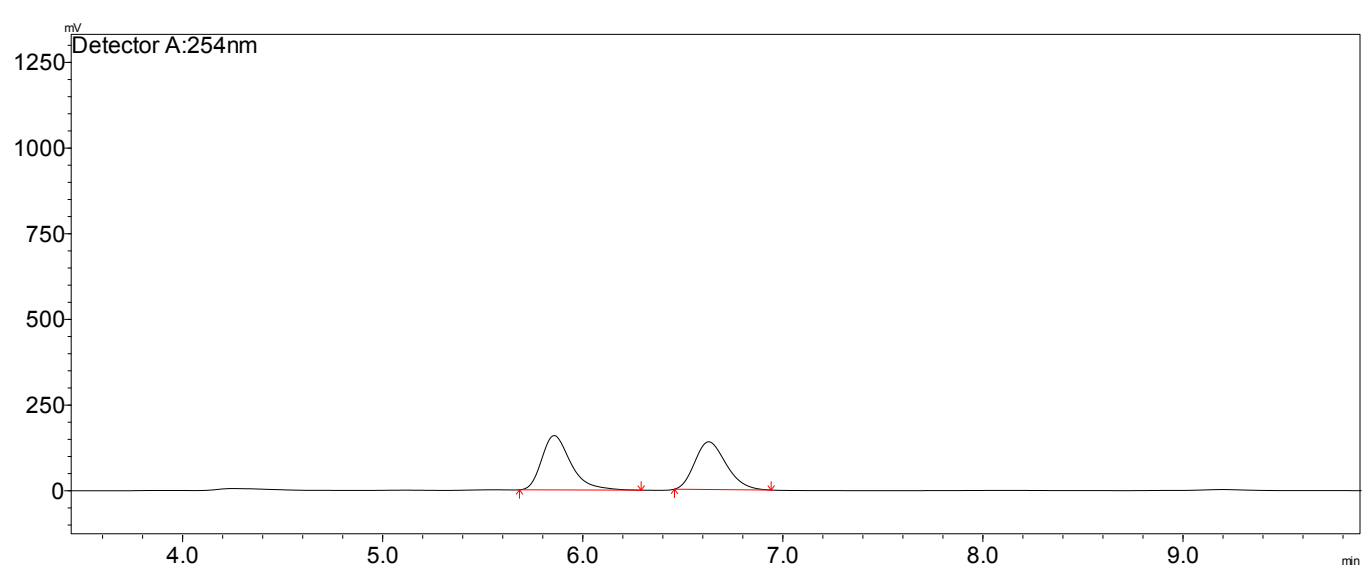

\begin{tabular}{|c|c|c|c|c|c|}
\hline Peak\# & Ret. Time & Area & Height & Area \% & Height \% \\
\hline 1 & 5.853 & 1606629 & 158710 & 51.397 & 53.312 \\
\hline 2 & 6.625 & 1519280 & 138992 & 48.603 & 46.688 \\
\hline Total & & 3125909 & 297701 & 100 & 100 \\
\hline
\end{tabular}

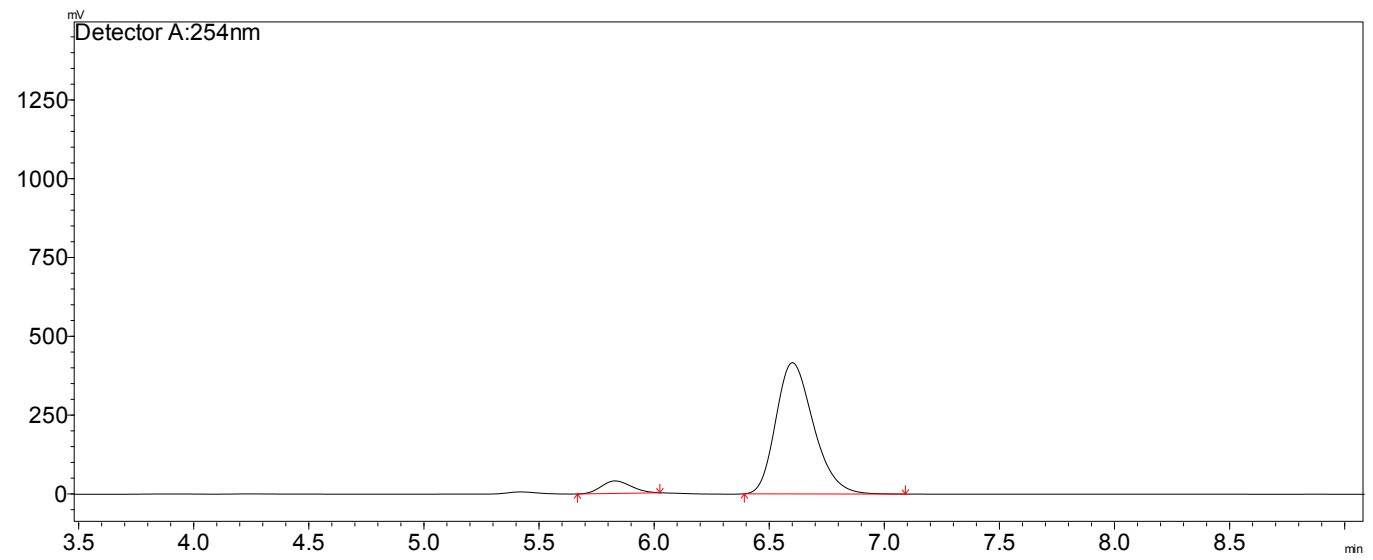

\begin{tabular}{|c|c|c|c|c|c|}
\hline Peak\# & Ret. Time & Area & Height & Area \% & Height \% \\
\hline 1 & 5.827 & 359312 & 39753 & 7.059 & 8.709 \\
\hline 2 & 6.597 & 4730680 & 416703 & 92.941 & 91.291 \\
\hline Total & & 5089991 & 456455 & 100 & 100 \\
\hline
\end{tabular}

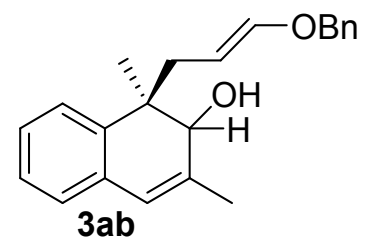



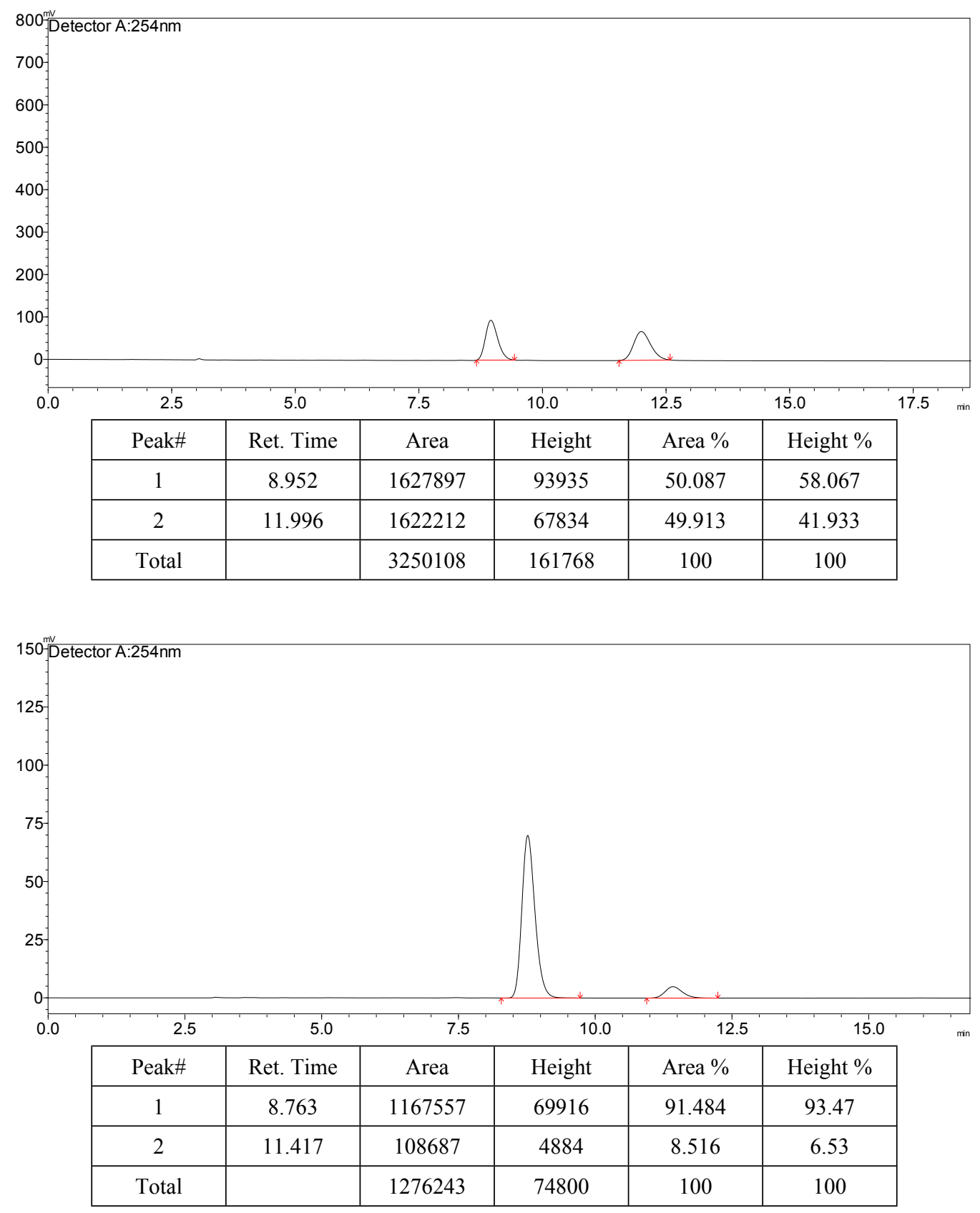